\title{
Broadband for Remote and Rural Communities
}

A project in innovative network technologies and their commercialisation potential

by

\section{Duncan Ewan Cameron}

A thesis submitted in fulfilment of the requirements for the degree of

Master of Innovation and Commercialisation

Wellington School of Business and Government

Victoria University of Wellington

2021 



\begin{abstract}
Wireless Internet Service Provider (WISP) networks are an essential part of providing Internet connectivity in remote and rural areas. Typically operating where large commercial Internet service providers deem it financially unviable to do so, WISPs frequently deal with sparse population densities and financial constraints that inhibit reliable network performance. Venture Networks, a research partner, and a WISP that serves the rural Horowhenua Region, has observed that many WISPs fail to become sustainable businesses, and pondered what could be done to improve outcomes for those providing Internet access where limited other options exist. This research proposes to investigate what commercial opportunities may exist for assisting WISP operators to run their networks in an easier, sustainable manner.
\end{abstract}

To encourage the ubiquitous deployment of high-performance WISP networks, it was recognised that first the unique challenges faced by WISP operators on a wide scale must be understood. A Grounded Theory research methodology was adopted, and a study was conducted to develop an objective understanding of issues commonly encountered by WISP network operators. Interviews were conducted with WISP network operators, digital advocacy organisations, and network engineering consultants. Interview participants came primarily from New Zealand and North America. Of the New Zealand participants, nearly every WISP known to operate within the country was interviewed.

Results of the Grounded Theory study helped to isolate a potential Minimum Viable Product (MVP) and formed the basis for product development and commercialisation. It was shown that start-up and established WISPs frequently lack the technical knowledge to design and implement high-performance networks. Unsurprisingly, a WISP operator that does not thoroughly understand their network will struggle to operate it without difficulty. Consequently, it was proposed that developing a platform around the shortcomings in the knowledge that WISP operators have would be appropriate. Given the proposed platform 
would take form as a physical network appliance, it was decided that initially leveraging a disruptive, solution provider business model would be appropriate. The business model chosen enables Venture Networks to work directly with other WISPs, ideally forming longterm relationships through a customer-centric approach.

Venture Networks has several engineering resources devoted to continued development of the proposed MVP, and envisions developing an ecosystem of products to assist WISPs in operating higher-performance, higher-reliability networks. Ongoing testing of the platform is being carried out with Venture Networks, and a small number of other WISPs selected during the interview phase of this research. Feedback from the trials is continuously integrated into revised versions on the platform. Development of the platform has been carried out using the Agile product development methodology, which enables feedbackresponsive development and quick-to-release iterations of the platform.

Upon final release, it is envisioned that the platform designed will not only enable WISPs to design and implement networks in a straightforward manner, but also offer significant costsavings over alternative solutions available for sale today. For a prospective WISP operator without strong technical competencies, improving Internet connectivity in a remote or rural area would be a daunting challenge. The proposed MVP seeks to make such a task achievable. 


\section{Acknowledgements}

Firstly, I would like to thank my supervisor, Dr Alvin Valera. Without your guidance, I would not be where I am today. I thank you for your support and for enabling life-changing opportunities. Additionally, I would like to thank other members of the Victoria University of Wellington Engineering and Computer Science faculty. Thank you to Professor Winston Seah for giving me the chance to extend my passion for rural broadband connectivity through further research. Thank you also to (soon to be Dr) Matt Stevens. Matt, without your passion for SDN and its applications I would be at a great loss.

I could not have completed this thesis without academic support staff such as Jacquie Harper, and the original programme administrator, Dr Jenny Douché. Thank you both for facilitating such an enjoyable programme to be a part of. Thank you to my fellow Master of Innovation and Commercialisation students - you made the taught component of this programme a time worth remembering.

To Grahame Bennie - thank you for believing in what I am doing and, in the infrastructure that we have already built. It is satisfying to see our work already paying off. Without your financial support, we would not have been able to build what we have. I cannot wait to see what the future holds.

And of course, to my friends and family; thank you all for being there for me. Your support means the world.

Finally, I would like to thank my loving partner, Priyanka. Carrying out this research project was not lonely with you by my side.

\section{Duncan Cameron}

January 2019. 
A society grows great when old men plant trees whose shade they know they shall never sit in Greek proverb 


\section{Contents}

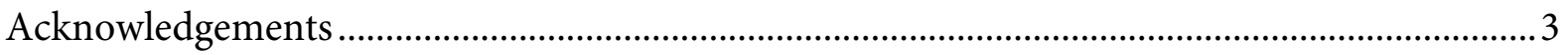

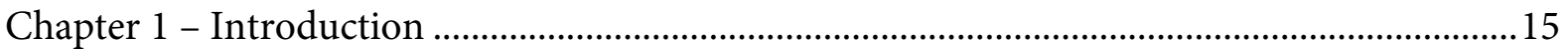

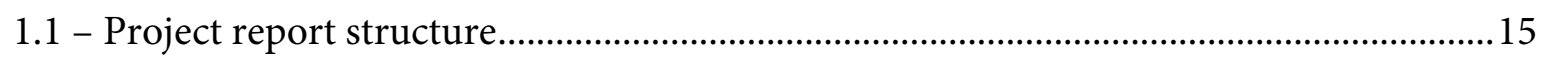

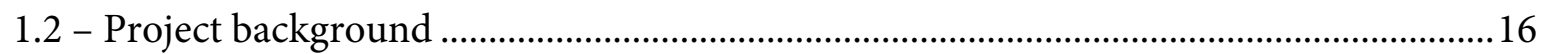

1.3 - Project partner (Venture Networks Limited) ............................................................ 18

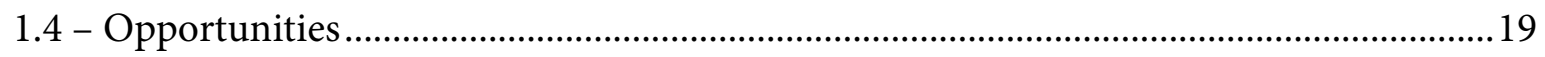

1.5 - Proposed project development and relevant literature.................................................20

1.5.1 - Proposed business model development .................................................................21

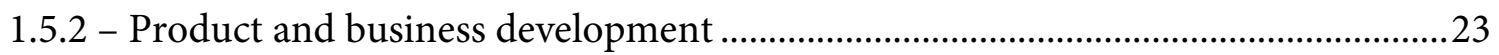

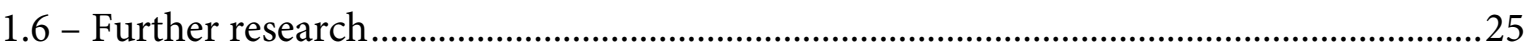

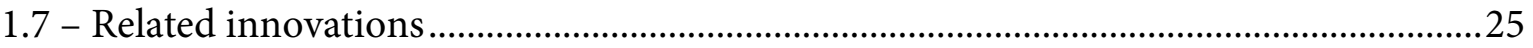

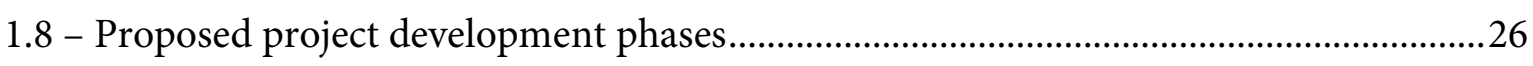

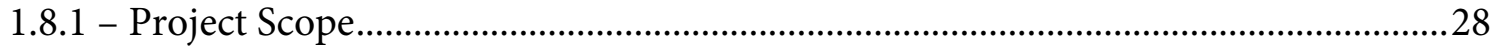

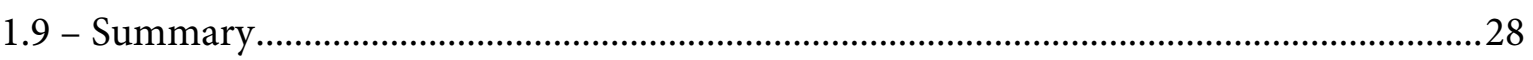

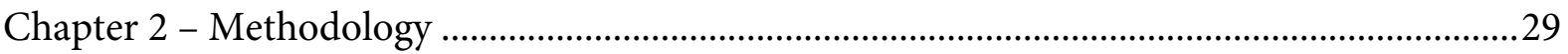

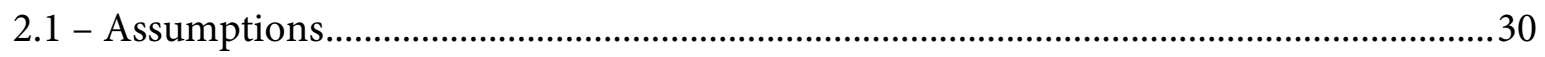

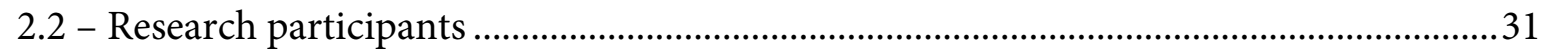

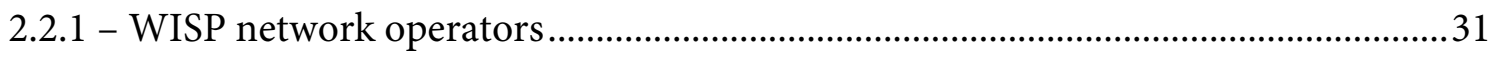

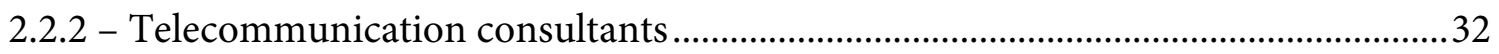

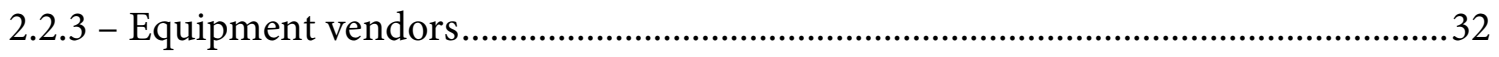

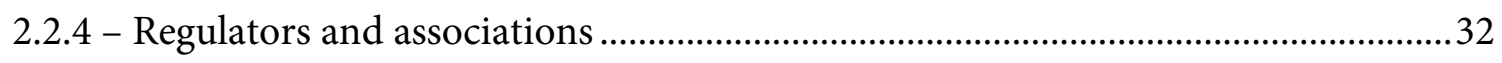

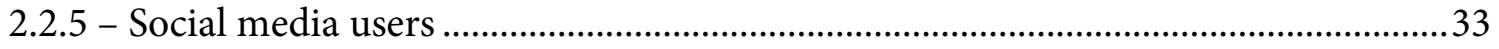




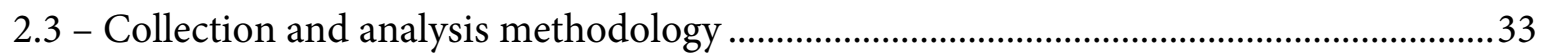

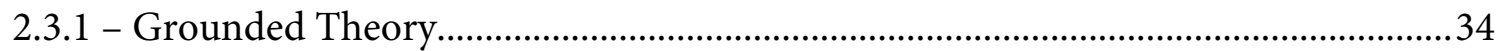

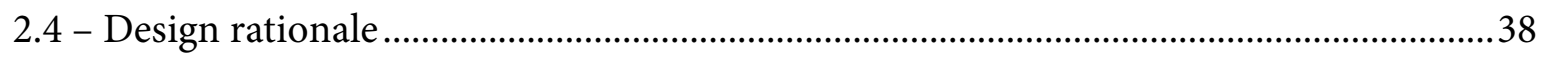

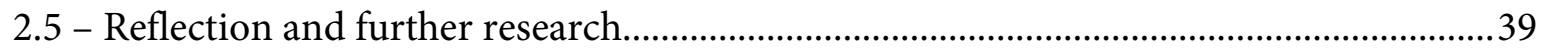

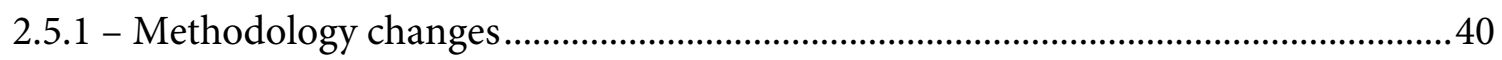

2.6 - Product and business model development methodology.................................................. 41

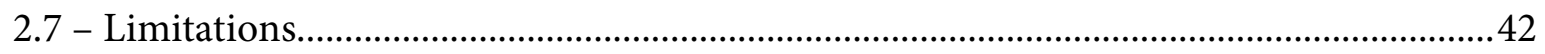

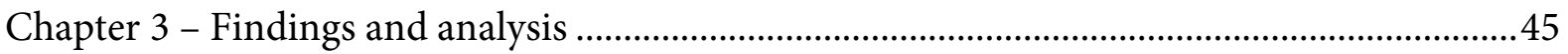

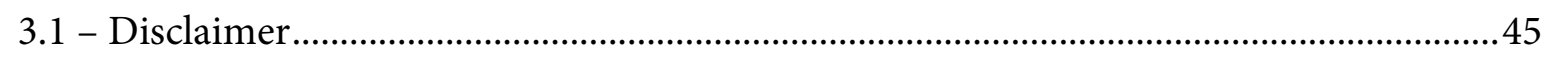

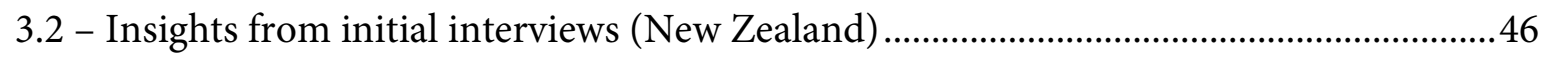

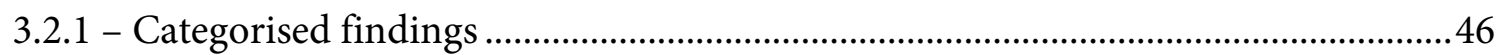

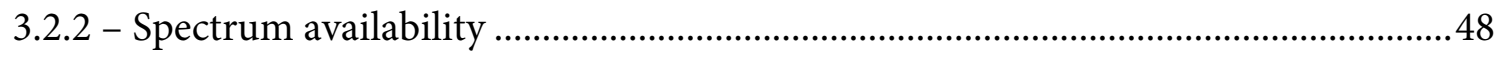

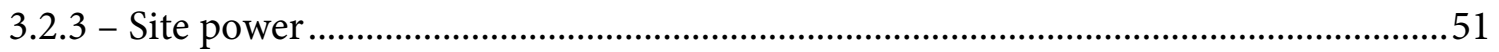

3.2.4 - Capital expenditure .............................................................................................. 51

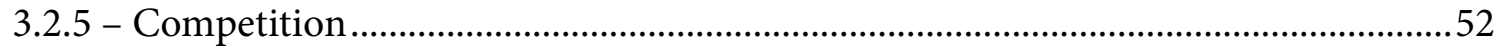

3.2.6 - Grant allocation and the Rural Broadband Initiative ..............................................54

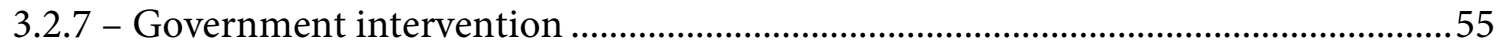

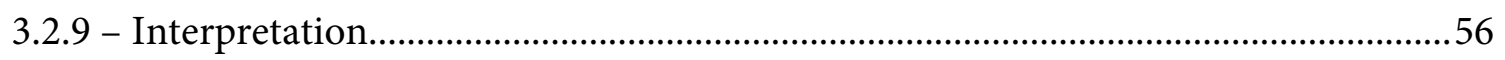

3.3 - Next generation WISP networks: A Grounded Theory.................................................57

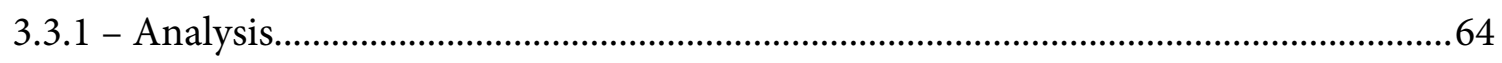

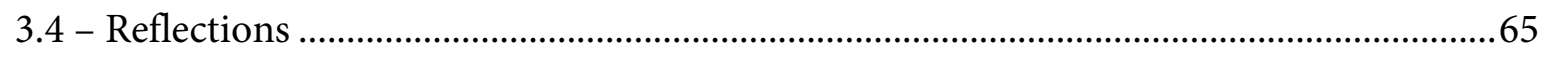

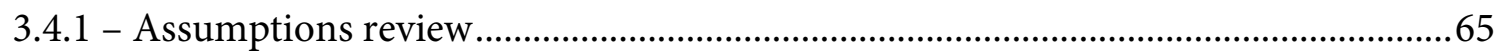

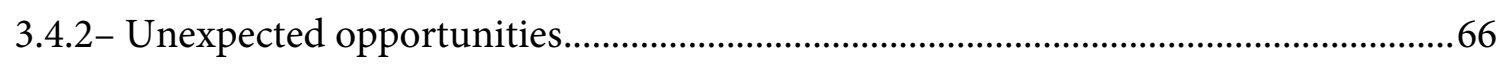




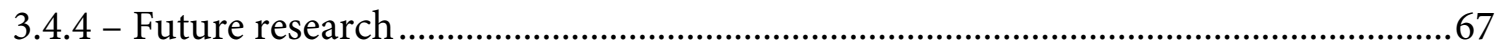

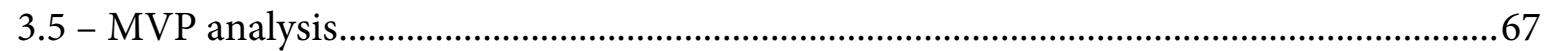

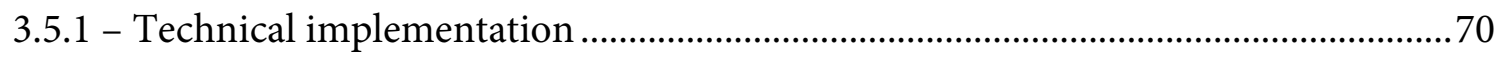

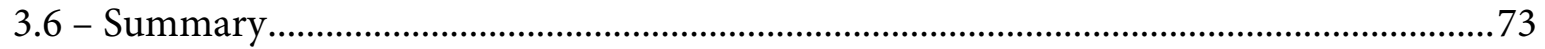

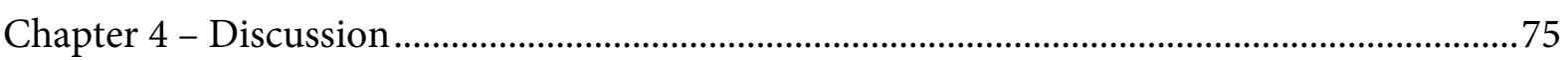

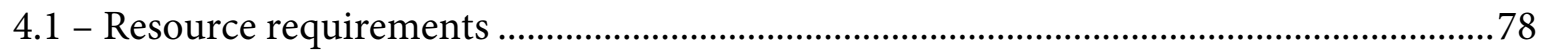

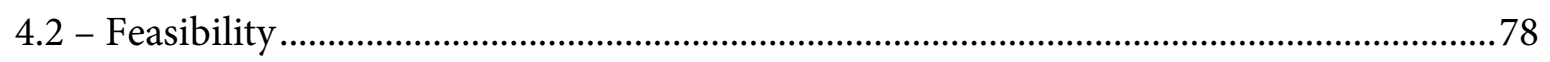

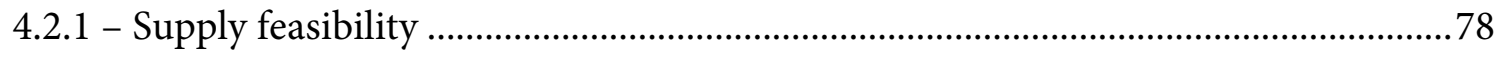

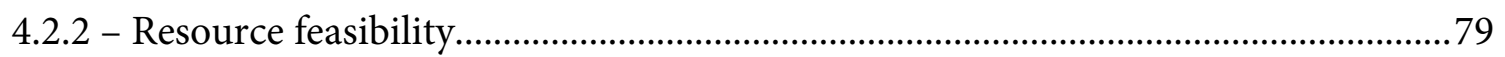

4.2 .3 - Concept feasibility .............................................................................................

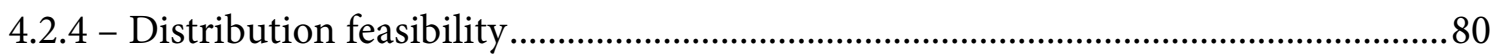

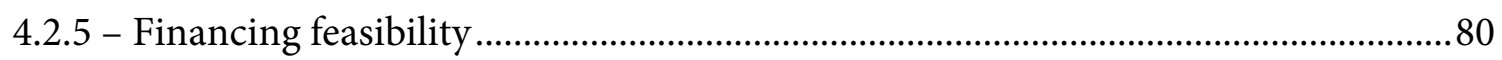

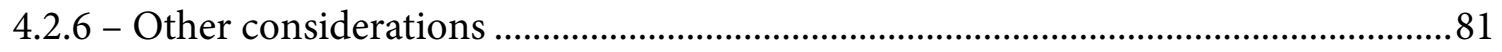

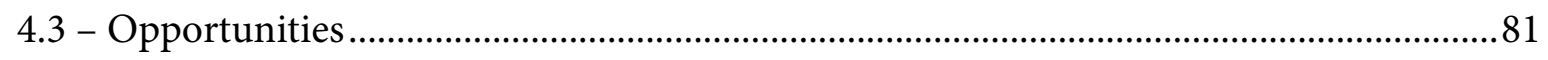

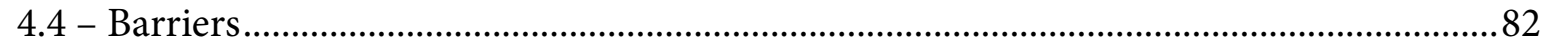

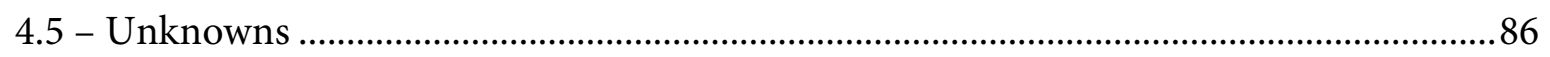

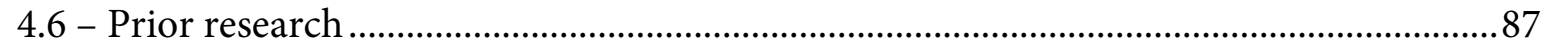

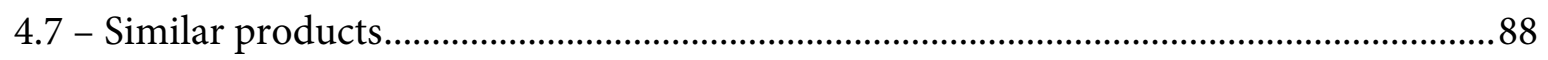

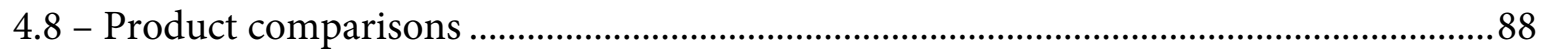

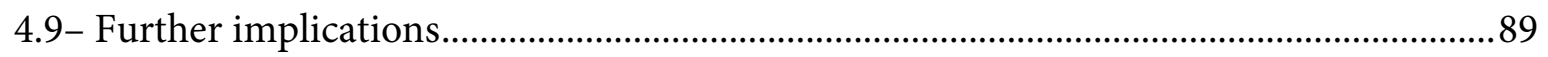

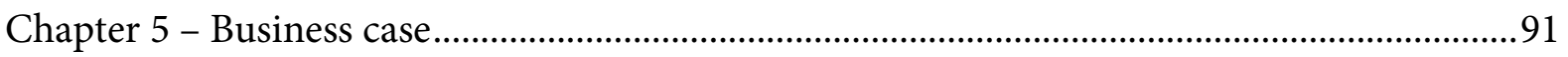

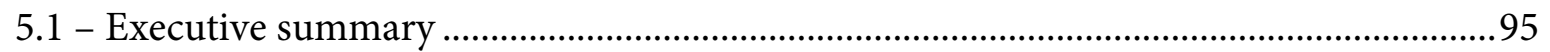

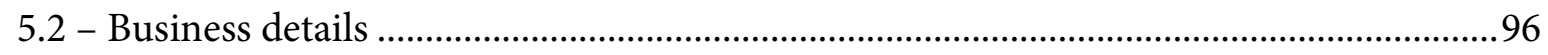




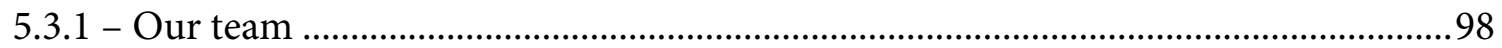

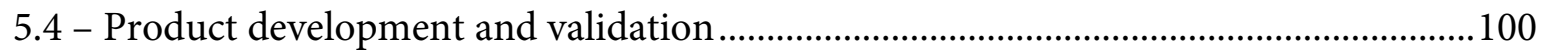

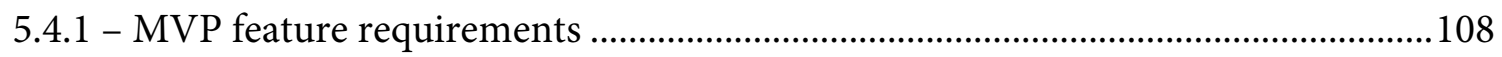

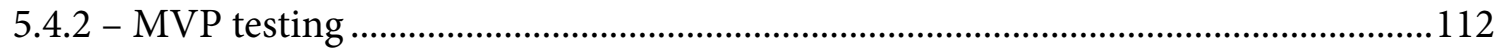

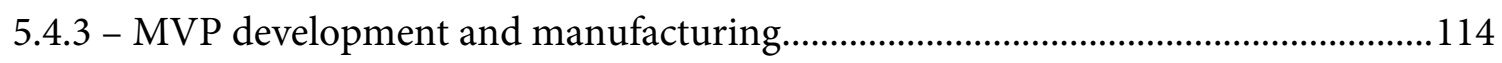

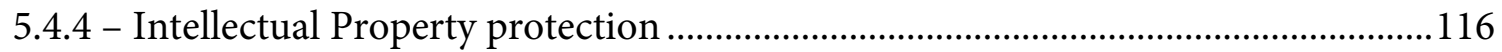

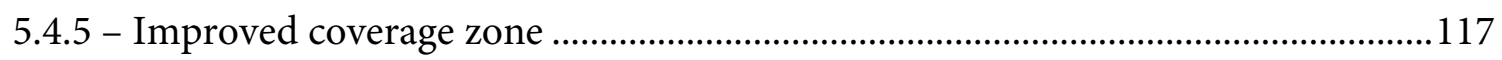

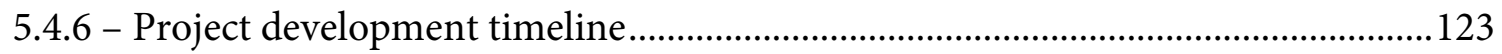

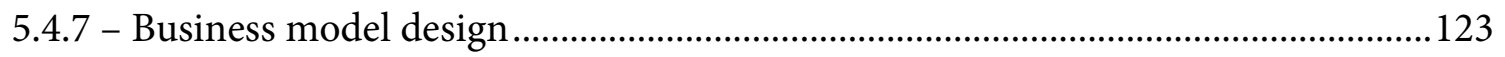

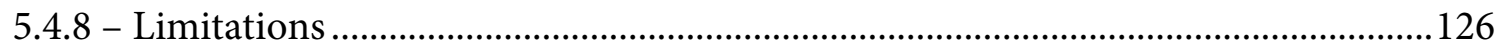

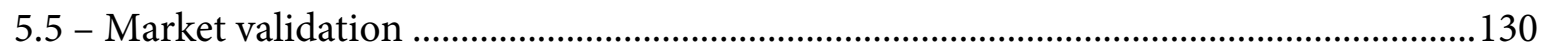

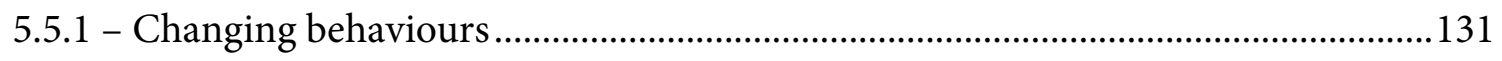

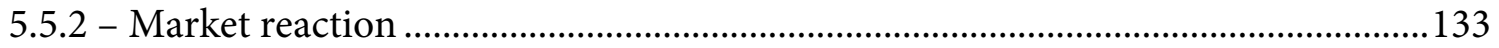

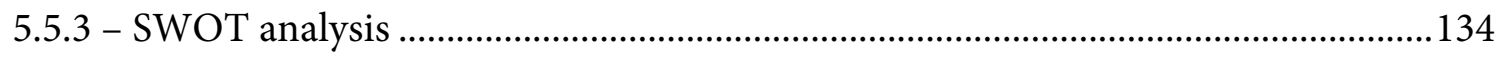

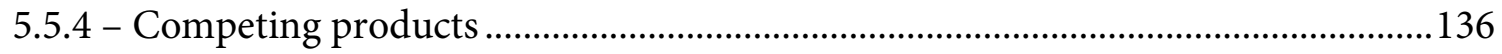

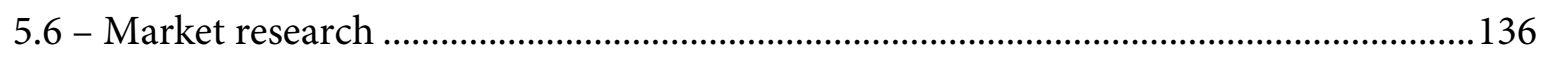

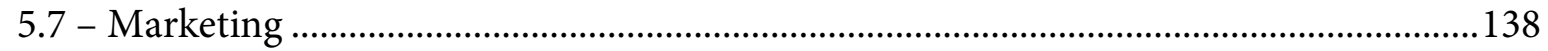

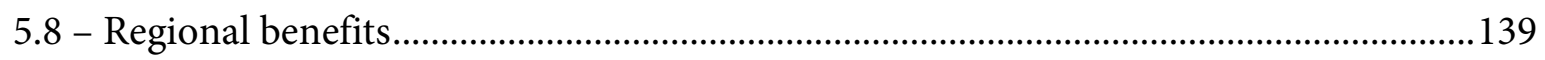

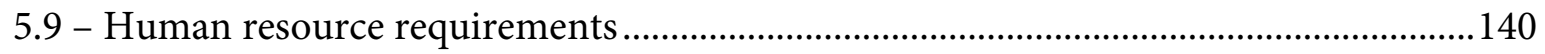

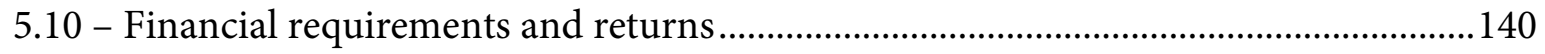

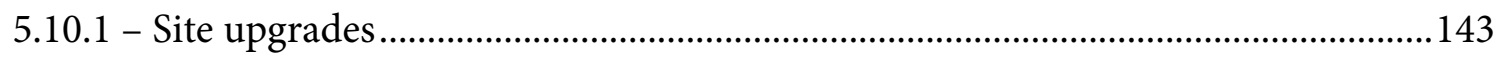

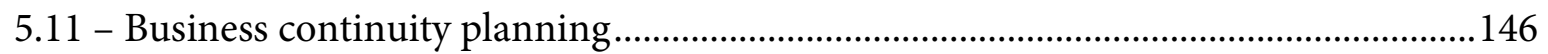

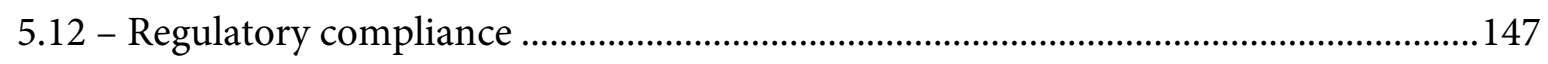


Chapter 6 - Conclusion

References.

Appendices 


\section{Figures}

Figure 1 - Iterative development process. ............................................................................. 40

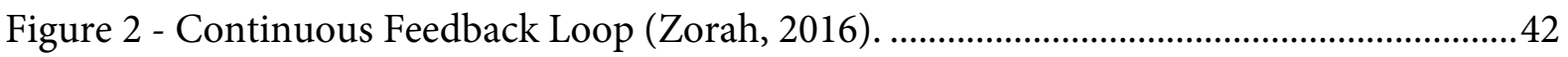

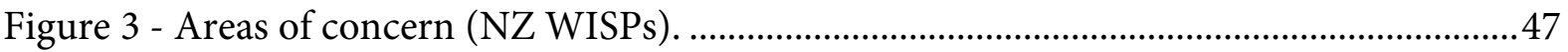

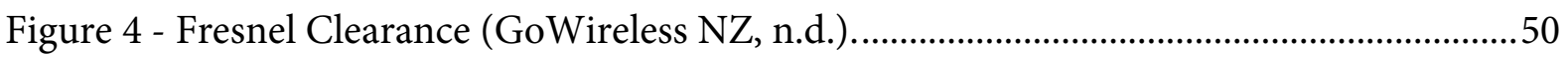

Figure 5 - Wisp Talk and Wisp Talk (Newbies and Startups) comparison diagram.................60

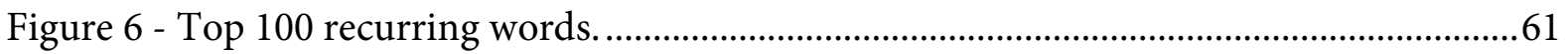

Figure 7 - Top 10 recurring words. .........................................................................................62

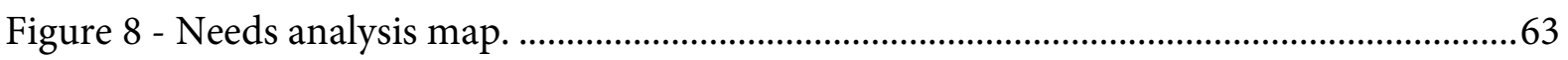

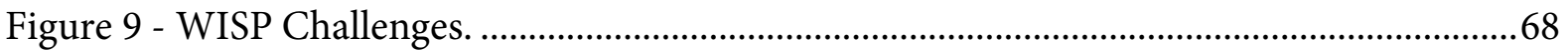

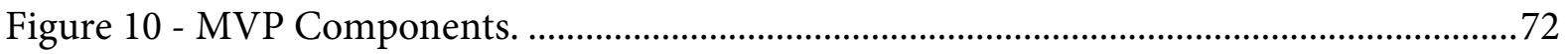

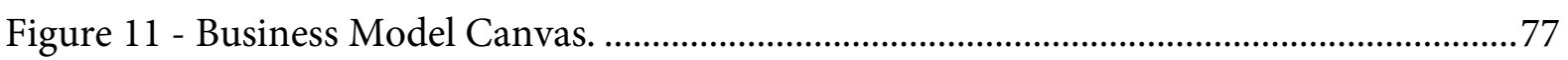

Figure 12 - Broadcast traffic in a bridged WISP network (Discher, 2013). ................................83

Figure 13 - Broadcast traffic in a routed WISP network (Discher, 2013)................................84

Figure 14 - Areas of concern (New Zealand WISPs)....................................................................104

Figure 15 - Wisp Talk and Wisp Talk (Newbies and Start-ups) comparison diagram...........107

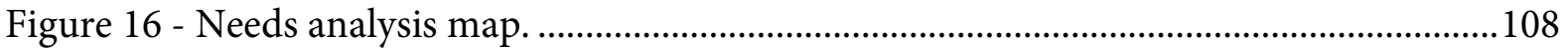

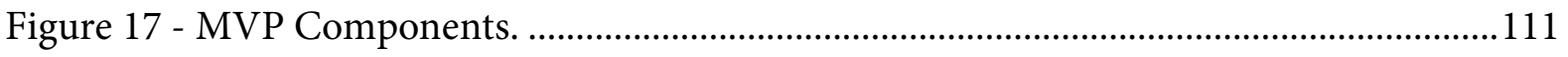

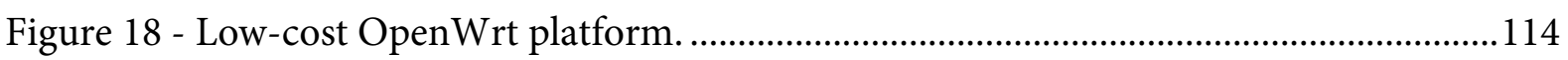

Figure 19 - Venture Networks propagation map......................................................................118

Figure 20 - Internet access at Motuiti and Paranui Marae............................................................119

Figure 21 - Venture Networks expanded network propagation map......................................120

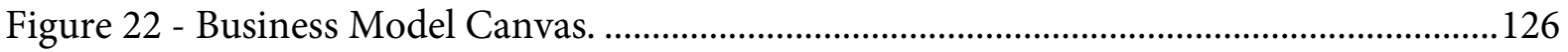

Figure 23 - Spanning Tree Protocol (Coxhead, 2013) ...............................................................128

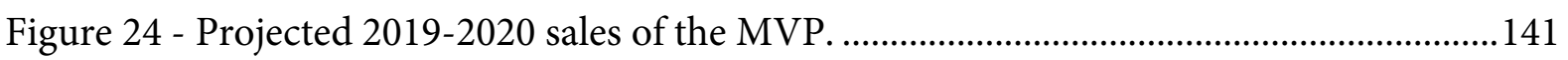

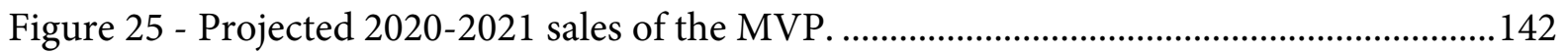

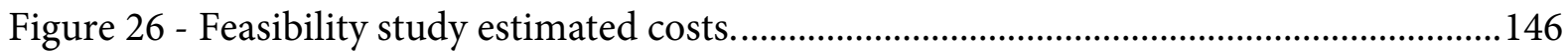




\section{Glossary}

CPE - Client Premises Equipment. The radio and router equipment installed by a WISP at a customer location.

CPU - Central Processing Unit.

dBm - Decibel-milliwatts. A measure of power in decibels, with reference to 1 milliwatt. DFS - Dynamic Frequency Selection. A technology used for radar detection that enables sharing of the $5 \mathrm{GHz}$ spectrum where licensed use must be protected, i.e., weather radar operating within the $5 \mathrm{GHz}$ spectrum.

EIRP - Effective/Equivalent Isotopically Radiated Power. The EIRP of a radio transmitter is the product of the TX, or transmit power, and the gain of the attached antenna.

IEEE - Institute of Electrical and Electronics Engineers. A professional association that is also responsible for academic publishing and the development of standards.

MVP - Minimum Viable Product. The prototype product designed with the necessary features to satisfy the demand of the initial target customers.

OpenFlow - A communications protocol enabling the control of a device's forwarding plane.

OpenMul - A high-performance OpenFlow 'SDN' controller.

OPEX - Operational Expenses. Ongoing costs of running a business.

OSPF - Open Shortest Path First (routing protocol).

PoE - Power over Ethernet. A method of powering network equipment on a network through the same Ethernet cable data is sent over.

PoP - Point of Presence.

RBI1/RBI2 - Rural Broadband Initiative. The New Zealand Government programmes to bring better rural Internet connectivity to more New Zealanders.

RSTP - Rapid Spanning Tree Protocol. Enables layer-2 loop avoidance on networks, with faster convergence times than STP. 
SDN - Software-Defined Networking. An architecture that typically decouples the forwarding and data-planes of a network to enable greater programmability and control. Also commonly used to refer to OpenFlow and OpenFlow enabled controllers such as OpenMul. STP - Spanning Tree Protocol. Enables layer-2 loop avoidance on networks. UFB - Ultra-Fast Broadband. The New Zealand Government scheme to bring fibre optic broadband to New Zealand homes, schools, and businesses.

WISP - Wireless Internet Service Provider. Typically, small companies that provide Internet connectivity to remote and rural communities. 


\section{Chapter 1 - Introduction}

An analysis of Wireless Internet Service Providers (WISPs) was conducted for this research. Objective, data-driven product development and market validation was carried out, and a potential Minimum Viable Product, or MVP was designed based on the findings. The proposed MVP designed leverages Software-Defined Networking (SDN) and provides prospective WISPs with a low barrier to entry platform to build performant, robust fixedwireless broadband networks. It is anticipated that the proposed MVP will additionally reduce network operational expenses through the reduced need for technically skilled staff. A combination of qualitative and quantitative methods were employed in the data-driven design of the MVP, with Grounded Theory being selected as the primary research methodology.

This project report has been written following the completion of the taught components of the Master of Innovation and Commercialisation programme at Victoria University of Wellington. Product and market validation throughout this research has been completed in conjunction with a project partner, Venture Networks Limited, a WISP based out of Levin in the lower North Island. Venture Networks has also been responsible for a significant portion of product design funding for this research.

\section{1 - Project report structure}

The structure of a Master of Innovation and Commercialisation project report differs from that of a traditional thesis. Sections are briefly detailed below:

\section{Chapter 1 - Introduction}

This chapter introduces the background of the research, the research partner, and potential business models. Additionally, the scope of the research, related innovations, and the opportunities for developing an innovative product specifically for WISP network operators are covered. 


\section{Chapter 2 - Methodology}

The second chapter informs the reader of how the research was designed and why it was done this way.

\section{Chapter 3 - Findings and analysis}

Following the methodology, the third chapter presents the results of data collection and their implications to the project.

\section{Chapter 4 - Discussion}

The fourth chapter discusses significant findings and what they mean for the project.

\section{Chapter 5 - Business case}

The fifth chapter is the most significant. Findings and recommendations are consolidated into a business plan designed for the research partner and potential investors. Emphasis is placed on the business case chapter, as it is the core component of the entire research.

\section{Chapter 6 - Conclusion}

The final chapter provides a closing overview of the project, decisions that were made, their implications, and what further research might be carried out.

\section{2 - Project background}

Growing up in the rural Horowhenua meant that fast and reliable Internet access was something to be dreamed of. Dial-up Internet access was only replaced in much of the region with (slow by modern standards) ADSL in 2011. At present, ADSL is still the fastest option in the area, excluding wireless services provided by the research partner, Venture Networks. Knowing that slow or no connectivity is a barrier to education, leisure, work, and much more, Venture Networks was founded in 2013 and set out to make a difference for the local 
community. Several years on, Venture Networks offers high-speed wireless connectivity at affordable prices across much of the rural Horowhenua district.

However, future ambitions do not stop there. Other communities around New Zealand and the world suffer from slow, or non-existent Internet connectivity. Unfortunately, there is no standardised approach to building Internet infrastructure in remote and rural communities. If an entrepreneur interested in improving connectivity outcomes in any given rural area were to ask a panel of experts for advice, it is likely many would have their own, belligerent, perspectives regarding development. It has been observed by the researcher that some such entrepreneurs often lack a strong background in networking, which makes implementing, maintaining, and scaling fast and reliable rural broadband networks a challenging task, even with the assistance of experienced consultants. Lacking expertise is especially problematic when network faults occur, and someone without strong networking competencies is not readily available for assistance.

For WISPs to run their networks effectively, the fundamental problem at hand must be solved, i.e., strong technical competencies are inherently required for operating and maintaining an Internet-centric business. Networking equipment available to WISPs has had drastic improvements in certain areas, namely wireless radios, thanks to innovative engineering from companies such as Ubiquiti Networks. The barrier to entry for building fixed wireless links was drastically lowered with Ubiquiti's entry to the telecommunications market, which swiftly prompted some incumbent telecommunications vendors to follow suit and improve usability of their products. Networking vendors have to date, however, failed to innovate the usability of high-speed, high-reliability routing and switching platforms for those without strong technical competencies.

A Grounded Theory methodology was employed to assess what key challenges are commonly faced by WISPs. This approach enabled a practical means of product development to take place. Following the collection and analysis of data, it became evident that building an 
innovative solution using the routing protocols commonly used on WISP networks would not achieve anything innovative or useful to those who need it the most. Emphasis was placed on how to improve the usability, performance, and ease-of-deployment of network forwarding devices that are typically used in WISP points-of-presence. The proposed MVP was later designed specifically for the WISP market to use, following a low barrier to entry approach.

The OpenFlow protocol was selected for use on the proposed MVP. Essentially, OpenFlow is a protocol that enables Software-Defined Networking (SDN). OpenFlow enables network programmability, separating control of the network from a router/switch and moving it to a separate, often central controller. In the case of this project, network control has been achieved using a highly efficient controller written in the $\mathrm{C}$ programming language, with an easy-to-use interface planned for end-users. The OpenFlow protocol itself will eventually be replaced by another application known as Stratum, which is currently being developed by the Open Networking Foundation. It is unknown when Stratum will release, but it is estimated it will be sometime in early 2019. Because of this, hardware for the research was selected carefully to give it the best chance of working with the next-generation Stratum platform upon release.

\section{3 - Project partner (Venture Networks Limited)}

As mentioned, this research has been carried out in conjunction with Venture Networks. To reiterate, Venture Networks was formed in 2013 out of the need for better Internet infrastructure in the rural Horowhenua district. Working as a Director of Venture Networks has been influential in the development of this research. Being able to observe the day-to-day operations has provided insights into recurring operational problems, many of which have been tested and proven to be frequently problematic to other providers too, often on a much wider scale. As well as providing good starting points for research, Venture Networks' 
wireless network has also proven to be useful as a testbed for experimental technology utilised throughout this research.

\section{4 - Opportunities}

In its early stages, this research could have been steered towards solving any number of issues faced by WISPs. However, it was understood early on that substantial market opportunity comes from prospective WISPs who are in the process of initial network construction. There is much enthusiasm from technical and non-technical entrepreneurs to build infrastructure in underserved areas. For this research, capturing these entrepreneurs' innovative spirit is critical, as is informing existing network providers of the proposed platform and its benefits. Additionally, the proposed platform will be the first of its kind commercially available. Because of these considerations, for the proposed platform to have the best chance of success, an emphasis was placed on the ability to:

- Exploit the non-technical background of interested entrepreneurs in creating highspeed, high-reliability, scalable networks.

- Persuade existing network operators (especially those with performance and scalability issues) of the value in switching to the proposed platform.

The proposed platform will solve network performance and scalability issues for WISP networks, while simultaneously reducing the need for on-hand technical staff. The zero-touch approach will enable any network provider to automate what would otherwise be complicated traffic-engineering functionalities. Automating traffic engineering enables service providers to achieve with minimal effort what would otherwise potentially require years of network engineering experience to implement. As it stands, to achieve what the proposed platform will accomplish, the network operator must use an assortment of networking protocols. Existing service providers typically use a variety of routing protocols to achieve better network reliability and control. These are typically a combination of OSPF, MPLS, and BGP. 
To an entrepreneur with a limited networking background, experienced consultants using unfamiliar technical terms is meaningless. What is even more problematic is what happens in a situation where an entrepreneur, including one with a limited networking background, implements a complicated solution they do not fully understand. On several occasions, network operators were observed publicly expressing a need for urgent help as something set up by a consultant on their network had stopped working, preventing their clients from being able to access the Internet. Much like how a car owner does not need to understand the complex inner workings of an automatic gearbox, a WISP operator does not need to understand the inner workings of the proposed platform to benefit from better reliability, performance, and scalability.

\section{5 - Proposed project development and relevant literature}

The primary objective of this research is to discover and exploit the commercial opportunities for developing an innovative network product that can enable ubiquitous remote and rural Internet development. To help lower the barrier to entry for building and scaling WISPs, understanding what points of difference market leaders such as Ubiquiti Networks and Netonix have are beneficial. Additionally, to better understand existing barriers to such infrastructure development, and what must be done to overcome them, literature related to network sustainability is analysed.

It is important to note that little academic literature has been focussed on approaches making remote and rural broadband networks sustainable. Most literature focusses heavily on measuring and understanding the usage-patterns of rural broadband adoption, with a few key exceptions. A lack of literature from a wide range of academic sources suggests more research needs to be carried out in this area. One may naively believe this is due to there being a limited opportunity for research in this area; however, an estimated $52 \%$ of the global population is still without Internet access, as of 2017, with Internet penetration being as low 
as $21 \%$ throughout Africa (Blantz \& Summer, 2011; International Telecommunication Union, 2017).

\subsection{1 - Proposed business model development}

Former Apple engineer Robert Pera started Ubiquiti Networks in 2005. After working on testing wireless devices at Apple, Pera realised there was potential for similar wireless devices to be used over long distances, and set about creating Ubiquiti Networks (Raza, 2017). Taking an innovative approach to business model design, Ubiquiti has now achieved market penetration in adjacent telecommunications markets, and has grown to over USD 800 million in annual revenue. Surprisingly, Ubiquiti's growth has been accomplished all while having no salesforce (Ubiquiti Networks, 2017). Ubiquiti has achieved these advances through an innovative business model, as summarised below:

1. Targeting underserved markets (WISPs and later enterprise customers).

2. Developing innovative, high-performance telecommunications products.

3. Low-cost operations.
a. No salesforce.
b. Hiring elite engineers from low-cost countries (China and Eastern Europe).
c. Contract manufacturing in China.

4. Disruptive pricing.

Another company with a similar business model to that of Ubiquiti is Netonix. The researcher has observed that many WISPs have started using Netonix for their PoE enabled network switches, as there are limited innovative alternatives to them currently on the market. Netonix was founded in 2014, but unlike Ubiquiti, manufactures most of its equipment in North America (Netonix, 2014). Netonix is a private company, and its sales data is not publicly available. However, despite the higher price of their products, Netonix has gained a strong position within the WISP market. The market position and positive 
reputation Netonix has gained is likely due to their superior product and their emphasis on North American manufacturing.

Ubiquiti and Netonix both provided insights into what business model might be appropriate for the proposed platform. Business model prototyping was reiteratively completed as business requirements evolved throughout the research project. Business model prototyping itself was carried out using the Strategyzer Business Model Canvas. Use of the Business Model Canvas has gained credible support from academics and businesspeople around the world (Chesbrough, 2010; Eppler, Hoffmann, \& Bresciani, 2011). Compared to alternative approaches, the Business Model Canvas, by design, enables a universally recognised idea of what a business model is to be established (Osterwalder, 2004; Stampfl, 2016). The Business Model Canvas was also used in conjunction with the Strategyzer Value Proposition Canvas. Much like its counterpart, the Value Proposition Canvas provides a rapid means of prototyping what value an MVP may have.

Leveraging a disruptive business model for the proposed platform also depends on understanding what value prospective products might have in the WISP market. Research from Blantz and Summer (2011), Ben-David (2015), and Nungu, Olsson, and Pehrson (2012) recognise the necessity for low-cost, low-power and low barrier to entry equipment that is robust enough to stand up to a wide range of electrical and environmental conditions. Hasan, Ben-David, Bittman, and Raghavan (2015) argue that Software-Defined Networking can be leveraged to alleviate the technical skills required for sustainably operating remote and rural broadband networks. Unfortunately, little other topical academic research outside of the sources identified from the KTH Royal Institute of Technology in Sweden, and from the University of California, Berkeley, exists.

Additional academic sources may have helped to identify unforeseen gaps in underserved rural markets, ultimately influencing the design of the proposed platform and the choices made during business model development. Due to limited published research, the 
observations, interviews, and some quantitative data gathered from WISPs functioned as an essential part of the design and development of the business model throughout this research. It is hoped that following the long-term development and real-world testing of the proposed MVP designed, additional research will be carried out and published on remote and rural broadband network sustainability.

\subsection{2 - Product and business development}

Golder and Tellis (1993) show that first movers, or those to first introduce a new product to market, do not necessarily have an advantage over their competition, with a staggering $47 \%$ failure rate. Examples are not difficult to come by; Facebook and Google are two prominent examples of successful companies that were not the first to market, but rather fast followers. Essentially, fast followers build upon what has already been completed by others. Before Facebook, there was Friendster and Myspace. Facebook entered the social media market after both companies but grew explosively, dominating the market. In comparison to the first movers, fast followers studied had a much lower, $8 \%$ failure rate. Minimum Viable Product (MVP) development was carried out with these figures in mind.

An appropriate product development methodology was selected for the technical development of the proposed platform, or MVP. Several product development methodologies exist, including Agile, Waterfall, Rapid Application Development, Rational Unified Process, and so on (Goodman \& Henry, 2010). The table below briefly shows strengths and weaknesses for both methodologies deliberated for use in the context of the proposed MVP:

\begin{tabular}{|c|c|c|}
\hline Methodology & Strengths & Weaknesses \\
\hline Waterfall & 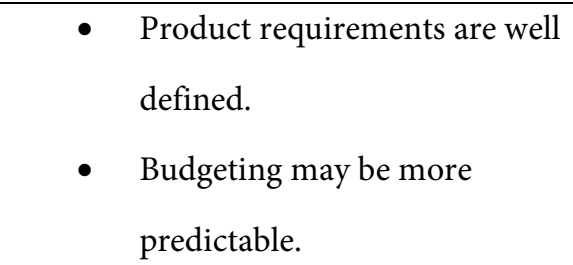 & $\begin{array}{l}\text { - Less interaction and collaboration } \\
\text { with customers. } \\
\text { - Lacks development flexibility. }\end{array}$ \\
\hline Agile & $\begin{array}{l}\text { Rapid product feature } \\
\text { addition/experience }\end{array}$ & $\begin{array}{l}\text { - May initially lack end-user } \\
\text { documentation. }\end{array}$ \\
\hline
\end{tabular}




\begin{tabular}{|l|l|l|}
\hline & improvement through user & Re-working code/product may be \\
interaction (forum, social & required due to quick development \\
media, various others). & turnover. \\
$\bullet$ & Potentially faster time-to- & Lack of certainty over when \\
& market. & projects will be complete. \\
& Potentially higher productivity.
\end{tabular}

Table 1 - Development methodologies. Adapted from Goodman and Henry (2010).

Due to its flexibility, an Agile approach to product development was selected. Some uncertainty had to be taken into consideration to build the best MVP possible, in collaboration with other WISPs, industry stakeholders, and academics. Dealing with development uncertainty and differing opinions meant the more-linear Waterfall approach was not suitable. It was acknowledged that a base product should be defined, and that additional features would be designed through collaborative input, making an Agile approach ideal. The Agile product development methodology itself was initially designed for software development (ManagementNext, 2012). Since its inception in 2001, the Agile approach has been applied broadly outside of software development and even used as a marketing tool by salespeople (Varma, 2015). This research recognises that an Agile approach in true form should adhere to the Agile Manifesto, and value:

\section{Individuals and interactions over processes and tools}

Working software over comprehensive documentation

Customer collaboration over contract negotiation

Responding to change over following a plan

(Beck et al., 2001).

Michael Porter's four generic competitive strategies were also considered. These strategies include cost leadership, market differentiation, innovation differentiation, and market focus. Porter argued that for companies to be successful, they should adopt and adhere to at least one strategy (Porter, 1998). In the case of the proposed MVP, market differentiation and 
innovation differentiation are both applicable. The market targeted is underserved, as is shown by global Internet penetration statistics. The innovation proposed can also be differentiated, as the proposed MVP has features unique to it. Strategy recommendations are implicitly covered later in Chapter 5 - Business case.

The proposed use of an Agile methodology and the use of either a market differentiation or innovation differentiation competitive strategy builds an approach like that taken by Ubiquiti Networks towards product development. It is proposed that the MVP will be marketed through working directly with remote and rural network operators, and later through equipment suppliers if early trials are a success, tracking the Ubiquiti approach. During the reiterative data collection stage, and a testament to this approach, several WISPs have already expressed interest in trialling the MVP. Much like Ubiquiti and Netonix, an online forum for support and research and design feedback is also proposed for the MVP.

\section{6 - Further research}

Further research needs to be carried out to find sustainable ways of providing Internet access in remote and rural communities. Undoubtedly, this is a challenge with difficulty that cannot be understated, especially in areas where income is low, and little business case for building potentially expensive Internet infrastructure exists. It was also identified throughout the study that the price of power systems for remote sites (such as solar panels, wind turbines, battery systems) is often the limiting factor to further expansion. Due to this, cheaper development of off-grid power systems, and the development of lower power network equipment (such as routers and radios) would enable further, widespread deployment.

\section{7 - Related innovations}

The proposed MVP uses the OpenFlow protocol, which has enabled network innovation throughout numerous companies, including Internet heavyweight Google, where 100\% of Google's most extensive production datacentre network runs on OpenFlow (Hoelzle, 2012). 
In the case of remote and rural broadband connectivity, OpenFlow has seen no implementation. The application of SDN and OpenFlow for WISP networks was proposed by Hasan, Ben-David, Scott, Brewer, and Shenker (2013), and later investigated in depth by Hasan et al. (2015) and Ben-David (2015). While the applications of SDN within remote and rural broadband networks were found to be beneficial, no SDN-based forwarding platform was developed, leaving opportunity for further research to be carried out. It should also be noted that Ubiquiti uses its own proprietary implementation of SDN for their enterprise UniFi range of products, utilising central management to enable seamless network configuration. However, the UniFi SDN platform is not applicable or related to their service provider range of products.

\section{8 - Proposed project development phases}

The table summarises project objectives, assumptions, opportunities, and unknowns, as well as where each category corresponds to an appropriate development phase.

\begin{tabular}{|c|c|c|c|c|}
\hline $\begin{array}{l}\text { Development } \\
\text { Phase }\end{array}$ & Objectives & Assumptions & Opportunities & Unknowns \\
\hline $\begin{array}{l}1-\text { Market } \\
\text { exploration and } \\
\text { validation }\end{array}$ & $\begin{array}{l}\text { Use Grounded Theory } \\
\text { to understand market } \\
\text { gaps/opportunities. }\end{array}$ & $\begin{array}{l}\text { Many new and } \\
\text { growing remote and } \\
\text { rural broadband } \\
\text { providers struggle } \\
\text { to scale their } \\
\text { networks, build } \\
\text { robust } \\
\text { infrastructure. } \\
\text { Low-budgets, } \\
\text { regional financial } \\
\text { constraints are a } \\
\text { crucial issue for } \\
\text { ubiquitous } \\
\text { broadband access. }\end{array}$ & $\begin{array}{l}\text { Potential } \\
\text { development of } \\
\text { innovative } \\
\text { networking } \\
\text { equipment } \\
\text { (MVP). } \\
\text { Building } \\
\text { infrastructure } \\
\text { solutions that } \\
\text { anyone can } \\
\text { maintain. }\end{array}$ & $\begin{array}{l}\text { Understanding } \\
\text { how those with } \\
\text { limited technical } \\
\text { knowledge can } \\
\text { approach building } \\
\text { telecommunication } \\
\text { networks. } \\
\text { What happens } \\
\text { when third parties } \\
\text { build } \\
\text { infrastructure, and } \\
\text { local communities } \\
\text { cannot maintain it. }\end{array}$ \\
\hline
\end{tabular}




\begin{tabular}{|c|c|c|c|c|}
\hline $\begin{array}{l}2 \text { - Product } \\
\text { development } \\
\text { and validation }\end{array}$ & $\begin{array}{l}\text { Examine gathered } \\
\text { data, construct an idea } \\
\text { of what solution will } \\
\text { have the most impact. } \\
\text { Determine the value } \\
\text { proposition of the } \\
\text { theoretical MVP. } \\
\text { Approach industry } \\
\text { stakeholders with the } \\
\text { proposed platform, } \\
\text { adapt, as necessary. }\end{array}$ & $\begin{array}{l}\text { Industry } \\
\text { stakeholders will be } \\
\text { open to trying new } \\
\text { technology. } \\
\text { Well established, } \\
\text { large service } \\
\text { providers may } \\
\text { struggle to see the } \\
\text { value proposition of } \\
\text { lower barrier to } \\
\text { entry products. }\end{array}$ & $\begin{array}{l}\text { Agile MVP } \\
\text { development. }\end{array}$ & $\begin{array}{l}\text { How to find WISP } \\
\text { operators most in } \\
\text { need of lower } \\
\text { barrier to entry } \\
\text { products. }\end{array}$ \\
\hline $\begin{array}{l}\text { 3- Business } \\
\text { model } \\
\text { development }\end{array}$ & $\begin{array}{l}\text { Identify key industry } \\
\text { stakeholders. } \\
\text { Identify sales channels, } \\
\text { national and } \\
\text { international } \\
\text { distributors. } \\
\text { Investigate global } \\
\text { product supply and } \\
\text { shipping. }\end{array}$ & $\begin{array}{l}\text { Convincing other } \\
\text { service providers to } \\
\text { try any proposed } \\
\text { MVP will be critical } \\
\text { to success. } \\
\text { MVP prototypes } \\
\text { will be developed } \\
\text { and tested before } \\
\text { the business case is } \\
\text { finished being } \\
\text { developed. }\end{array}$ & $\begin{array}{l}\text { Business model } \\
\text { development. } \\
\text { Continued Agile } \\
\text { MVP } \\
\text { development. }\end{array}$ & $\begin{array}{l}\text { How difficult it } \\
\text { will be to persuade } \\
\text { international } \\
\text { distributors to trial } \\
\text { selling products. } \\
\text { If it will be cost- } \\
\text { effective for } \\
\text { carrying out R\&D } \\
\text { in New Zealand. } \\
\text { If it will be cost- } \\
\text { effective shipping } \\
\text { products from } \\
\text { New Zealand. }\end{array}$ \\
\hline $\begin{array}{l}\text { 4-Operational } \\
\text { planning and } \\
\text { development }\end{array}$ & $\begin{array}{l}\text { Carry out a } \\
\text { comprehensive } \\
\text { technical and financial } \\
\text { feasibility analysis of } \\
\text { the proposed MVP. }\end{array}$ & $\begin{array}{l}\text { A significant } \\
\text { amount of initial } \\
\text { MVP R\&D can be } \\
\text { done in-house } \\
\text { without external } \\
\text { funding. }\end{array}$ & $\begin{array}{l}\text { Network operator } \\
\text { feedback and } \\
\text { Agile development } \\
\text { of the proposed } \\
\text { MVP. }\end{array}$ & $\begin{array}{l}\text { How much } \\
\text { external funding } \\
\text { will be required if } \\
\text { external R\&D is } \\
\text { required. }\end{array}$ \\
\hline
\end{tabular}




\section{Table 2 - Project development phases.}

It is critical to note that this table was written prior to designing an appropriate MVP based on the results of data collection.

\subsection{1 - Project Scope}

This research aims to explore and develop technology that will help improve remote and rural broadband network sustainability. Furthermore, promoting methods of lowering the barrier to entry for building remote and rural Internet infrastructure throughout the MVP development process will be explored. The scope of this exploratory research is to formulate and test assumptions of the proposed MVP based on data-driven evidence, examine product and market opportunities against this evidence, and lastly, to develop a comprehensive business case for the proposed MVP. This project report may not include comprehensive technical testing of the proposed MVP, as it falls outside of scope for what is required. Full technical design and implementation details of the proposed MVP may also be omitted if necessary, due to commercial sensitivity.

\section{9 - Summary}

Building sustainable remote and rural broadband networks is a broad, complicated topic. Social, technological, and business-related issues all coexist, and there will never be one approach to solve them all. Despite little academic literature existing on building sustainable WISP networks, this chapter has identified, through literature, the necessity to develop lowcost, low-power and low barrier to entry network equipment for WISPs. Additionally, the need for wider-ranging research into network sustainability was identified. Key innovator Ubiquiti Networks was introduced, as was how its innovative business model could be adapted by Venture Networks and applied to the proposed MVP. Finally, key project objectives, assumptions, and unknowns were introduced. 


\section{Chapter 2 - Methodology}

This chapter presents the methodology employed in the research and development of the MVP. The earlier literature review identified, amongst other things, the need for robust, low barrier to entry networking equipment to be developed for WISPs. Initial findings suggest that the most significant opportunity for product development will be in targeting underserved remote and rural markets. Essentially, it is likely that assumptions will need to be tested in the context of WISPs who are looking to start, just starting out, or those who are struggling to scale their networks. Secondly, for the sake of accessibility, New Zealand WISPs were initially selected for interviews for this research project. Upon completion of the initial interviews, advocacy organisations, network vendors, consultants and other WISPs were contacted irrespective of their location.

It is also important to note that at the time of initial interviews, no MVP had yet been conceptualised. Minimising external ideas spreading into development was important to ensure that interviewer bias, and confirmation bias would be kept to a minimum throughout the data collection process. Ultimately, failing to keep the product and market validation as objective as possible could potentially result in financial loss for the research partner, Venture Networks. Therefore, keeping product and market validation as objective as possible was a vital goal of this research.

A total of 34 in-depth interviews were conducted for this research, with numerous other informal interactions enabling observations to be made ${ }^{1}$.

\footnotetext{
${ }^{1}$ Ethics approval was granted by the Victoria University of Wellington Human Ethics Committee to carry out this research - approval number 26140
} 


\section{1 - Assumptions}

The researcher has observed the behaviour of entrepreneurs attempting to launch WISP networks over several years. These networks range from well-financed start-ups to small operations run by a single person, often with a limited background in designing and implementing anything other than a simple home or office network. The most frequently recurring observation is that these entrepreneurs often ask, what could be considered simple, networking questions. This suggests that such entrepreneurs often lack the knowledge to launch and maintain high-performance, high-reliability, scalable networks. Based on the observations, several assumptions regarding product research and development have been made:

\section{Assumption 1}

Many WISP start-ups lack technical experts that have the knowledge to build high-reliability, high-performance, and scalable networks from the outset.

\section{Assumption 2}

There is an opportunity to simplify issues identified by Assumption 1 .

\section{Assumption 3}

Established providers also often suffer from limited technical knowledge, and struggle to scale their networks (Hasan et al., 2015).

\section{Assumption 4}

Low budgets and low regional income are critical issues for building and scaling highreliability, high-performance remote and rural Internet networks. 


\section{Assumption 5}

WISP network operators interviewed will be receptive to testing the proposed MVP if they see value in doing so for their business.

\section{Assumption 6}

Any MVP developed will be an original product, not an imitation.

It was also questioned if large, well-financed network operators would see the value in the lower barrier to entry networking products for their businesses. Finally, during the initial development of the business model, it was assumed that any product development would later be continued by the team at Venture Networks. Therefore, technical capability of the staff at Venture Networks was taken into consideration throughout MVP development.

\section{2 - Research participants}

To test the assumptions listed above, and to gain new insights, a wide range of telecommunications industry stakeholders needed to be interviewed. It was decided early on that primarily using qualitative research would be appropriate for this task. Rather than seeking to confirm a hypothesis about rural telecommunications network design, this research aims to explore, understand, and explain methods of building sustainable networks for the sake of evaluating the innovation, and commercialisation potential of the subsequently proposed MVP. It was also recognised that rather than being highly structured, research requirements were likely to evolve, meaning flexibility was essential. These requirements were very exploratory by nature, making qualitative research an ideal fit.

\subsection{1 - WISP network operators}

In-depth interviews were designed for WISP network operators. Open-ended questions were used to encourage interviewees to provide further depth in their responses. It was also theorised that better response rates would be attained using in-depth interviews, as opposed 
to impersonal surveys being sent through email or post. The first contact with these network operators was generally made over email or social media, and was always done in a very personal manner, in the hope of consistently eliciting a response. It was also recognised that while focus groups would have been useful for gaining insights, the sizeable geographic divide between network operators would have made facilitation of such a method impractical. The interview questions and consent forms used for WISP network operators can be found in Appendix 1 - Information Sheet for Interview Participants and Appendix 2 - Interview Questions. The length of these in-depth interviews was designed to be roughly half an hour.

\subsection{2 - Telecommunication consultants}

In-depth interviews were conducted with telecommunications consultants who regularly service WISP networks. These in-depth interviews were conducted in the same manner as those conducted on WISP network operators but were less structured. Looser structuring meant consultants were free to talk about what they wanted, and on their opinions regarding the remote and rural telecommunications industry. Interviewing consultants was crucial for MVP development, as they have first-hand experience with people interested in starting and scaling WISPs.

\subsection{3 - Equipment vendors}

Equipment vendors who focus on innovative networking products were informally contacted for comment. Little information regarding future product development was disclosed from vendors to the researcher due to commercial sensitivity. Despite having no direct access to what vendors were developing, observations about existing and known upcoming products were made.

\subsection{4 - Regulators and associations}

To understand the Government rationale towards improving telecommunications infrastructure in underserved areas, the New Zealand Minister of Broadcasting, 
Communications and Digital Media, the Hon Kris Faafoi, was contacted. Advocacy organisations such as the Wireless Internet Service Providers Association (WISPA) were also contacted. In both cases, open questions and opinions were asked, with the hope of provoking discussion, and making additional research observations.

\subsection{5 - Social media users}

During the process of identifying potential interview subjects, it was identified that social media users would be a readily available data source. Several social media groups of WISP network operators exist, with a combined membership total of over 10,000 people. The researcher is a part of these groups and has observed regular posts from new members requesting help with the design and implementation of networks that they wish to build. It was theorised that observing and analysing these posts would assist in identifying features for a potential MVP.

\subsection{6 - Further developments}

Finally, following the collection and analysis of research data, reflections were made, and additional sources of useful data identified. Through industry connections at Venture Networks, several businesspeople interested in better rural Internet infrastructure, but with limited technical knowledge, were also informally interviewed. Later, several of Venture Networks' end-users who expressed interest in helping build infrastructure for the company were contacted. Further details of these interviews are covered in the subsequent sections of this research.

\section{3 - Collection and analysis methodology}

This section describes the methodology used to collect and analyse data for new product discovery and development, or MVP development. An exploratory approach to research was taken throughout the Master of Innovation and Commercialisation programme and encouraged by each Programme Director throughout the development of this research. 
Essentially, primarily using qualitative research was deemed to be appropriate for reasons mentioned in chapter 2.2 - Research participants. Grounded Theory was selected as a methodology following a comprehensive review of different qualitative research methods. Grounded Theory is an ideal methodology for this research, as an emphasis throughout the programme was placed on findings being made objectively, through data, rather than being made subjectively through the 'ungrounded' opinions of the researcher.

\subsection{1 - Grounded Theory}

Data collection techniques such as interviews, observations, and focus groups are typically used to develop Grounded Theory (University of Leicester, n.d.). However, Grounded Theory co-creator Barney G. Glaser emphasises the fact that "all is data":

"It can use any data, but obviously the favorite data to date is qualitative. While interviews are the most popular, GT works with any data-"all is data"-not just one specific data" (Glaser \& Holton, 2004).

In the case of this research, inductive, Glaserian Grounded Theory was used to develop an MVP. It is important to acknowledge that the Glaserian approach to developing Grounded Theory was taken, as opposed to the alternative, Straussian approach. While both approaches have their own merits depending on individual research requirements, the Glaserian approach enables a dynamic range of data sources to be used, which is necessary for the completion of this research. Straussian Grounded Theory is arguably less flexible, and has been criticised as being “...programmatic and overformulaic” (Cooney, 2010).

Appropriate stakeholders were identified as participants in chapter 2.2 - Research participants, and open-ended interview questions were designed and used as a data collection technique. The format of these questions was designed to discover the processes involved with developing rural broadband networks. Questions varied depending on the stakeholder 
group (i.e., network operators or consultants). Participants were encouraged to diverge while answering questions and talk about any other areas of interest where applicable. The interview questions were very loosely designed around exploring the following processes:

- What are the processes involved with starting and scaling wireless telecommunication networks in remote and rural areas? (the core phenomenon).

- What are the processes that resulted in starting a remote or rural telecommunication network/what influences this process to occur? (the casual conditions).

- What actions/processes are carried out during the early stages of design and implementation of remote and rural telecommunication networks? (the strategies).

- What are the outcomes of these processes/strategies? (the consequences).

It was also assumed that quantitative data would later be gathered as necessary to support qualitative findings. Some quantitative results are covered in greater depth in Chapter 3 Findings and analysis.

In addition to the research participants mentioned in chapter 2.2 - Research participants, Grounded Theory was also applied to the academic sources in the introduction's literature review. It is important to note that the early literature review, while useful for finding appropriate processes to study, was not necessary for completing the Grounded Theory study. Glaser and Holton (2004) went as far as saying "The pre-study literature review of QDA is a waste of time and a derailing of relevance for the GT Study". However, sources identified in the literature review would likely have been located later regardless, to be used for analysis using Grounded Theory.

The process used in this research for developing Grounded Theory can be set out in three straightforward stages: 


\section{Stage 1 - Recruitment and data collection}

During the first stage, key industry stakeholders were identified. The list of these participants is available in chapter 2.2 - Research participants. Once participants were chosen, observations were made, interviews conducted, and other sources of data examined. This stage is also referred to as theoretical sampling.

\section{Stage 2 - Data analysis}

Open coding - the first stage of data analysis. During open coding, data sources such as transcripts and emails were read, and different categories within the data were established. Different themes and ideas were identified in the data and highlighted or circled with an appropriate category. During open coding, a constant comparative analysis was also completed. Constant comparative analysis essentially checks if data fits in a category or not, to ensure consistency. If data did not fit, a different or new category was selected. Memoing was also conducted during the open coding stage of data analysis. Memos were written regarding how categories of data might explain the processes in question, effectively forming notes about conceptual connections between the identified categories. Open coding was completed when no new categories of data could be identified from new information being analysed.

Core variable development - the second stage of data analysis. Categories were examined in order to determine how they relate to each other. During this stage of data analysis, theories started to form. Relationships formed from this process were represented as comparison diagrams, which are a visual model that describes how interactions exist. In the case of this research, NVivo was used for the graphic representation of the data. Glaser and Holton (2004) state that the core variable in Grounded Theory can be "a process, a condition, two dimensions, a consequence, a range and so forth". After a core variable was identified, it became the subject of further selective data collection and analysis. 
In this research, the core variable development process was used to discover the core processes and challenges involved with building remote and rural, typically WISP, telecommunication networks. This data assisted in building an understanding of potential customers, and their specific requirements.

Selective coding - the third stage of data analysis. During this stage of data analysis, open coding had been completed. Selective coding created explanations about how theories explained the core variable. This process was completed after the core variable was discovered. Essentially, selective coding provided a full explanation of the theory developed. From this point on, further data collection was delimited and only carried out when relevant to the development of the emerging theory. Memoing was also carried out throughout the process of selective coding, assisting in identifying the conceptual connections between categories of data.

\section{Stage 3 - Sorting and writing}

The final stage of Grounded Theory was completed once conceptual saturation of the data categories was achieved. Memos were sorted to generate a conceptual framework for the hypotheses developed through the former coding and core variable development. Memo sorting was completed through examining the appropriate categories of data and properties found within memos, and then ordering them conceptually based on these categories, and what connections they had to other memos. The process of sorting the memos is what forms the theoretical outline of the writing, to avoid any preconceived idea taking hold. This process was aided through a needs analysis map that helped visualise connections between memos. Finally, Grounded Theory regarding the potential MVP and market opportunities present was written. 


\section{4 - Design rationale}

At the beginning of this research, sending out an Internet-usage postal survey to thousands of New Zealand addresses was considered. However, for this to be effective, survey responses would need to be tightly clustered geographically. The idea of using surveys was soon disregarded, as it was determined that response rates would likely be too low to achieve meaningful results. Even if this survey had targeted a specific community or geographic region, it is likely that results would still not be meaningful on a larger scale, as too many unknowns dictate circumstances across various regions. Due to this, there is no telling if results would have reflected Internet usage in remote and rural communities on a broader scale. This approach also did not account for identifying a target market for any proposed MVP. The market pain identified would likely have been regarding slow or non-existent Internet access; a topic far too broad to develop an MVP from without further, extensive research.

Despite the quantitative surveys not being used, it was identified that there is still an opportunity for future research in determining remote and rural Internet penetration in New Zealand. Little research has been carried out on this topic to date, outside of the World Internet Project, a study by AUT that identifies Internet trends within New Zealand (Crothers, Smith, Urale, \& Bell, 2016). The AUT study was conducted using telephone and online platforms, and included 1377 participants. Two years later, another, larger AUT-run study was completed, with 2012 participants, approximately 1000 of which came from an online panel platform (Techatassanasoontorn, Andrade, Hedges, \& Karimikia, 2018).

It is the opinion of the researcher that while useful for many Internet research purposes, the AUT studies are not suitable for effectively measuring remote and rural internet penetration. Experience has shown that many people situated in remote or rural areas lack a sufficiently fast Internet connection to complete such surveys and are also often without reliable cell phone coverage. Essentially, it is likely that only urban Internet penetration was measured 
effectively in this study. Making this unclear from the outset is potentially dangerous to remote and rural communities, as government regulators may develop poorly informed views of the effectiveness of existing government-funded infrastructure projects.

WISP network operators were the ideal target group to approach, as they are familiar with remote and rural areas, and are ultimately responsible for a significant portion of Internet access outside of urban centres. Experience also shows that these service providers are responsive and happy to help where they can, making them the ideal candidates for in-depth interviews. Of course, interviewing a broad range of industry stakeholders, such as government regulators, associations and equipment vendors, as well as conducting a thorough literature review was equally important.

\section{5 - Reflection and further research}

The qualitative research tool NVivo was used to assist with data analysis. NVivo allows a researcher to organise data, categorise it, and analyse it as required. NVivo is useful for many types of qualitative research and has been proven to be sufficient for use with Grounded Theory (Hutchison, Johnston, \& Breckon, 2010). The process of data collection and analysis was repeated iteratively until theoretical saturation was achieved, which concludes a Grounded Theory study. Charmaz (2013) describes saturation as being reached when data collected stops developing new theoretical insights. NVivo's memo functionality was used extensively to keep track of theoretical development throughout the iterative research process. The iterative process followed is illustrated below: 
The process of reflecting upon data analysis and making recommendations was completed in collaboration with Venture Networks. In addition, regular supervisor meetings and frequent contact with the Programme Director meant that additional insights could be integrated into the iterative development process. As any party involved in the reflections and recommendations stages of the process had feedback, it was considered, and additional data was gathered as required, and the iterative process continued. While substantially slower than a predefined and linear approach to data collection and analysis, following an iterative process enabled a deeper understanding of crucial research problems. For Grounded Theory, following the iterative approach meant that interview questions evolved based on the answers of previous interviewees.

\subsection{1 - Methodology changes}

After the decision was made to use Grounded Theory, the research methodology had little change. Perhaps the largest evolution of the research methodology was the collection of additional observations and quantitative data from social media. At the time of planning the methodology, it was acknowledged that social media groups would be useful for making observations. What was not considered at the time was how responsive these groups would be towards polls and other informal questions. When necessary, polls were put on social media 
groups to gain additional product and market insights. The social media groups were able to offer near real-time feedback to questions and propositions, which proved useful later during technical development of the MVP.

\section{6 - Product and business model development methodology}

MVP feature and market discovery were achieved through the Grounded Theory methodology explained in Chapter 2.3 - Collection and analysis methodology. It is important to recognise that this research distinguishes between the feature and market discovery processes used to the technical and business model development methodologies leveraged for developing the MVP. That is, Grounded Theory was used to discover product and market opportunities, or identifying an MVP, and an Agile methodology was utilised for the subsequent software and hardware development processes. Evidence from the Grounded Theory study was used for business model development, but the business model itself was developed iteratively as requirements changed.

"Successful innovation requires a deep understanding of customers, including environment, daily routines, concerns, and aspirations."

(Osterwalder, 2010).

The quote above echoes what the systematic processes used by this research hope to achieve: Understanding the challenges involved with remote and rural broadband connectivity, developing product and market insights, and finally, developing an innovative MVP and business model. Ultimately, this means thoroughly understanding customers and designing products based on evidence-based research, or in other words, learning what customers actually need.

Following the completion of the iterative data collection and analysis process for discovering product and market opportunities, business model development began. As mentioned in the 
introduction, the Strategyzer Business Model Canvas was used iteratively to develop an appropriate business model for the proposed MVP. Once theoretical MVP development was complete, an Agile methodology was chosen for bringing the proposed MVP to fruition. Rather than taking a linear, Waterfall methodology to development, the Agile approach enabled feedback to be gathered from industry stakeholders throughout development, which was then integrated as required. The approach formed an iterative, somewhat continuous feedback and integration loop. An illustration of what was effectively achieved is shown below:

\section{7 - Limitations}

As with any research, there were limitations and challenges encountered. There was limited access to what future solutions other equipment vendors were developing, as expected, due to commercial sensitivity. It was also often challenging having to conduct interviews with participants situated internationally. Many equipment vendors, large service providers, and consultants live internationally, often in North America. Issues were experienced on several 
occasions due to poor video and audio quality, despite trying a variety of platforms, such as VoIP, Facebook Messenger and Skype. Technical difficulties often led to confusion and sometimes shorter interviews when the quality was unacceptable. Due to this, email and Google Docs (for real-time collaboration), were used where possible.

The Grounded Theory approach used also has several limitations. Data collection can take a significant amount of time and is a very reiterative process by nature, unless an abbreviated version of Grounded Theory is employed (Willig, 2013). Analysis and accurate coding of data can also be complicated, and there is no clearly defined process for coding to be completed (Allan, 2003). Timonen, Foley, and Conlon (2018) argue that there is often confusion from students and experienced researchers alike as to whether they are applying the Grounded Theory methodology correctly. Of course, there may also be researcher bias. Glaser and Strauss (1967) stated that the researcher should have "no preconceived ideas" throughout data collection and analysis. In the case of this research project, while attempting to act as objectively as possible, an early literature review was carried out in order to find potential channels of interest. Finally, there are unknowns about the sample size used. In-depth interviews were conducted until conceptual saturation, although it must be questioned if a relatively small sample size can accurately represent the mindset of a worldwide market.

There were also challenges faced while attempting to interview WISPs New Zealand wide, due to their wide-ranging geographical locations. The sparse geographic divide meant face-to-face interviews and inspecting networks first-hand was not usually practical. The lack of access to physical networks was unfortunate. Given greater physical access to WISP network topologies, additional research observations could have been made. Additionally, while undoubtedly useful, email itself was an uncertain way of making the first contact with potential interview subjects. Due to this, careful planning was carried out to entice responses, and included offering low, $\sim 30 \mathrm{NZD}$, value gifts such as chocolate or wine for interview participation. 
Lack of access to the programme research funding that was initially offered to students became a significant limitation. Many gifts were not sent out to interview participants due to there being no money available. Gifts for some participants were paid for by the researcher or by Venture Networks, to maintain healthy working relationships for future arrangements. It also meant gifts could no longer be offered as an enticement to participate, which likely lowered the response rate of potential subjects. Ultimately, this became embarrassing to the researcher and did not uphold a good image for any party involved, nor for the credibility of the research itself. Should funding come available, gifts to participants who did not receive them shall be sent out.

Additional access to remote and rural communities that are exploring how to improve their Internet infrastructure would have been beneficial to this project report. Understanding the mindset and purchasing decisions of groups such as these will be critical to market and product validation in the long-term, should an MVP be developed and brought to market. A hands-on approach to finding these communities would be ideal, however prohibitively expensive, especially given that much of the target market is situated internationally. A greater academic exploration into understanding how to improve connectivity outcomes in remote and rural communities would also be useful to further this understanding. As briefly mentioned in the introduction, most recent publications on improving connectivity in these areas are published by two institutions, demonstrating a clear need for additional research.

Finally, access to organisations such as the University of Oregon's Network Startup Resource Centre would have been beneficial to the development of the project report. The Network Startup Resource Centre, or NSRC, is an organisation that amongst many other things, helps in the design, implementation, and running of Internet infrastructure across the world (NSRC, 2016). The NSRC would be an ideal collaborative partner for testing an MVP that has been developed. The NSRC was not approached for interview, as extrapolating market research information goes against the organizations non-profit nature. However, following the development of an MVP, the NSRC will be approached for feedback. 


\section{Chapter 3 - Findings and analysis}

Following the formulation of the methodology, this chapter describes the findings from the interviews carried out, and any additional observations that were made. Emphasis was placed on findings from the initial New Zealand interviews, as developing an understanding of local market needs was an important research objective. As a result, each key finding from the New Zealand interviews is analysed in depth. Finally, findings from the overall Grounded Theory study are presented and then analysed. The key results from this chapter assisted in defining potential features for the proposed MVP.

There are not necessarily correct or incorrect ways to build rural telecommunications infrastructure. There is also no doubt that different countries experience their own unique challenges when improving rural connectivity. However, data gathered and analysed using Grounded Theory throughout this research showed that many WISPs, both start-up and established, struggle to design and implement effective, scalable rural telecommunication networks. Results demonstrate that there is market opportunity for fundamentally changing the way that WISP network operators design and build their networks - irrespective of their size.

\section{1 - Disclaimer}

Memos created throughout the Grounded Theory collection and analysis process were not included in this research. Interview participants were guaranteed anonymity throughout the study, and some memos contain personally identifiable information. Memos that do not directly contain personally identifiable information could still be used to identify participants through other means, therefore are also omitted. Instead, overall findings of the study, and conclusions drawn between memos, are discussed. 


\section{2 - Insights from initial interviews (New Zealand)}

To the surprise of the researcher, several representatives of Spark, Vodafone, and 2Degrees were unresponsive when approached for comment on rural Internet infrastructure in New Zealand. Collectively, these companies expressed the opinion that rural Internet connectivity is largely a non-issue and that their government-funded infrastructure provides service beyond what is currently necessary. As expected, smaller, regional providers vehemently disagreed, although never wanted to go on public record to discuss why.

Open-ended, in-depth interviews were conducted with 18 New Zealand WISPs. In total, these WISPs account for approximately half of the WISPs known to currently operate in New Zealand. For maximum impact, interview requests were sent out to every New Zealand WISP that could be identified. At the time of writing, these interview requests had a success rate of $42 \%$. Following the interviews, topics discussed were coded, and memos were created to use for the development of Grounded Theory.

Before divulging the results of the overall study, some initial findings from New Zealand WISPs are discussed in chapter 3.2.1 - Categorised findings. The top two areas of challenge for New Zealand WISPs were identified as:

\section{Spectrum availability.}

2. Power availability and cost of power systems at remote sites.

Additionally, operators were consistently concerned with how government funding (RBI1 and RBI2) was allocated, and the accountability of funding recipients following allocations.

\subsection{1 - Categorised findings}

This section briefly describes the findings from interviews conducted with New Zealand WISPs. For brevity, only the top 6 categories of significance are described; however, MVP discovery and development processes are not discussed until after the results from the overall 
Grounded Theory study are analysed. Below is an illustration showing the preliminary (New Zealand) interview results:

Figure 3 - Areas of concern (NZ WISPs).

For readability, the above graph is also shown in table form:

\begin{tabular}{|l|l|}
\hline Barriers to growth/problematic areas & Recurrence across interview participants \\
\hline Spectrum & $77.8 \%$ \\
\hline Site power/solar systems & $66.7 \%$ \\
\hline Capital expenditure & $44.4 \%$ \\
\hline Competition & $44.4 \%$ \\
\hline Grant allocation issues & $44.4 \%$ \\
\hline Government intervention & $44.4 \%$ \\
\hline Spectrum cost & $33.3 \%$ \\
\hline Poor environmental protection (gear) & $33.3 \%$ \\
\hline Uneducated staff & $33.3 \%$ \\
\hline Interference & $33.3 \%$ \\
\hline Land lease agreements & $33.3 \%$ \\
\hline Wholesale Internet & $22.2 \%$ \\
\hline Bad business management & $22.2 \%$ \\
\hline Unexpected expenses & $22.2 \%$ \\
\hline
\end{tabular}




\begin{tabular}{|l|l|}
\hline Maintenance costs & $22.2 \%$ \\
\hline TICSA compliance & $22.2 \%$ \\
\hline Service unaffordability & $22.2 \%$ \\
\hline Loans/financing & $22.2 \%$ \\
\hline Natural landscape & $22.2 \%$ \\
\hline Customer education/trust & $11.1 \%$ \\
\hline Site access & $11.1 \%$ \\
\hline
\end{tabular}

Table 3 - Areas of concern (NZ WISPs).

\subsection{2 - Spectrum availability}

Radio spectrum is controlled by Radio Spectrum Management (RSM) in New Zealand, a department of the Ministry of Business, Innovation and Employment (MBIE). Below is a table of standard frequencies and their respective licensed limits as controlled by RSM under the General User Radio License (GURL). In New Zealand, anyone can use GURLs without incurring license fees. Radio Engineers can be employed to write radio licenses for other frequencies, but not for higher EIRP usage of GURL frequencies. The transmission of electromagnetic radio waves without an appropriate license is prohibited (RSM, 2017). A summary of the spectrum available without a license to the public, as of December 2018, is shown below:

\begin{tabular}{|l|l|}
\hline 900MHz Wireless GURL & \\
\hline $915 \mathrm{MHz}$ to $920 \mathrm{MHz}$ & 1 watt $(30 \mathrm{dBm})$ total EIRP \\
\hline $920 \mathrm{MHz}$ to $928 \mathrm{MHz}$ & 4 watts $(36 \mathrm{dBm})$ total EIRP \\
\hline $\mathbf{2 . 4} \mathbf{G H z}$ Wireless GURL & 4 watts $(36 \mathrm{dBm})$ total EIRP \\
\hline 2400 to $2483.5 \mathrm{MHz}$ & \\
\hline $\mathbf{5 ~ G H z ~ W i r e l e s s ~ G U R L ~}$ & $200 \mathrm{~mW}(23 \mathrm{dBm})$ total EIRP (indoor only) \\
\hline $5180 \mathrm{MHz}$ to $5250 \mathrm{MHz}$ & $200 \mathrm{~mW}(23 \mathrm{dBm})$ total EIRP (indoor only) OR 1 \\
\hline $5250 \mathrm{MHz}$ to $5350 \mathrm{MHz}$ & watt $(30 \mathrm{dBm})$ total EIRP for outdoor use (DFS \\
& enabled) \\
\hline Outdoor Use & 1 watt (30 dBm) total EIRP (DFS enabled) \\
\hline $5470 \mathrm{MHz}$ to $5725 \mathrm{MHz}$ & \\
\hline
\end{tabular}




\begin{tabular}{|l|l|}
\hline $5725 \mathrm{MHz}$ to $5850 \mathrm{MHz}$ & 200 watts (53 dBm) total EIRP (point to point only) \\
\hline $5725 \mathrm{MHz}$ to $5875 \mathrm{MHz}$ & $\begin{array}{l}4 \text { watts }(36 \mathrm{dBm}) \text { total EIRP (point to multipoint } \\
\text { only) }\end{array}$ \\
\hline $\mathbf{2 4} \mathrm{GHz}$ Wireless GURL & 1 watt $(30 \mathrm{dBm})$ total EIRP \\
\hline $24 \mathrm{GHz}$ to $24.250 \mathrm{GHz}$ & \\
\hline $\mathbf{6 0 ~ G H z}$ Wireless GURL & 20 watts $(43 \mathrm{dBm})$ total EIRP \\
\hline $57 \mathrm{GHz}$ to $64 \mathrm{GHz}$ & $316 \mathrm{~kW}(85 \mathrm{dBm})$ total EIRP (point to multipoint \\
\hline $57 \mathrm{GHz}$ to $64 \mathrm{GHz}$ & only) \\
\hline 64 to $66 \mathrm{GHz}$ & 20 watts $(43 \mathrm{dBm})$ total EIRP (indoor only) \\
\hline
\end{tabular}

\section{Table 4 - General User Radio License (GURL).}

For outdoor use over long distances, $5 \mathrm{GHz}$ is the most effective frequency for New Zealand WISPs to use. The 200 watts maximum EIRP offers WISPs the ability to establish and operate relatively fast, long-range outdoor wireless backhauls and point to multipoint links with a wide range of channels to choose from. $5 \mathrm{GHz}$, however, is sensitive to obstacles in the lineof-sight between transmitting and receiving radios. Alternatively, $2.4 \mathrm{GHz}$ is less sensitive to obstacles in the line-of-sight over short distances. However, the maximum EIRP under the GURL for $2.4 \mathrm{GHz}$ radios is much lower, effectively limiting use to line-of-sight applications regardless. Additionally, $2.4 \mathrm{GHz}$ radios inexpensively available today are much lower performance than $5 \mathrm{GHz}$ radios, and there is limited bandwidth to use in the $2.4 \mathrm{GHz}$ frequency. $900 \mathrm{MHz}$ is another option for non-line-of-sight links; however, it also has limited bandwidth available, and low EIRP limits. Resultantly, $5 \mathrm{GHz}$ is the most robust choice for WISPs in most circumstances.

Due to crowded spectrum, some equipment vendors are now turning to $60 \mathrm{GHz}$ for short-tomedium range outdoor point to multipoint deployments. $60 \mathrm{GHz}$ offers exceptional throughput over short distances, but is very sensitive to rain fade and obstacles in the line-ofsight between transmitting and receiving radios (Singh, Prasad, \& Bonev, 2018). In commercial $60 \mathrm{GHz}$ products today, the maximum link distance recorded with commodity hardware is currently $2.5 \mathrm{Km}$ (IgniteNet, 2018). While not useful in long-distance point to 
multi-point links, $60 \mathrm{GHz}$ is a promising technology for WISPs who want to deploy gigabit or 10-gigabit Ethernet services in urban environments.

It should be added that for long-range wireless links, simply having a line-of-sight between transmitter and receiver will not ensure link functionality. There must also be Fresnel clearance between the links. As electromagnetic waves propagate through free space in a typical point to point or point to multipoint configuration, they form an ellipse, or what is referred to as the Fresnel zone. The size of this ellipse depends on the distance of the link, and the frequency that is being used. Approximately $60 \%$ of the Fresnel zone must be clear for the link to perform without significant deterioration (Henderson, 2013). An illustration of trees obstructing the Fresnel zone is shown below:

Finally, to put EIRP limits in perspective, the maximum EIRP of a $5 \mathrm{GHz}$ point to point link is 200 watts or $\sim 23 \mathrm{dBW}$. The maximum EIRP of a $5 \mathrm{GHz}$ point to multipoint deployment is 4 watts, or a meagre $\sim 6 \mathrm{dBW}$. Contrast these limits to the point-to-point EIRP of a local power company's substation to substation wireless backhaul link. In the Horowhenua, Electra Ltd.'s Kawiu Road substation to Te Paki link operates at $11.075 \mathrm{GHz}$ and has an EIRP of 1995 watts, or $33 \mathrm{dBW}$ (Hansen, n.d.). Contrast this figure to another final example - the approximate EIRP of many cellular towers. Vodafone's cellular transmitter near Kohitere Trig in the Horowhenua operates at $952 \mathrm{MHz}$ and has an EIRP of 31,622 watts, or $45 \mathrm{dBW}$ (RSM, 2016). 
With strict regulatory limits that must be adhered to and the challenges of finding appropriate locations to build radio links, there are many factors for WISPs to consider when building their networks. On top of these regulatory factors is arguably the hardest challenge, interference. As the $5 \mathrm{GHz}$ spectrum is controlled through the GURL, it is used by nearly every home and small office router. Unfortunately, not all home routers are configured correctly. In the case of misconfigurations, which experience has shown to be incredibly common, electromagnetic noise is introduced into surrounding networks, and performance can greatly suffer. Nearly every WISP interviewed agreed that if the New Zealand Government made special frequency provisions for WISP networks, innovative wireless Internet services could be far more ubiquitous across the country.

\subsection{3 - Site power}

It was identified that within New Zealand, powering off-grid network sites (also referred to as points-of-presence, or PoPs) is an expensive endeavour. Powering sites with wind power is not consistent enough for regular use in most areas, and solar systems require considerable upfront investment. Solar power systems used at off-grid sites were found to be problematic for three key reasons:

- The high cost of batteries.

- The high cost of quality battery charge controllers.

- The high cost of quality solar panels.

WISPs also identified that enclosures used for housing equipment and batteries are often retrofitted garage storage solutions from hardware stores, making them less than ideal for prolonged environmental exposure.

\subsection{4 - Capital expenditure}

Unsurprisingly, capital expenditure was a topic that was discussed with many interview participants. WISPs are often in strong competition with other ISPs, and their service prices 
often reflect this. WISP service prices have seen further reduction given the increased coverage of competitors such as Spark and Vodafone. These large competitors also have the benefit of nationwide coverage, especially in densely populated, urban areas. WISPs, on the other hand, typically serve sparsely populated, rural areas. One observation that was identified early on in Venture Networks' operations was that rural customers do not always understand why their Internet service might cost more than it would in a city. Compared to WISPs, cellular providers typically have greater financial capability to subsidise the costs of service in remote and rural communities. This is due to urban infrastructure investments that yield considerable returns given greater population density and a much larger attainable market. As a result, WISPs that fail to build robust, low-cost infrastructure may have their business models rendered unsustainable if they fail to signup customers willing to pay more for their services.

Additionally, the cost of land-lease agreements, transmission towers, power systems, radio licenses, radio equipment, staff, and other general business expenses can be prohibitive to those looking to start WISPs. For well-established networks, financing the build-out of new network segments can still be an expensive, time-consuming task. One local provider stated that they finance everything in-house, meaning asset reliability and predictability is essential to keeping their operational expenses to a minimum. The same provider also identified wireless education as being problematic - for staff and customers alike. Customers were identified as not necessarily understanding that the wireless network technology used by WISPs is robust. Educated staff were identified as being hard to find - and time expensive to train if necessary.

\subsection{5 - Competition}

The supposed anticompetitive behaviour of Vodafone disappointed most of the WISPs interviewed following the allocation of initial RBI1 funding in 2010. Frustration was identified as not existing exclusively due to another large organisation being allocated grant 
money to build infrastructure. Instead, WISPs experienced the flow-on effects of Vodafone increasing its rural presence through what most providers referred to as dangerous business practices. New Zealand WISPs identified these as being related to:

- Difficulty forming new land access/lease agreements with regional stakeholders.

- Vodafone having poor network performance and poor accountability.

- Poor use of RBI funding.

The difficulty forming new land lease agreements becomes an issue when Vodafone, or following RBI2 funding, the Rural Connectivity Group (RCG), proposes a lease agreement to a farmer/landowner, and the farmer's lawyer informs them they should expect, e.g., $\sim \$ 20,000$ a year in return (amount provided by a WISP interviewed). When a smaller regional provider approaches the same farmer, the farmer already expects relatively high remuneration. The smaller WISP might not be able to sustain higher than usual remuneration, as their small PoP may only generate $\sim \$ 350$ per month in profit (amount provided by a WISP interviewed).

The claim that Vodafone's rural network has poor performance, and Vodafone has poor accountability to said performance cannot be verified. The best chance of objective performance reporting that rural users experiencing connectivity difficulties have, on any network, is periodic connectivity tests conducted by the Commerce Commission. Unfortunately, these tests only cover 3000 broadband connections nationwide, with an unspecified number being rural users (SamKnows, 2018). With this said, the Commerce Commission has fined Vodafone New Zealand several times for misleading consumers and false advertising, the most substantial fine totalling nearly $\$ 1$ million NZD. As of 2012, Vodafone held the record for the highest ever penalties imposed by the Commerce Commission under the Fair Trading Act, totalling close to $\$ 1.5$ million NZD over numerous court rulings (Commission, 2012).

The claim that RBI funding is not being used effectively is subjective. Take site power requirements as an example. If WISPs are using unlicensed frequencies at a remote, off-grid site, the cost of deploying a power system might range somewhere between $\sim \$ 5,000$ - 
$\$ 30,000+$, depending on a variety of environmental and technical factors. If the likes of Spark or Vodafone built a site that requires off-grid power, consumption would be greater due to the higher power requirements of their licensed radio equipment, and consequently far more expensive. Purchasing licensed radio equipment further adds expense, as it is typically far costlier than unlicensed radio hardware.

The operational case that is typically made against Spark/Vodafone/2Degrees by WISPs is that they can serve tiny remote and rural communities in a financially viable manner. Contrast this to cellular providers, who inherently have higher costs, such as licensed radios and greater power consumption. Additionally, cellular providers must hire or employ radio engineers to license their radio links. Possibly the clearest way of comparing financial expenditure between cellular providers and WISPs is the innovative methods that WISPs employ to connect customers out of their service area. If a customer is on the fringe of reception due to terrain, a WISP can sometimes install a small pole in another location nearby and power it with a small solar system. Relatively cheap workarounds such as this are much more expensive to achieve when dealing with licensed hardware and higher power budgets.

\subsection{6 - Grant allocation and the Rural Broadband Initiative}

The RBI programme was announced in 2010, with Vodafone being granted $\$ 300$ million to improve rural broadband connectivity New Zealand wide; by August 2016, Vodafone had built 154 new RBI towers and upgraded an additional 355 (Corner, 2016; MBIE, 2018). Tenders for the second phase of the Rural Broadband Initiative were carried out in 20172018. Following the tender process, The Rural Connectivity Group, comprising of Spark, Vodafone, and 2Degrees was formed, and received \$250 million NZD. Several WISPs nationwide also tendered for RBI2 funding, 9 of which were successful, and received approximately $\$ 8$ million NZD between them (MBIE, 2017). In December 2018, this funding was further expanded, with an additional \$105 million NZD allocated to expanding RBI2. Of 
this expansion, an unspecified amount has been allocated to an additional 8 WISPs (CIP, 2018).

Interviews were carried out before the December 2018 announcement of RBI2 expansion funding recipients, which may explain why WISPs were concerned about the uncertainty of future funding. Despite the general uncertainty from WISPs over how RBI funding has been allocated to date, it is fair to say the New Zealand Government is beginning to recognise that WISPs are an essential part of remote and rural communities nationwide. Also, WISPs can apply for funding through the Provincial Growth Fund if they meet requirements set out by the Provincial Development Unit (MBIE, n.d.-b). The Provincial Growth Fund is of importance to WISPs, as it states projects that go “... beyond existing broadband investments to expand the reach and enhance the quality of digital connectivity available in the regions" are of particular interest (MBIE, n.d.-a)

\subsection{7 - Government intervention}

Finally, WISPs are concerned about the possibility of future changes being made by the New Zealand Government which they feel may be difficult for them to implement. When queried about specifics, WISPs usually cited TICSA (Telecommunications (Interception Capability and Security) Act 2013) and spectrum-related changes as areas of concern. TICSA compliance is likely an area of concern that is misunderstood by some WISPs. If a network has under 4,000 subscribers, it does not have to have full lawful interception capability, but instead must be "intercept ready" (MBIE, 2013). Being interception ready essentially means WISPs that have under 4,000 customers must cooperate with law enforcement agencies, and pre-deploy delivery ports at a suitable location on their network that lawful-interception equipment can be connected to (NZNOG, 2019). For WISPs, this essentially means configuring something as simple as a port-mirror - a task that should be of little technical difficulty. 
In terms of spectrum, WISPs are generally and rightfully concerned about what future legislation changes could do to their businesses. For example, if legislation were changed, and $5 \mathrm{GHz}$ backhauls could no longer have a 200 -watt EIRP, it could be detrimental to the survival of many WISPs nationwide. Changing to licensed radios would mean massive investments for WISPs, especially in terms of solar power system upgrades at remote sites, and in the acquisition of appropriate radio hardware. With this said, it would come as a surprise if anything such as this were to happen. The New Zealand Government has effectively legitimised the use of unlicensed spectrum for remote and rural Internet connectivity by funding WISPs through the RBI2 programme.

\subsection{9 - Interpretation}

While early interviews alone were not intended to discover an MVP, they did reveal that New Zealand WISPs have uncertainty about the future. Notably, they are worried about technology governance and how new or amended legislation could change how their businesses are operated. A brief interpretation of the findings specific to New Zealand WISPs compared against research assumptions developed earlier is detailed below:

\begin{tabular}{|l|l|}
\hline Assumption & Analysis (New Zealand specific) \\
\hline $\begin{array}{l}\text { 1. Many WISP start-ups lack technical experts that } \\
\text { have the knowledge to build high-reliability, high- }\end{array}$ & $\begin{array}{l}\text { Not possible to evaluate given the relatively small } \\
\text { sample size of New Zealand WISPs used for the } \\
\text { Grounded Theory study. WISPs interviewed were } \\
\text { also well established, many later received RBI2 grants. }\end{array}$ \\
$\begin{array}{l}\text { 2. There is an opportunity to simplify issues } \\
\text { identified by Assumption 1. }\end{array}$ & $\begin{array}{l}\text { Reasons why WISPs that started and later failed could } \\
\text { not be identified. }\end{array}$ \\
\hline $\begin{array}{l}\text { 3. Established providers also often suffer from limited } \\
\text { technical knowledge, and struggle to scale their }\end{array}$ & $\begin{array}{l}\text { Observations suggest some gaps in knowledge exist - } \\
\text { networks. }\end{array}$ \\
\hline
\end{tabular}




\begin{tabular}{|l|l|}
\hline 4. Low budgets and low regional income are critical & $\begin{array}{l}\text { if channel misuse is deliberate or related to } \\
\text { educational issues. }\end{array}$ \\
$\begin{array}{l}\text { issues for building and scaling high-reliability, high- } \\
\text { performance remote and rural Internet networks. }\end{array}$ & $\begin{array}{l}\text { Interviewed, making expansion slower than what it } \\
\text { would be through grants or other funding, making it } \\
\text { a challenge, but not necessarily detrimental to } \\
\text { operations. Some WISPs interviewed later received } \\
\text { funding through RBI2, further reducing this } \\
\text { challenge. }\end{array}$ \\
\hline $\begin{array}{l}\text { 5. WISP network operators interviewed will be } \\
\text { receptive to testing the proposed MVP if they see } \\
\text { value in doing so for their business. }\end{array}$ & $\begin{array}{l}\text { Interviewees were generally excited about the idea of } \\
\text { testing a theoretical MVP. Further analysis of this is } \\
\text { product, not an imitation. }\end{array}$ \\
\hline
\end{tabular}

Table 5 - Assumptions revisited.

\section{3 - Next generation WISP networks: A Grounded Theory}

Once all interviews were complete, social media posts on two key Facebook groups were analysed. The two groups analysed were Wisp Talk and Wisp Talk (Newbies and Startups). As touched on earlier, the Facebook groups in question proved to be an invaluable resource for connecting with other WISPs and making observations. There was much uncertainty around the project assumptions following the interviews with New Zealand WISPs. The combination of interviews and analysis of discussions that was made possible through these Facebook groups provided an overwhelming amount of data - far more than necessary to test project assumptions and develop an understanding of the WISP on a broad scale. Upon analysing the interview transcripts using the Grounded Theory methodology, three key categories of interest were identified:

1. Barriers to entry - network design and implementation.

2. Barriers to entry - finance.

3. Network hardware selection. 
Across all data collected, network design and implementation were the most commonly recurring challenge areas for WISPs, suggesting there is a basis to the first assumption "Many WISP start-ups lack technical experts that have the knowledge to build highreliability, high-performance, and scalable networks from the outset." This concept also supports research carried out by Hasan et al. (2013). It was further identified that struggling with ongoing network design and scalability is not something that only start-up WISPs encounter. However, it is unrealistic to expect someone, who is likely at considerable personal risk running their business, to admit they lack some essential technical skills required for running their Internet-centric business. Due to this, it is difficult to precisely measure quite how widespread network-related difficulties are. These are findings that support assumption three - "Established providers also often suffer from limited technical knowledge, and struggle to scale their networks".

Identifying finance as being a barrier to entry for building remote and rural infrastructure came as no surprise. Many WISPs interviewed were self-funded and grew organically without the use of bank-loans or any other external funding. For start-up WISPs attempting to take out bank loans, it became clear that many banks were hesitant to lend money. It is hard to formulate objective judgements about why this may be. Banks could be doubtful of the market need for WISPs, the robustness of the technology used, or even concerned that legislation changes could render traditional WISP business models to be unfeasible. Ultimately, the cost of building telecommunications infrastructure can be high, with potentially slim returns being realised over a long period.

The third category of interest, network hardware, is tightly coupled to the first category of interest - network design and implementation. A common issue identified was that WISPs are very uncertain about what network hardware to purchase. This issue identified that any MVP developed needs to have a clear advantage, and to be a first-choice selection for its purpose. The network hardware category also related to two distinct areas of hardware: 
routing and switching hardware, and wireless radios. The choice of routing and switching hardware was often a case of "what hardware has the best functionality for the lowest price", whereas the choice of wireless radios was, unsurprisingly, related to effective spectrum utilisation, speed, and platform reliability. Given the findings, it was suggested that a prospective MVP would need to incorporate:

1. The ability to simplify network design and implementation.

2. The ability to work with existing WISP radios and client equipment.

3. Low-cost, robust hardware to operate on.

4. A clear advantage over other vendor solutions (to be an easy first choice for those starting or scaling WISP networks).

As a result, Grounded Theory began to emerge:

Start-up and established WISPs regularly lack the technical know-how to design and build highperformance, scalable networks from the outset. Consequently, network resources are not utilised to their full potential, and network performance suffers. This ultimately results in networks that cannot scale due to technical and operational inadequacies.

To support the development of the emerging theory, approximately eight months of discussions from the main Wisp Talk page were archived, coded, and analysed. The Wisp Talk (Newbies and Startups) group supplemented this with approximately three months of discussions. To help identify how data collected supports the emerging theory, NVivo was used to generate a comparison diagram between the data collected from both groups: 
Figure 5 shows the coding categories of interest (commonly recurring between both datasets/social media groups) connecting Wisp Talk to Wisp Talk (Newbies and Startups).

The categories of interest are:

1. Barriers to entry - business development.

2. Network hardware.

3. Barriers to entry - network design.

Although the finance category is not commonly recurring between the two groups, network hardware and barriers to entry - network design is. The recurring categories back the earlier 


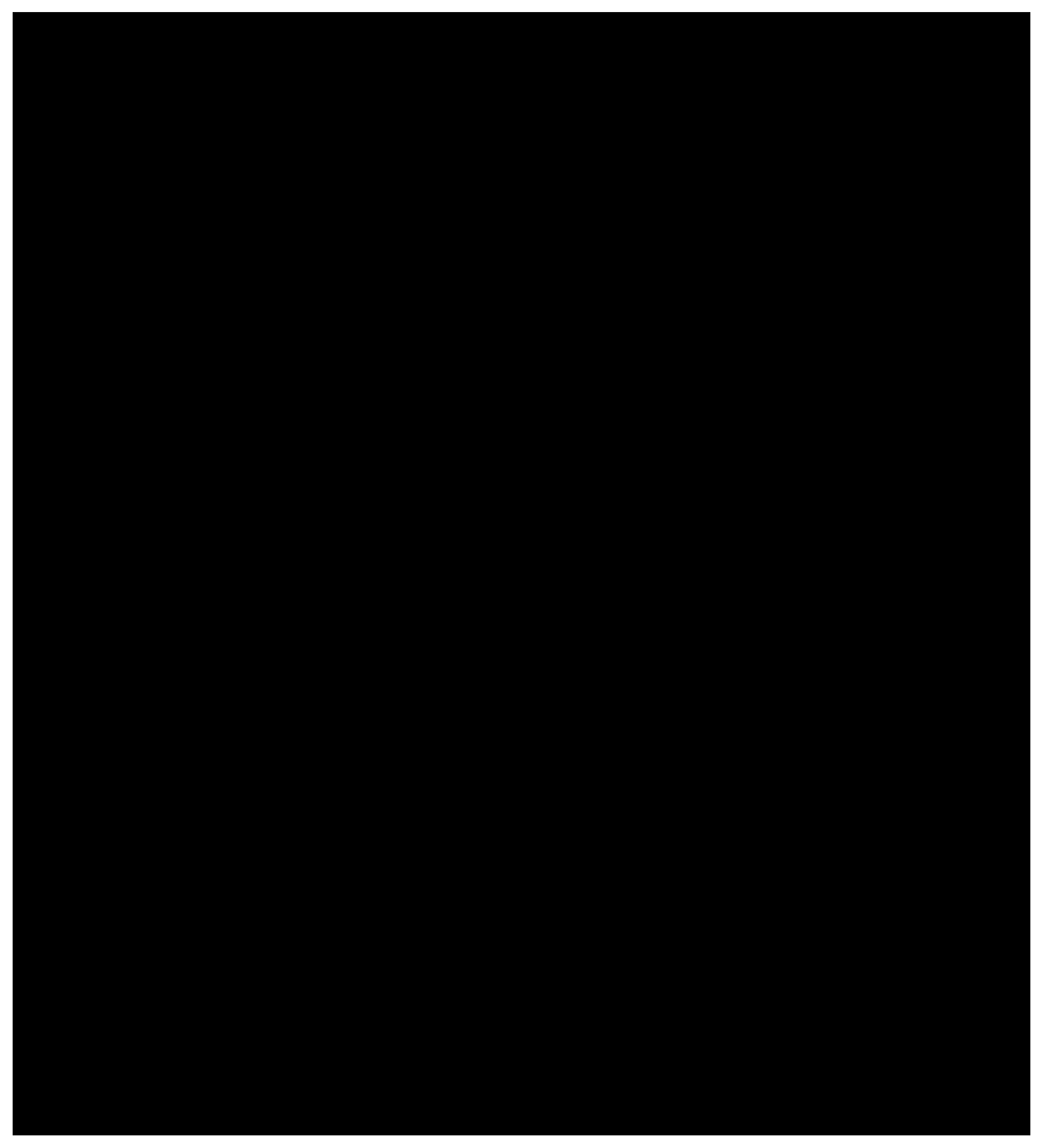


It is important to note the frequency of the words network, MPLS, and router. MPLS is an acronym for Multi-Protocol Label Switching, which is a layer-2.5 networking protocol (Steenbergen, 2010). Amongst other things, MPLS can be used to centralise the management of a network and provide point to point pseudowire services that can be used to simplify network management and routing. MPLS is a key point of discussion on both groups, and several influential consultants suggest that WISP networks, large and small, should utilise it.

Due to influential consultants, many WISPs were found to be interested in, or already implementing MPLS. For WISP operators without experience, consultants were typically hired for implementation. When WISPs hired a consultant and made a poor effort to learn how to manage and maintain an MPLS network, they often found themselves in a dilemma. It was observed that consultants nearly always emphasised that WISP operators need to "know their networks". Unfortunately for WISPs and their customers, this was found to not always be the case. While providing consultants employment, WISPs lacking the ability to manage their networks is an inherent disadvantage, especially when a network fault occurs. From these findings, it was identified that there might be an opportunity for developing an MVP that leverages the benefits of MPLS while enabling a user-friendly experience. 
The needs analysis map was formed around the three top categories of interest identified during the development of Grounded Theory. When considering interviews across all participants, and the social media content gathered, these categories were identified as:

- Network hardware (routing and switching).

- Finance.

- Network design.

The needs analysis map visualised the key areas that WISPs currently struggle in. As a result, it also shows where existing equipment vendors fall short, showing where the WISP market is 
underserved. These vendor shortcomings were used to identify what features a potential MVP requires.

\subsection{1 - Analysis}

Following the months-long development of Grounded Theory, it was shown that there is a significant market opportunity for creating a new generation of networking products for WISPs serving remote and rural areas. It was found that existing solutions to designing WISP networks at scale usually involve a plethora of complex networking protocols, most of which are not well understood by the WISP operators attempting to implement them. It was also found that while consultants play a crucial part in improving the performance and scalability of WISP networks, WISPs suffer if they become dependent on external help and cannot effectively manage their own networks.

The ability to design and implement performant WISP networks is also tightly coupled with the ability to choose appropriate network hardware, and then finance the start or growth of a network. Failing to design a robust network from early stages of operations will likely result in poor purchasing decisions being made when acquiring networking hardware. This subsequently means ineffective use of limited financial resources. Depending on the size of the network, ineffective design decisions early on and the need for later changes could be detrimental, or at a minimum unnecessarily expensive. When competing with large, wellfinanced competitors, the requirement to design an effective network on the first attempt cannot be stressed enough - for the sake of the WISP, and for the communities they serve.

With these findings in mind, the Grounded Theory developed is summarised below, and identifies that key challenge areas for WISPs are typically related to three core processes:

- The process of selecting networking hardware, whether it be related to the selection of wireless access points, CPEs, or the routing and switching hardware used in PoPs. 
- The process of financing the start-up, growth, or expansion of a WISP network.

- The process of designing and implementing a robust (high-performance, highreliability, and scalable) network.

Additionally, the discoveries mentioned throughout the findings and analysis section should be considered when thinking about future research or product development related to remote and rural telecommunications networks. These primitive, but wide-encompassing categories identify where the market pulls for WISP-related products currently are, as of early 2019. These categories will form the basis of MVP development while incorporating market needs identified throughout development of the Grounded Theory, as shown by the needs analysis map earlier.

\section{4 - Reflections}

This section briefly describes the early assumptions and reviews them given the development of Grounded Theory. Unexpected opportunities encountered, areas of further data collection and the direction of future research are also briefly covered.

\subsection{1 - Assumptions review}

To reiterate, making assumptions about research outcomes was a requirement for this project report, but was not necessary, and discouraged for the development of Grounded Theory. Assumptions were primarily based on findings from the early literature review, which was also discouraged for the development of Grounded Theory. Following the development of Grounded Theory, initial project assumptions are once again briefly reviewed:

\begin{tabular}{|c|c|}
\hline Assumption & Analysis \\
\hline $\begin{array}{l}\text { 1. Many WISP start-ups lack technical experts that } \\
\text { have the knowledge to build high-reliability, high- } \\
\text { performance, and scalable networks from the outset. }\end{array}$ & $\begin{array}{l}\text { Strong validity - confirmed. The Grounded Theory } \\
\text { developed supports early literature review findings } \\
\text { that technical competencies are a barrier to entry for } \\
\text { building WISPs. }\end{array}$ \\
\hline
\end{tabular}




\begin{tabular}{|c|c|}
\hline $\begin{array}{l}\text { 2. There is an opportunity to simplify issues } \\
\text { identified by Assumption } 1 \text {. }\end{array}$ & To be explored throughout MVP development. \\
\hline $\begin{array}{l}\text { 3. Established providers also often suffer from limited } \\
\text { technical knowledge, and struggle to scale their } \\
\text { networks. }\end{array}$ & $\begin{array}{l}\text { Plausible validity - confirmed. Even with strong } \\
\text { technical skills, some network operators lack the right } \\
\text { skills to develop robust networks. It is classified as } \\
\text { plausible because measuring the severity of this issue } \\
\text { is out of scope for this research report. }\end{array}$ \\
\hline $\begin{array}{l}\text { 4. Low budgets and low regional income are critical } \\
\text { issues for building and scaling high-reliability, high- } \\
\text { performance remote and rural Internet networks. }\end{array}$ & $\begin{array}{l}\text { Plausible validity - confirmed. Finance was identified } \\
\text { as a critical issue to building WISP networks. It is } \\
\text { classified as plausible due to difficulties measuring } \\
\text { regional rural income. }\end{array}$ \\
\hline $\begin{array}{l}\text { 5. WISP network operators interviewed will be } \\
\text { receptive to testing the proposed MVP if they see } \\
\text { value in doing so for their business. }\end{array}$ & To be explored after an MVP has been developed. \\
\hline $\begin{array}{l}\text { 6. (Product) Any MVP developed will be an original } \\
\text { product, not an imitation. }\end{array}$ & To be explored throughout MVP development. \\
\hline
\end{tabular}

Table 6 - Assumptions review.

\subsection{2- Unexpected opportunities}

Interviewing New Zealand WISPs provided not only an excellent opportunity to gather data, but also an excellent opportunity to network and build meaningful relationships. Several WISPs were very receptive to the idea of testing a future MVP prototype on their networks. While currently there is no finalised MVP to provide WISPs to test, these relationships will soon be leveraged once an MVP comes to fruition.

\subsection{3 - Further data collection}

Following interviews conducted with New Zealand WISPs, the Hon. Kris Faafoi, the Minister of Broadcasting, Communications, and Digital Media, was contacted with an overview of preliminary results. Without disclosing the full contents of the communications, it was promising to hear the New Zealand Government is actively investigating what can be done to further improve digital connectivity New Zealand wide, especially in remote and rural areas. 
This is a testament to the New Zealand Government actively pursuing different means of providing rural Internet connectivity. Shortly following contact with the Hon. Mr Faafoi, extensions to the RBI2 programme were announced, and more New Zealand WISPs received funding.

\subsection{4 - Future research}

Initially, it was hoped that contact with community broadband initiatives who are trying to or starting to build improved rural Internet infrastructure would be made. Two New Zealandbased initiatives were contacted, and unfortunately, neither replied. Future researchers approaching remote and rural connectivity issues would benefit from working with and understanding the needs of such initiatives, should they be able to identify and contact them. Successfully working with these initiatives could also help inform government decision makers in any area of the challenges involved with closing the 'digital divide'.

Future research in improving off-grid power systems, and network cognition, could greatly benefit the performance of WISP networks. In New Zealand, off-grid power systems were identified as being a key barrier to network growth, due to their prohibitive cost. Solutions to power-related issues are not immediately apparent, although being able to manage power consumption intelligently could reduce the need for unnecessarily expensive overprovisioning. Additionally, enabling radios to devise operational decisions based on the available spectrum, link-reliability, and other factors would be hugely beneficial for the performance of WISP networks.

\section{5 - MVP analysis}

If WISPs were asked what they want, they would probably say "more money", "cleaner spectrum", or "faster radios". Given the challenges identified throughout the development of Grounded Theory, it is unlikely that many WISPs would foresee a standardised, straightforward approach to designing and building robust, performant networks. A final 
survey was conducted on the Wisp Talk page following the completion of data collection and analysis, for the sake of gaining additional insights into what WISPs believe is the most challenging part of running their networks:

Results are much as expected and align closely with the Grounded Theory developed. The next step is to take findings from throughout the project report and develop a potential MVP. At this point, it should be acknowledged that for this research, developing an MVP is more than just developing an innovative product with useful features. As Eric Ries, author of The Lean Startup phrases it:

"The minimum viable product is that version of a new product which allows a team to collect the maximum amount of validated learning about customers with the least effort." (Ries, 2011).

Kromer (2014) identifies that four components of the Business Model Canvas can be used to begin the process of MVP development:

1. Customer Segments.

2. Value Propositions.

3. Channels.

4. Customer Relationships. 
An overview of the proposed MVP constructed using the four components discussed is shown in the table below:

\begin{tabular}{|c|c|}
\hline $\begin{array}{l}\text { Customer Segments - Who are our most } \\
\text { important users? }\end{array}$ & $\begin{array}{l}\text { Start-up WISPs, particularly ones that are uncertain about how } \\
\text { to design their networks. }\end{array}$ \\
\hline $\begin{array}{l}\text { Value Propositions - Which challenges } \\
\text { do we hope to solve? }\end{array}$ & $\begin{array}{l}\text { We aim to alleviate the struggle that WISPs have when it comes } \\
\text { to designing robust, performant networks. The ability to } \\
\text { operate robust WISP networks extends to what is potentially } \\
\text { significant cost-saving through lower staff training } \\
\text { requirements, less network downtime, and less dependence on } \\
\text { external assistance. }\end{array}$ \\
\hline $\begin{array}{l}\text { Channels - How are we reaching our } \\
\text { Customer Segments? }\end{array}$ & $\begin{array}{l}\text { Customer Segments targeted will primarily be reached through } \\
\text { social media platforms such as Facebook, where help groups } \\
\text { such as Wisp Talk already exist and have a well-established user } \\
\text { base. }\end{array}$ \\
\hline $\begin{array}{l}\text { Customer Relationships - What } \\
\text { established relationships do we have, what } \\
\text { relationships are needed, and what is } \\
\text { expected of us? }\end{array}$ & $\begin{array}{l}\text { We already have well-established relationships with select New } \\
\text { Zealand WISPs. However, New Zealand WISPs interviewed are } \\
\text { well established, suggesting we should approach these } \\
\text { relationships later, once a product has been developed. } \\
\text { WISP start-ups that participated in interviews will be } \\
\text { approached again through social media for the sake of MVP } \\
\text { development and testing. Meeting with (where possible) and } \\
\text { working with these WISPs will be essential for gauging how } \\
\text { effective the product is and pivoting, as necessary. }\end{array}$ \\
\hline
\end{tabular}

\section{Table 7 - MVP overview.}

Ultimately, we want to test the hypothesis that rather than hiring consultants, WISP start-ups are ready and willing to purchase next-generation hardware that simplifies building robust networks. Additionally, we know from the literature review and the development of Grounded Theory what possible features an MVP could incorporate. The next section describes a brief technical overview of how the MVP could be implemented. 


\subsection{1 - Technical implementation}

The MVP needs to incorporate features identified in the needs analysis map to alleviate challenges with the choice of network hardware, network design, and finance. A table is shown below proposing how these areas could be integrated into a platform:

\begin{tabular}{|c|c|}
\hline Challenge area and market needs & Platform integration \\
\hline $\begin{array}{l}\text { Network hardware (routing and switching): } \\
\text { - } \text { Easy customer management. } \\
\text { - } \text { Simple configuration. } \\
\text { - } \text { DC input, PoE output. } \\
\text { - } \text { Robust off-grid power management. } \\
\text { - Strong environmental protection. } \\
\text { - } \text { Low cost. } \\
\text { - Stable firmware. }\end{array}$ & 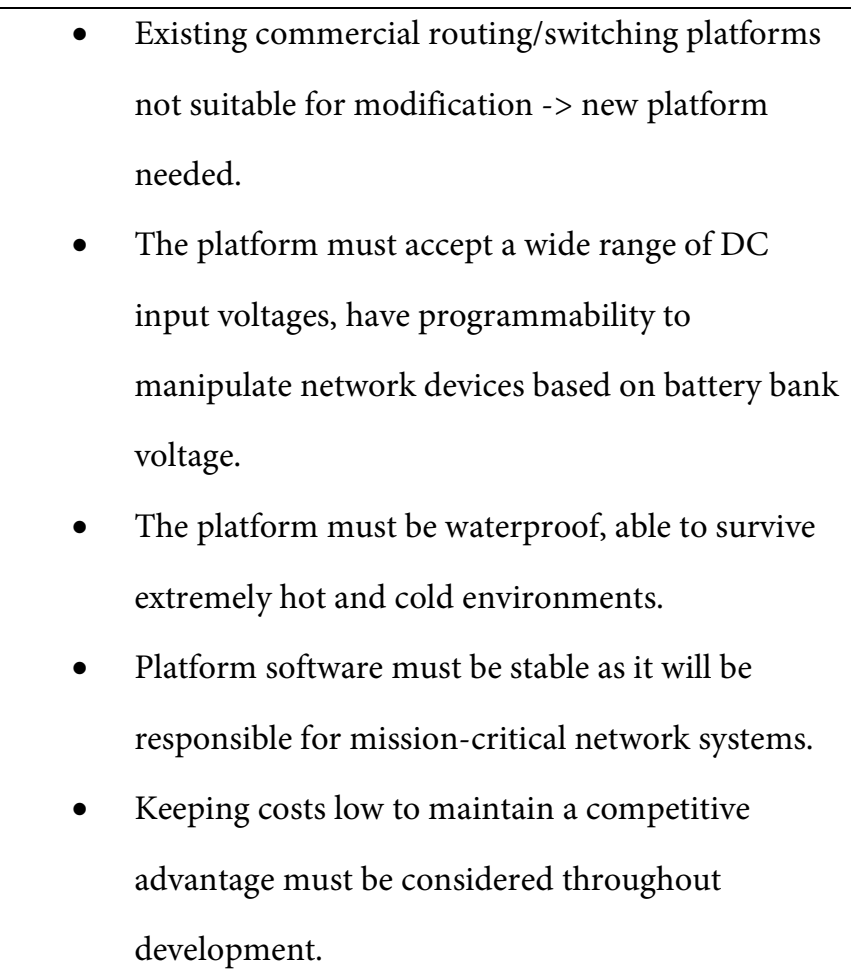 \\
\hline $\begin{array}{l}\text { Finance: } \\
\text { - Certainty of a return on investment. } \\
\text { - Predictable network operations. } \\
\text { - Investment in quality hardware. }\end{array}$ & $\begin{array}{l}\text { Finance is a critical area of challenge for WISPs } \\
\text { worldwide. The platform developed must prove it } \\
\text { can perform at a reasonable cost to be considered } \\
\text { by WISPs, especially those just starting. } \\
\text { - Platform emphasis on quality and robustness - } \\
\text { stringent testing must be carried out before mass- } \\
\text { market production. }\end{array}$ \\
\hline $\begin{array}{l}\text { Network design: } \\
\text { - Effective use of spectrum. } \\
\text { - Utilises network resources. } \\
\text { - Works with existing network } \\
\text { hardware. } \\
\text { - Predictable operations. } \\
\text { - Quality of service and performance. }\end{array}$ & $\begin{array}{l}\text { - The platform must effectively utilise all network } \\
\text { resources where possible. } \\
\text { - The platform should incorporate the "hard to } \\
\text { implement protocols" such as MPLS and OSPF, in } \\
\text { an easy-to-use manner. } \\
\text { - The platform should have a low barrier to entry, so } \\
\text { that little prior knowledge is required. }\end{array}$ \\
\hline
\end{tabular}


- Low barrier to entry (little staff knowledge and training required).

- Automation of manual processes to reduce human interaction with the network.
- The platform must work with existing wireless radios from a range of vendors, e.g., Ubiquiti, Mimosa, Mikrotik.

- The platform must have a clear advantage over existing products on the market, in terms of ease of use, and functionality.

\section{Table 8 - Market needs and platform integration.}

After discussing these platform requirements with key stakeholders, it was decided that developing a low-cost Software-Defined Networking (SDN) forwarding platform would be suitable as an initial MVP. For early MVP prototypes, a low-cost, Linux-compatible (OpenWrt or similar) network appliance would be sourced. Re-purposing a low-power network device, or even OEM router, to become an SDN forwarding platform would mean more time could be spent developing reliable software, rather than developing hardware too, which might take considerably longer. The other immediate consideration made was how to power the device itself, and the network devices that would be attached to it. It was decided that a reference PoE injector would initially be used, once again to reduce the hardware development necessary.

Software-Defined Networking was decided upon as an appropriate starting point for the MVP as it enables feature requirements, or solutions to the customer pains identified, to be realised through software development. Additionally, using an SDN protocol such as OpenFlow means no 'reinventing the wheel' needs to occur during development. OpenFlow compliant forwarding platforms such as Open vSwitch already exist and have packages for embedded Linux operating systems such as OpenWrt. The existence of such platforms enables an initial starting point that can be quickly developed. This means the complexities of development are mostly limited to the SDN controller and ensuring a smooth user experience across the platform. The core components of the proposed MVP are best visualised: 
The initial MVP developed will likely look and feel like any other network router or switch but operate in a significantly different way. The MVP also relies on an 'SDN controller' located at the central office, or main point of presence. The SDN controller in question will be packaged as free software, enabling end-users to install it on any appropriate platform of choice. As mentioned in the methodology, an Agile approach has been taken to the development of this forwarding platform. The holistic processes involved with developing the proposed MVP included iterating through several stages:

- Building value propositions and channels to market.

- Measuring how effective the channels to market are, and how customers react to the introduced MVP.

- Learning from customer reactions and relationships and tuning the product until an appropriate value proposition is developed.

Future development of the MVP platform will be continued in-house at Venture Networks, with external work being completed by contract, as necessary. The benefit of using existing, 
low-cost platforms is that much of the development time will be available for software development - making the time to market potentially much faster. This also allows for functionality to adapt as requirements and value propositions change. Rather than making significant amendments to hardware designs, the software can be modified, eliminating the experience of drawn-out waits for hardware prototypes to be manufactured. Essentially, this approach enables the platform to adapt to changing product development assumptions, and market opportunities.

\section{6 - Summary}

Throughout the findings and analysis chapter it was identified that challenges faced by those building and operating WISPs typically relate to:

- The processes of designing and implementing networks.

- The processes of selecting appropriate network hardware.

- The processes of financing network development and construction.

After presenting the results of interviews conducted with New Zealand WISPs, and the Grounded Theory study carried out, the market and product opportunities were discussed. Finally, this research report recognises there is an opportunity for developing an MVP that targets customer segments interested in, or already attempting, to start a WISP. Leveraging Software-Defined Networking, a forwarding platform specifically for WISPs was proposed. The SDN platform proposed will enable easier design and implementation of WISP networks, allowing those with little technical expertise to implement high-performance, robust, and resource efficient networks. 


\section{Chapter 4 - Discussion}

This chapter builds upon findings from Chapter 3 - Findings and analysis. A comprehensive review of how the proposed MVP will be built is discussed, as are critical aspects of the proposed MVP's feasibility. The resource requirements needed for the development and commercialisation processes of the MVP are also detailed. Additionally, the research opportunities, barriers, unknowns, and further implications are outlined. Overall, this chapter aims to inform the reader of the proposed MVPs feasibility for commercialisation, and how as a product it will compare to alternatives currently available to WISPs.

Early interviews with New Zealand WISPs identified that locally, power systems and spectrum availability are key challenges. Initially it appeared that developing better power systems or software-systems to control spectrum utilisation might be appropriate avenues for exploration. Following the broader Grounded Theory study, it became clear that designing and implementing WISP networks is a challenge for WISPs throughout the world.

Additionally, it was identified that established WISP network operators still, to some extent, suffer from poor network design and manageability. The theory that established WISP network operators struggle with network design is supported by Hasan et al. (2015) who identified the challenges that WISPs face when scaling their networks. Ben-David (2015) later identified how SDN could be leveraged to solve operational difficulties and create low barrier to entry WISP networks.

One of the key takeaways from the findings is that while there is an opportunity to simplify how WISP networks are designed, it is not possible without substantial technical development. To build scalable and performant networks, many technical, socio-economic, and educational barriers must be overcome. Before continuing, it must be clarified that not all WISPs in operation suffer from the issues described above. Some large WISP networks observed have very standardised methodologies to network design, as might be expected from any other large Internet service provider. That group of large, well-resourced WISPs is still of 
interest, but would likely be a slow or non-adopter of the proposed MVP. Of course, relatively large WISP networks are of interest too, especially those who have strong customer demand but cannot manage their network assets effectively.

Based on the findings of the Grounded Theory study, the target group of relatively large WISPs will likely be run by operators that have staff with an acceptable degree of general technical expertise. However, this group of WISPs is expected to lack network subject matter experts within their companies, potentially preventing them scaling effectively without significant external assistance. In the case of these providers, socio-economic and educational factors are once again a problem. Even a well-established WISP's growth may plateau once they cannot muster resources, whether they be financial or educational, to expand their network while maintaining acceptable quality customer connections. The case of network growth reaching a plateau would be much faster for WISP start-ups that begin with limited resources. The easiest, bridged approach to WISP network design would likely plateau after $\sim 300$ subscribers, simply due to problems with broadcast traffic becoming unmanageable (Discher, 2013).

In order to build scalable, performant networks, the MVP must address how to counteract the set of technical, socio-economic, and educational barriers. WISPs with limited resources undoubtedly need a better means of building their networks. Moreover, in the case of impoverished areas with few resources, network development should not be in the form of a technically burdening, University-built network that cannot be sustained in the long-term by the indigenous population.

The MVP must consider all these factors. Whatever is built must not experience the same pitfalls as every alternative solution on the market today. It must be user-friendly, performant, and not require months of training to operate. With this said, it is essential to acknowledge that there is no 'one size fits all' approach to building remote and rural broadband networks. Different WISPs have different requirements and understanding these 
will be a process that extends far beyond the scope of the Grounded Theory study carried out. However, what the developed Grounded Theory does show is how many WISP networks, performant or not, are designed. Understanding the network design rationale of WISP operators helps to explain how existing networking products could be changed, to improve their technical and financial accessibility.

Expanding upon the earlier description of the proposed MVP, the Business Model Canvas was used to develop an appropriate business model for the proposed platform. The Business Model Canvas was completed with Venture Networks' development capabilities in mind and tailored towards business operations being run from the Horowhenua. The result was developed following the completion of Chapter 3 - Findings and analysis, and is shown below: 


\section{1 - Resource requirements}

As described in Chapter 2.1 - Assumptions, the continued development of the proposed MVP will be carried out by the team at Venture Networks. Two full-time engineering staff have already been tasked to technical development of the MVP following the completion of this research, with more planned as necessary once technical feasibility is established. The researcher also believes that the development of the proposed MVP meets the New Zealand Government's funding criteria under the Provincial Growth Fund. Venture Networks intends to apply for a grant to fund a feasibility study of the proposed MVP. If the proposal is successful, a large-scale physical testbed of the proposed MVP will be deployed across the Horowhenua region, providing infrastructure services beyond existing RBI1 and RBI2 investments.

\section{2 - Feasibility}

The two full-time engineering resources devoted to the technical development of the MVP will ideally yield a functional prototype within the next three months. The time to market for the proposed MVP is, at this stage, difficult to accurately estimate. The technical development of the proposed MVP leverages an Agile approach and incorporates aspects of the Lean Startup methodology. The use of the Agile development methodology also helps reduce the necessary technical development time, ideally meaning the proposed MVP will be brought to market faster. The resources that have been allocated by Venture Networks to the continued, future development of the MVP outside of this research are sufficient to complete necessary commercialisation processes. However, other feasibility challenges will be detailed in the following section.

\subsection{1 - Supply feasibility}

A supply agreement has been formed with a well-established Chinese contract manufacturer. The manufacturer will provide the initial hardware platform (the proposed MVP) and has the 
manufacturing capability to meet increasing demands as required. Supply of the poweringequipment for the proposed MVP hardware platform is still being investigated. Several avenues for manufacture exist. Reference hardware can be purchased from Texas Instruments directly or from other electronics suppliers such as Mouser or Element14. The poweringequipment also uses parts that can easily be hand soldered so that initial prototypes may be manufactured in Levin, at Venture Networks' workshop. Due to the scalability of manufacturing and quality control, future iterations will have manufacturing outsourced, unless funding can be secured through the Provincial Growth Fund to perform manufacturing locally.

\subsection{2 - Resource feasibility}

Venture Networks has a strategic advantage for the development of the proposed MVP, as it already has some key resources that can be leveraged. Venture Networks owns a 400 square metre facility in Levin, which includes a climate and noise-controlled server-room with substantial backup power capacity. There is also extensive free space to expand current operations, should manufacturing be performed locally in the future. Finally, as briefly mentioned earlier, Venture Networks also already employs engineering staff who have been tasked to the future development of the proposed MVP. Compared to a start-up that must build a team from the bottom up, and lease office space or a workshop, Venture Networks is in an excellent position to complete development with minimal additional overheads necessary.

\subsection{3 - Concept feasibility}

The proposed MVP utilises SDN and aims to provide a seamless approach to designing highperformance, robust WISP networks. Additional to the previously mentioned academic papers, the concept of using SDN to build robust wireless networks is supported by Detti, Pisa, Salsano, and Blefari-Melazzi (2013). Furthermore, the Grounded Theory study conducted supports the initial assumption that WISPs struggle to design and implement 
robust networks, suggesting there is a market opportunity for the proposed MVP. Despite the existence of favourable evidence, the feasibility of the proposed MVP will be confirmed or otherwise following the completion of development and trials on real-world WISP networks.

\subsection{4 - Distribution feasibility}

Distribution of the proposed MVP is an area that is still being explored. Direct sales will be offered to New Zealand WISPs, however, the feasibility of shipping products from New Zealand to foreign destinations is currently being assessed. If products cannot feasibly be distributed from New Zealand, manufacturing will likely need to be outsourced entirely, with only research and development of existing and future products being completed locally. Following the successful completion of MVP development, partnerships with international suppliers will need to be established. Until these partnerships can be developed, Amazon is a straightforward platform that can be leveraged to ship to North American customers. Amazon offers a Fulfilment by Amazon (FBA) service. Essentially, inventory can be shipped to an Amazon warehouse and subsequently distributed through their global platform. Shipping to other markets may be more cost-effective through working with the Chinese manufacturer to offer drop-shipping services, although these arrangements are still being developed.

\subsection{5 - Financing feasibility}

The development of the MVP has been funded through Venture Networks' primary cashflow, which is Internet service subscriptions. Ample funding has been budgeted to the initial development of the proposed MVP to see it through to functional completion. This includes funding for staff costs, the purchase of necessary hardware to build the platform, manufacturing costs incurred from the Chinese manufacturer, and any unexpected expenses, as necessary. Additional feasibility study funding will be sought through an application to the Provincial Growth Fund, which would enable developing a testbed for the platform across the rural Horowhenua. In turn, the testbed would provide greater access to rural broadband 
connectivity for residents in the Horowhenua, and ideally, additional jobs to support the development of the platform.

\subsection{6 - Other considerations}

Working with WISPs and consultants who deal with WISPs will be an essential step in promoting the proposed MVP. To maintain financial feasibility, it would be beneficial to promote the proposed MVP through word of mouth where possible. Initially, working with WISPs and consultants, at no cost to them, will be an essential step in establishing a name for the product. Teaming with consultants to create brand ambassadors or product champions will also be explored, as understanding the evolutionary nature of how WISP networks are run is of crucial importance to the long-term success of the proposed MVP. Ultimately, understanding customers and their needs are what must be continuously examined, and this will be greatly assisted through working with those who work with WISPs every day consultants.

\section{3 - Opportunities}

Upon successful completion, the proposed MVP will be the first of its kind to market. The evidence-based design of the MVP also gives it a definite competitive advantage, as it builds upon areas where WISP operators currently struggle. We know that the design of WISP networks varies depending on the technical expertise available. We also know that network design ranges from basic bridged networks through to networks running dynamic routing protocols, which often utilise MPLS and are of greater complexity. Finally, we know the proposed MVP takes the best features from complex, performant networks and makes them as simple as running a simple bridged network.

Venture Networks is also in an ideal position to test the proposed MVP, as it already operates a rural broadband network. The researcher has also formed good relationships with several WISP operators through the development of this research, all of whom have been receptive to 
the idea of testing a potential product. Working with and testing the MVP with other providers will form an essential part of the Agile development process, as their feedback will be fed directly back into MVP development. The possibility of accessing Provincial Growth Fund financing for this project is also an opportunity. If satisfactory funding is secured, the manufacturing and distribution of the MVP could occur locally, creating more jobs within the Horowhenua.

Another significant opportunity discovered is the ability to improve the power systems used at remote and rural WISP PoPs. The proposed MVP will initially use a Texas Instruments designed reference power supply, to reduce the amount of initial development work required. Despite being a reference design, the Texas Instruments power supply is substantially cheaper than other options targeted towards WISPs and supports a wide range of DC input voltages. Support for a wider input voltage means that, within reason, WISPs do not need to purchase different equipment if the design of their solar, wind, and battery systems changes. It also means WISPs building an entirely new network can maintain a standardised design, or alternatively, be flexible.

\section{4 - Barriers}

While the SDN approach proposed for the MVP is promising, to implement the given requirements in Chapter 3.5.1 - Technical implementation, a significant amount of technical research and development work will need to be carried out for the MVP to be successful. It is crucial that the MVP is easy to use, as much of the value to prospective start-up WISPs will be achieved through easy usability. For the usability criteria to be met, the proposed MVP should behave with a plug and play nature; building this plug and play nature into the proposed MVP while maintaining performance and reliability is a critical challenge.

Once more, it has been established that WISP networks are either bridged or routed. Bridged networks are more straightforward for WISPs to build, as they typically plug and play, with 
minimal configuration necessary. However, broadcast traffic is especially problematic in large, bridged WISP networks, as it wastes precious network resources. The broadcast traffic from one customer on a WISP network (pictured at the bottom right) being sent across the WISP network is illustrated below:

It is easy to imagine the impact this has when there are several hundred customers, each of which has (at a minimum) one device that is sending broadcast traffic across the entire network. An even worse scenario is when an inexperienced WISP configures their entire, potentially sizeable network as a bridge, and then configures their client-side equipment in a bridge mode. If the WISP is running a central DHCP server and no proxy ARP, which is likely in this configuration, each client-side device will send broadcast traffic across the entire network. The solution to this is to route individual tower sites, as illustrated: 
The broadcast traffic from the client-side equipment is then limited to being sent within the routers broadcast domain, that is, devices connected to that router. The router will eliminate broadcast traffic from being sent across the entire network. However, routing the network adds management complexities, and requires the operator to learn about routing and subnetting. The process of learning and practising routing and subnetting takes time and potentially money, making it challenging in areas where resources are scarce. The benefits of layer-3 routing as opposed to large layer-2 bridges are clear, and not just because of how broadcast traffic is handled. Layer-3 routed networks are very scalable and perform well when set up correctly. After all, the global Internet network is built utilising them.

However, the inception of SDN, and particularly Open vSwitch and OpenFlow, means that many of the benefits of layer- 3 routed networks can now be brought to layer- 2 networks while maintaining straightforward network usability. With this said, large-scale, robust layer2 networks are not widespread, and are an active research area. An example of a successfully deployed, scalable layer-2 network, is the German community wireless network Freifunk. Alongside a community of global developers, Freifunk members develop the B.A.T.M.A.N 
protocol, which is designed for use on mesh networks (such as Freifunk itself). The B.A.T.M.A.N protocol has a layer-2 implementation known as batman-adv, which has its own loop avoidance mechanism and does not require STP, allowing it to utilise available network resources effectively (Open-Mesh, 2010).

The IEEE also developed IEEE 802.1aq, known as Shortest Path Bridging or SPB. The SPB protocol eliminates the necessity of running STP, and enables multi-path transport, effectively enabling much larger, high-performance layer-2 networks (Luo \& Suh, 2011). Finally, the European Union is sponsoring a project known as Behavioural Based forwarding or BEBA. Switches designed to run the BEBA platform can perform stateful traffic processing, eliminating the dependence on external SDN controllers (BEBA, 2019). The BEBA concept is relevant to this research project, as controller unreliability is a factor that must be well accounted for in the case of WISP networks, where wireless links are not necessarily predictable.

None of the previously mentioned layer-2 solutions represents what is envisioned for the proposed MVP, although the use of B.A.T.M.A.N on network nodes for resiliency, combined with SDN-based traffic engineering is an area that will later be explored, however is outside the scope of this research. While good starting points for research and development of the proposed MVP exist, a substantial amount of technical work will be required from the ground-up. There are numerous paths for development that exist. Extending a protocol such as B.A.T.M.A.N to support traffic-engineering is a possibility, as is extending work completed by Detti et al. (2013) and others. No matter the choice, it will be important not to diverge from the requirements set out in Chapter 3.5 - MVP analysis, to further reduce development time. 


\section{5 - Unknowns}

As mentioned earlier, the exact, final technical implementation of the MVP is still in development. The market opportunity for the proposed MVP has been identified through understanding that there is a demand for straightforward to build WISP networks. The process of building the proposed MVP to solve this requires extensive technical research, which is outside the scope of this project report itself. Due to this limitation, early prototypes of the MVP may not accurately reflect how the final product will function.

Confirmation or dismissal of the assumptions has not significantly changed since Chapter 3.4.1 - Assumptions review. Three assumptions are still being explored, and are summarised below:

- There is an opportunity to simplify WISP network design.

- Other WISPs will be receptive to testing the proposed MVP.

- The MVP developed will be an original product, not an imitation.

The first assumption may seem misplaced, as the Grounded Theory study shows there is an opportunity for improving how WISP networks of varying size are implemented. However, future research on a much broader scale is still necessary, ideally from researchers across the world. The greatest challenge may not be the development of the proposed MVP, but championing its use, and demonstrating that it is a viable platform. Therefore, the opportunity assumption will be re-evaluated following the development and trials of the proposed MVP within real WISP networks.

The second assumption is not definite either. Several good relationships with WISPs have been developed, but this assumption will be satisfied once the proposed MVP has been developed and is being tested. Finally, the original product assumption will not be satisfied until MVP development has been fully completed. Numerous technical avenues exist, and 
while original development work will be carried out, it could involve modifying existing networking solutions, rather than building new ones from the ground up.

The proposed MVP is being developed based on evidence collected throughout the research process. It must be emphasised that the development will pivot, as necessary. Pivots could occur due to a variety of currently unknown factors, with several potential pivots summarised in the table below:

\begin{tabular}{|c|c|}
\hline Type of pivot & Example \\
\hline Zoom-in & Development emphasis is put on a feature of the product. \\
\hline Zoom-out & $\begin{array}{l}\text { The reverse of the zoom-in pivot. Emphasis is put on developing more product } \\
\text { features. }\end{array}$ \\
\hline Customer segment & $\begin{array}{l}\text { The wrong customer segment might be targeted; another segment is identified } \\
\text { and re-evaluated. }\end{array}$ \\
\hline Customer need & $\begin{array}{l}\text { Customer needs may have been poorly evaluated. The MVP will need to be } \\
\text { changed or even redeveloped completely. }\end{array}$ \\
\hline Platform & $\begin{array}{l}\text { The MVP may use an inappropriate platform. Platform pivots could include } \\
\text { changing from hardware to software and vice versa. }\end{array}$ \\
\hline Value capture & The methods of earning revenue are pivoted. \\
\hline Engine of growth & $\begin{array}{l}\text { The means of bringing the product to market are pivoted. The engine of growth } \\
\text { typically refers to the mouth-to-mouth approach, paid approach (e.g., through } \\
\text { Google or Facebook ads), and finally, through sticky growth (e.g., loyal } \\
\text { customers with low churn rate). }\end{array}$ \\
\hline Channel & $\begin{array}{l}\text { The means of distributing the product. A distribution change could include } \\
\text { changing from a direct sale to distributor approach. }\end{array}$ \\
\hline Technology & $\begin{array}{l}\text { New technologies can change how the product can be developed, potentially } \\
\text { with lower cost and better efficiency. }\end{array}$ \\
\hline
\end{tabular}

Table 9 - Development pivots.

\section{6 - Prior research}

Previous research from Ben-David (2015), and Detti et al. (2013) identified the potential applications for SDN within WISP networks. Ben-David (2015) theorised how SDN could be 
used to simplify network development and identified challenges involved with building an SDN-based solution. Detti et al. (2013) implemented a framework known as wireless mesh SDN, abbreviated as $w m S D N$, which aimed to create robust wireless mesh networks. This implementation took a hybrid approach and used OpenFlow capable switches combined with the Optimized Link State Routing Protocol (OLSR); the hybrid approach taken enabled network resiliency in the case of controller unavailability, which is an important concern for SDN use within networks with scarce and unpredictable resources. Unfortunately, the wmSDN framework does not appear to have seen use outside of its original development.

\section{7 - Similar products}

No SDN-enabled products specific to WISP networks currently exist. However, enterprise SDN-enabled solutions are being championed by companies such as Ubiquiti Networks, through their UniFi product range. The UniFi range of products started as high-performance WiFi access points and evolved into a variety of products including switches, security gateways, cameras, VoIP phones, and even computer-controlled LED lighting. Commercialising a variety of easy to deploy, manage, and maintain products has seen Ubiquiti thrive, with the UniFi series realising 210\% growth and greater than 10 million devices being shipped over the last three years. Cambium Networks, a rival of Ubiquiti, has now launched their own series of 'cloud-managed' switches, offering similar functionality to the Ubiquiti UniFi range, except without the SDN label being used for marketing.

\section{8 - Product comparisons}

The Ubiquiti and Cambium SDN solutions enable the design and deployment of home, office, and enterprise networks to be substantially easier than what was realised before their existence. Rapid sales growth of Ubiquiti's UniFi series of products is a testament to the potential for SDN within remote and rural network infrastructure. To date, Ubiquiti has not released anything similar that is applicable for use in WISP networks. However, Ubiquiti has 
released several other software platforms, including UNMS, UCRM, and airControl. The table below summarises the use case for each platform:

\begin{tabular}{|l|l|}
\hline Platform & Use case \\
\hline UNMS (Ubiquiti Network Management System) & Centralised network monitoring of Ubiquiti Edge \\
(router and switching) products. Will later include \\
QoS management.
\end{tabular}

\section{Table 10 - Ubiquiti software platforms.}

If Ubiquiti were to implement an SDN solution for WISP networks, they could conveniently extend the user interface on any of their platforms to do so. With the addition of QoS management to the UNMS platform coming soon, Ubiquiti could well recognise that SDNfeatures are beneficial for their WISP-specific series of service provider products too.

With Ubiquiti and Cambium SDN solutions in mind, it is important to note how the proposed MVP differs. It was recognised in Chapter 3.5.1 - Technical implementation that thorough network resource utilisation is crucial. In the case of UniFi switches, STP, or RSTP must be used to avoid layer-2 loops (Ubiquiti Networks, n.d.). The proposed MVP will maintain the plug and play nature of layer-2 Ethernet services, such as those offered by Ubiquiti's UniFi range, while better-utilising network resources to give WISPs a competitive advantage. It must be stressed that effective utilisation of network resources is a significant concern in most WISP networks, especially so when clean spectrum is scarce, and radio licensing fees are prohibitively expensive.

\section{9- Further implications}

Upon successful development of the proposed MPV there is potential opportunity in adjacent markets. Service provider networks other than WISPs are of interest, as are commercial entities that require traffic management solutions. The proposed MVP will enable smarter 
traffic management through high-level rule installation, and will be configured through a user-friendly interface. For example, a network operator could instruct the network to prioritise VoIP traffic over HTTP traffic, ensuring customers or business staff can make VoIP calls consistently and without interruption.

It should be noted that nearly all router operating systems, including those found on budget home routers, can manage connection quality of service, or QoS, to varying extents. The proposed MVP extends QoS further by enabling network operators to prioritise traffic uniformly across their entire network, while simultaneously enabling the use of what might otherwise be redundant, or backup, paths. The process of path selection and traffic management will be largely automated, maintaining network ease of use.

In contrast to utilising redundant paths, Spanning Tree Protocol (STP) and Rapid Spanning Tree Protocol (RSTP) are typically used in Ethernet networks to prevent layer-2 loops. In a loopy topology, STP and RSTP will logically disable ports on switches that are responsible for causing the layer-2 loops. For WISPs, commercial entities, and anyone else that runs STP, the ability to better utilise network resources may be an immediate benefit - for the sake of better reliability and faster network convergence in the case of link failure. 


\section{Chapter 5 - Business case}

This chapter describes the business case for the proposed MVP. It has been written as a business expansion plan for Venture Networks, with the intention of being submitted to the Provincial Growth Fund to potentially secure funding for a feasibility study to be completed. As this chapter is intended for the Provincial Growth Fund, and potentially other sources of funding, some of the content from other chapters of this research has been consolidated and reused. Finally, as the business expansion plan is intended for submission to the Provincial Growth Fund, work carried out by the researcher may be referred to as being completed by Venture Networks. While the work is original to the researcher, and not to other members of the company, referring to Venture Networks is a formality, as the Provincial Growth Fund must consider what Venture Networks as a company strives to achieve. 


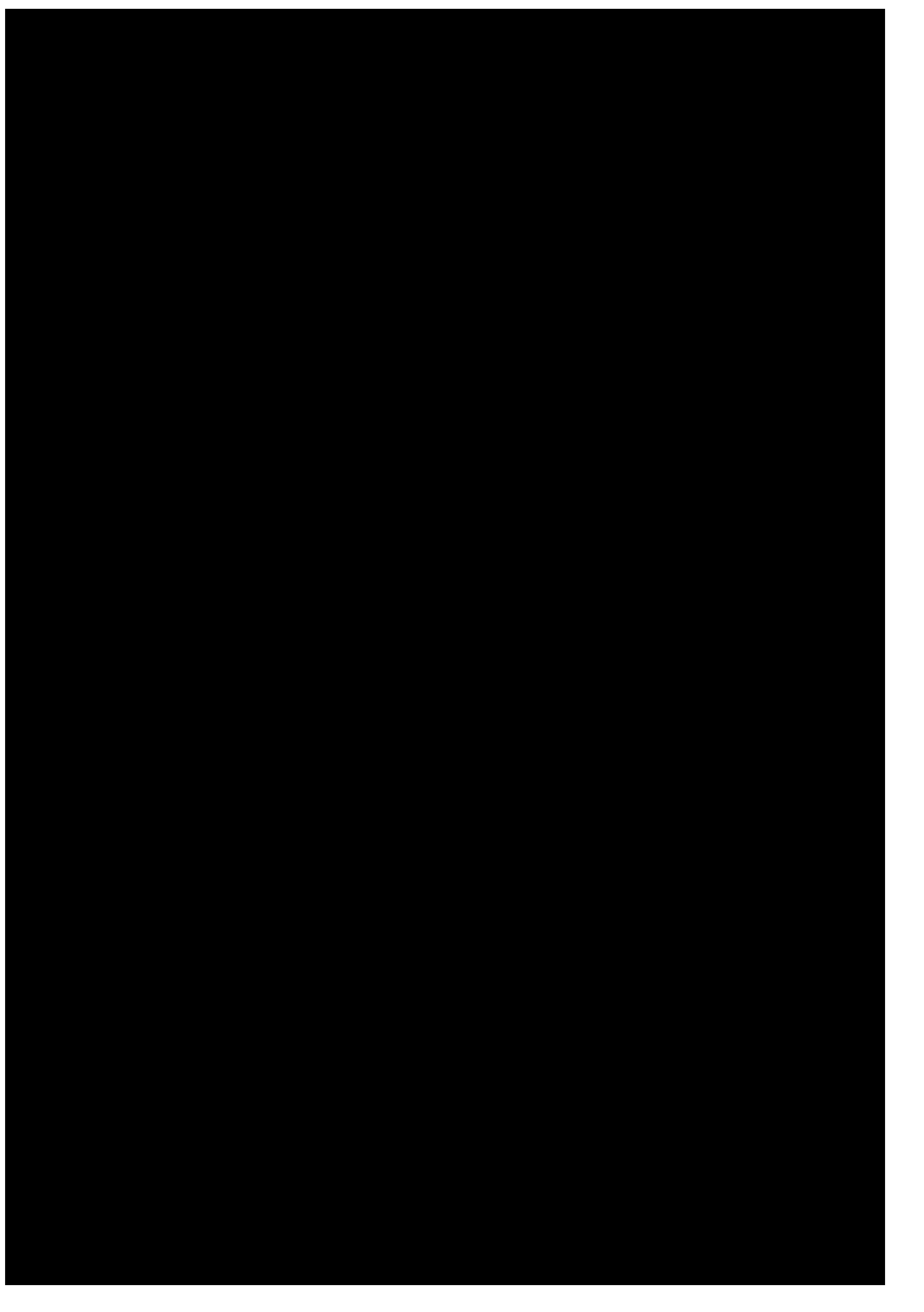


No method is more effective than a good example

Ingvar Kamprad, IKEA Founder 


\section{1 - Executive summary}

Poor access to the Internet in remote and rural areas is an afterthought for most until they experience it first-hand. Here at Venture Networks, we believe that people's lives can be digitally enriched through access to the Internet. Should it be through improved literacy and numeracy, social interactions, or leisure, we think those living in remote and rural communities deserve an equal digital opportunity to their peers in urban centres. For Internet access to improve in such communities, a sustained effort is needed from researchers, politicians, community leaders, and those living in remote and rural areas themselves. Additionally, evidence has shown us that those living in remote and rural communities need a new generation of user-friendly networking hardware.

To spur the community-driven development in these areas, Venture Networks identified an opportunity for lower-barrier to entry networking hardware to be developed. The team at Venture Networks has been developing a product that enables those living in remote and rural communities without extensive technical training to take community ownership, and build robust, performant Internet access networks. This business expansion plan outlines some key findings from the research carried out and proposes Venture Networks to seek funding from the Provincial Growth Fund to expand their physical network infrastructure. An increased network presence will enable Venture Networks to test the technical feasibility of the product developed.

Here is to better remote and rural Internet connectivity.

\section{Duncan Cameron}

Director, Venture Networks Limited 


\section{3 - Background/Overview}

Venture Networks Limited was formed in December 2013 after years of frustration with slow Internet connectivity in the rural Horowhenua district. When Venture Networks was founded, few other connectivity options existed in the rural Horowhenua. Mobile data was exorbitantly expensive, rural ADSL was too slow to buffer even low-definition videos, and high-speed copper VDSL services were out of range. After fibre optic broadband became available in Levin through the New Zealand Government's Ultra-Fast Broadband (UFB) programme, Venture Networks ordered a business-grade connection and approached a local farmer for land access to build a small wireless base station. Years on, Venture Networks has a growing customer base and constant demand from prospective clients to be connected. 
Unfortunately, slow connectivity still plagues much of the region. The RBI1 and RBI2 schemes have enabled connectivity where limited other options exist, although services provisioned through mobile $3 \mathrm{G}$ and $4 \mathrm{G}$ networks sometimes lack the flexibility to serve those most in need. Venture Networks operates what is referred to as a Wireless Internet Service Provider, or WISP network. Like many other WISPs around New Zealand and the world, Venture Networks operates primarily using $5 \mathrm{GHz}$ radios, incurring no license fees. Compared to licensed radios used by mobile operators, $5 \mathrm{GHz}$ radio equipment is affordable, uses minimal power, and can be deployed creatively to connect those without Internet access.

Venture Networks has ambitions to expand beyond providing rural broadband access. It has been observed by staff at Venture Networks that similar WISP initiatives in communities across the world often struggle with a lack of technical expertise. With scarce financial resources, poorly informed technical decision making can be detrimental to the operations of a WISP. Without well thought out network planning, WISPs frequently experience poor network performance, and consequently, poor customer satisfaction. Resultantly, rates of customer attrition increase, and WISPs may never experience the critical mass needed to break even on their network investments. For WISPs that fail to balance their books, bankruptcy or selling assets to competition at well below market rates is common practice.

Based on these observations, it is easy to speculate that there is something inherently wrong with how WISP networks are operated. In some cases, such speculation may be correct. There is a relatively narrow set of circumstances where operating a WISP is feasible. Existing competition, population density, available spectrum, legal regulations, backhaul/wholesale Internet, and terrain are all factors that must be considered by a prospective WISP operator. Additionally, it does not make sense to operate a WISP without first developing wellgrounded technical competencies. However, in developing or impoverished regions, technical expertise is not always available. Even in developed countries such as New Zealand, there is no guarantee a prospective community will be served by a WISP operator that genuinely understands their market, or the technical requirements necessary to serve it. 
To encourage the ubiquitous development of remote and rural broadband infrastructure,

Venture Networks wished to explore what commercial opportunities for helping to start and expand WISP networks may exist. A (Glaserian) Grounded Theory methodology was employed to study and understand the challenges faced by WISPs in New Zealand and abroad. The study identified the challenges, or market pains, that WISP operators regularly struggle with, and proposed a Minimum Viable Product (MVP) designed around the findings. The subsequent sections of this business expansion plan cover the innovative aspects of the MVP and the commercialisation potential that it may have. 


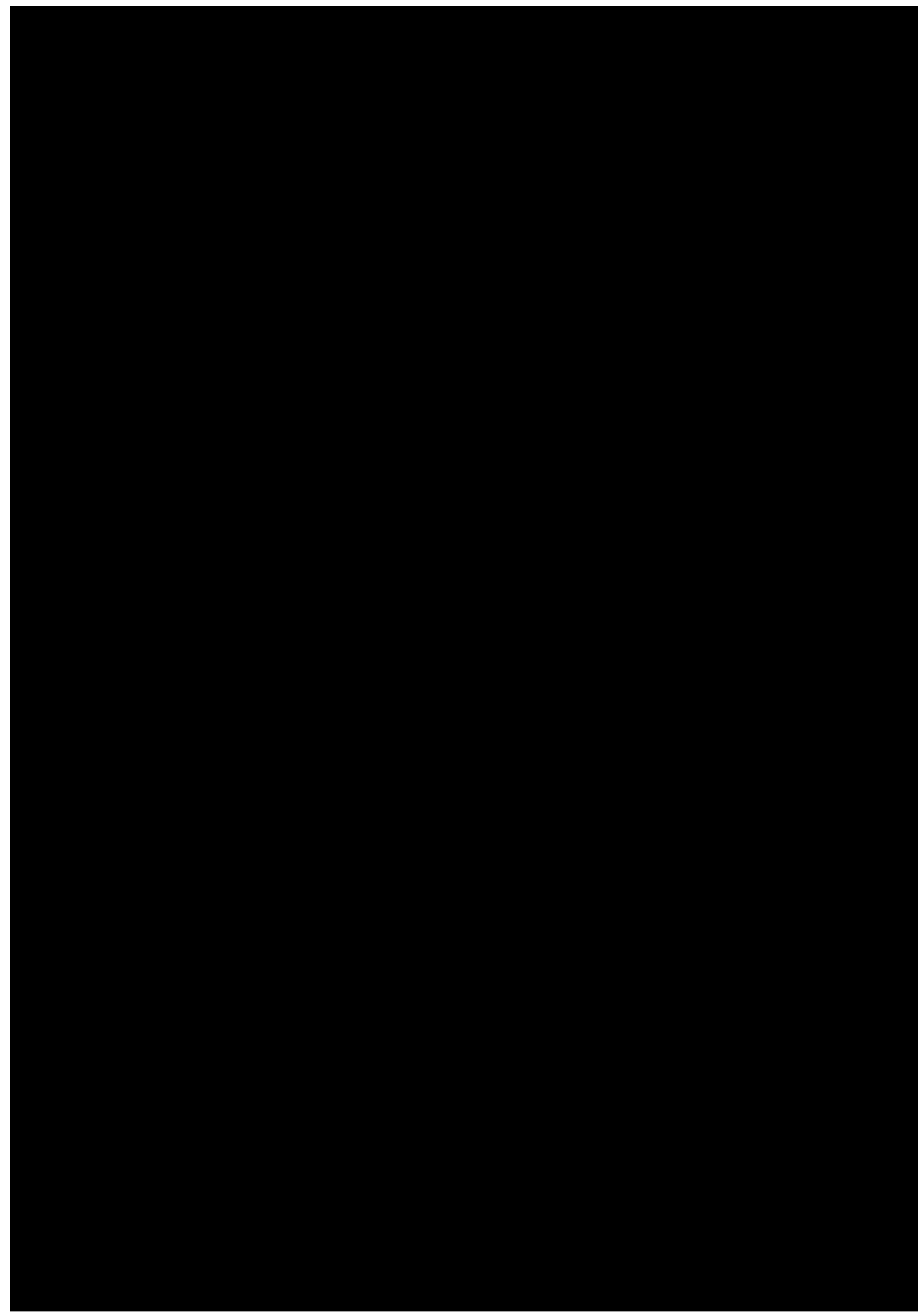




\section{4 - Product development and validation}

Development of the MVP followed the conclusion of the Grounded Theory study. Results of the study were discussed with several key stakeholders, including Venture Networks, an Advisory Board, the researcher's supervisor and other remote and rural broadband connectivity experts from New Zealand and the USA. Based on the feedback from these stakeholders, potential MVPs were drafted iteratively until an appropriate solution was identified, and Venture Networks decided to pursue development. At present, the MVP is still being developed and will require feasibility testing to further assess its commercialisation potential.

Before beginning the Grounded Theory study into WISP network operators, a brief literature review was carried out. Blantz and Summer (2011), Ben-David (2015), and Nungu et al. (2012) recognised that low-cost, low-power, and user-friendly networking equipment is necessary for the development of broadband access networks in remote and rural communities. Despite the age of the research, these challenges are still topical today. Hasan et al. (2015) expanded upon earlier research and proposed using Software-Defined Networking (SDN) to build higher-performance, robust WISP networks that are dissimilar to those found in deployments today. Years later, research into providing sustainable Internet access for remote and rural broadband continues but is still constrained by limitations such as unreliable power systems (Hasan, Barela, Johnson, Brewer, \& Heimerl, 2019).

The literature review led to some early assumptions regarding what functionalities potential MVPs may have, before the Grounded Theory study was carried out. Across nearly all research that includes remote and rural broadband access, off-grid power systems are an area 
of concern. No matter if rural broadband is provided over unlicensed $5 \mathrm{GHz}$ spectrum, or contested for through multimillion-dollar spectrum auctions, devices that are not connected to a regional power grid must generally be powered through renewable energy sources such as solar or wind. Upon looking deeper into off-grid power related issues, it was found that many small providers struggle to justify over-provisioning solar power systems, due to the high cost of solar panels and the necessary batteries for keeping sites online for extended periods.

As a result, small WISPs that have under-provisioned power systems are often susceptible to downtime during stormy periods (Surana et al., 2008). Aside from unreliable off-grid power systems, the last decade has seen researchers from across the world identify a series of issues regarding the provision of broadband access in remote and rural communities. While not always in the context of WISPs, challenges span from poor operational management through to poor network performance, and difficulties initially establishing networks (Pötsch, Yousaf, Raghavan, \& Chen, 2018). As would be expected, each area of challenge warrants its own demand for further research. Unfortunately for those living in remote and rural communities, last-mile connectivity is still an area where sustained research is necessary across a variety of research problems.

Despite an abundance of research broadly related to wireless networks, little has been done to encourage the long-term sustainability of WISP networks. This is likely due to issues identified in university environments differing to those experienced first-hand by WISPs serving isolated communities. However, limited literature did not constrain the Grounded Theory study that was conducted. In Grounded Theory, preconceived ideas are intended to be kept to a minimum, meaning an initial literature review is not necessary (Glaser \& Holton, 2004). The reduction of preconceived ideas also meant that initial product development assumptions had to be kept deliberately vague. The interview questions used for the study are available in Appendix 2 - Interview Questions. 
Based on the brief literature review, and Grounded Theory being chosen as a research methodology, a set of broad project development assumptions were drafted before carrying out data collection:

1. Many WISP operators struggle to scale their networks and build robust Internet infrastructure.

a. This assumption is based on the preliminary findings from the literature review, and observations from the researcher. In the case of Venture Networks, the primary Heights Road site came into possession of the company following the collapse of another WISP in the area. In the Horowhenua, the researcher knows of at least two WISPs that have either collapsed or had network assets acquired by other providers due to financial struggles.

2. Low-budgets and regional financial constraints are crucial issues for ubiquitous broadband access.

a. This assumption is based on observations of the researcher. Even in developed nations where fast Internet infrastructure exists, financing WISP networks is a barrier to their expansion. In New Zealand, several WISPs nationwide have benefited from the New Zealand Government's RBI1/RBI2 programmes. However, in other regions of the world, external, debt-free funding may not be available, meaning a business case for the WISP must be made. Unfortunately for many WISPs, broadband infrastructure in remote and rural communities will likely have a very slow return on investment. This is typically due to sparse population densities, and low regional household incomes, making WISPs unappealing to potential sources of funding.

3. Well established, large service providers may struggle to see the value proposition of low barrier to entry products.

a. For well-resourced Internet Service Providers (ISPs), low-cost, easy-to-use platforms may have little appeal. ISPs typically have large teams that can perform complex planning, network configurations, and ongoing support, as necessary. In the case of WISPs, the operational tasks described are likely 
carried out by a small team, or even a single person. It should be clear that the target of the research is small regional operators without the human and financial resources of their large counterparts.

4. MVP prototypes will be developed and tested before the business case is finalised.

a. This report tentatively describes an MVP and the best business model to be used for the development and ongoing commercialisation of it. It should be recognised that once the proposed real-world tests of the MVP are completed, the business model later described may pivot, as necessary.

5. A substantial amount of initial $\mathrm{R} \& \mathrm{D}$ can be completed internally without external funding.

a. Venture Networks aims to complete the initial development of the MVP without the need for external funding. This is to demonstrate to potential funding sources that a large-scale feasibility study of the MVP is viable to further test its commercialisation potential.

A total of 34 in-depth interviews were carried out to complete the Grounded Theory study. The initial cohort of interview participants consisted of WISPs from New Zealand. Before divulging the full results of the Grounded Theory study, preliminary findings from the New Zealand WISPs uncovered areas of continual concern: 
Aligned with findings from the initial literature review, solar power systems were identified as one of the top two challenges faced by WISPs in New Zealand. Upon discussing power systems with WISPs in depth, it was discovered that the cost of solar power systems prohibits the deployment of higher speed backhauls. In some cases, this prevented WISPs from futureproofing network sites against increasing demand for traffic. Furthermore, radio spectrum was found to be the most frequently recurring challenge area for New Zealand WISPs.

Compared to cellular providers that operate using expensive spectrum contested for through multimillion-dollar auctions, WISPs typically use unlicensed spectrum available without fee through Radio Spectrum Management (RSM), which is a division of the Ministry of Business, Innovation and Employment (MBIE).

A summary of the radio spectrum available for unlicensed use in New Zealand is shown below:

\begin{tabular}{|l|l|}
\hline 900MHz Wireless GURL & \\
\hline $915 \mathrm{MHz}$ to $920 \mathrm{MHz}$ & 1 watt $(30 \mathrm{dBm})$ total EIRP \\
\hline $920 \mathrm{MHz}$ to $928 \mathrm{MHz}$ & 4 watts $(36 \mathrm{dBm})$ total EIRP \\
\hline $\mathbf{2 . 4} \mathbf{~ G H z}$ Wireless GURL & \\
\hline 2400 to $2483.5 \mathrm{MHz}$ & 4 watts $(36 \mathrm{dBm})$ total EIRP \\
\hline
\end{tabular}




\begin{tabular}{|l|l|}
\hline $\mathbf{5 ~ G H z}$ Wireless GURL & \\
\hline $5180 \mathrm{MHz}$ to $5250 \mathrm{MHz}$ & $200 \mathrm{~mW}(23 \mathrm{dBm})$ total EIRP (indoor only) \\
\hline $5250 \mathrm{MHz}$ to $5350 \mathrm{MHz}$ & $200 \mathrm{~mW}(23 \mathrm{dBm})$ total EIRP (indoor only) OR 1 watt (30 dBm) \\
total EIRP for outdoor use (DFS enabled)
\end{tabular}

\section{Table 11 - New Zealand public radio spectrum allocations.}

For WISPs, the following two public spectrum allocations are critical:

\begin{tabular}{|l|l|}
\hline $5725 \mathrm{MHz}$ to $5850 \mathrm{MHz}$ & 200 watts $(53 \mathrm{dBm})$ total EIRP (point to point only) \\
\hline $5725 \mathrm{MHz}$ to $5875 \mathrm{MHz}$ & 4 watts $(36 \mathrm{dBm})$ total EIRP (point to multipoint only) \\
\hline
\end{tabular}

\section{Table 12 - Spectrum typically used by WISPs in New Zealand.}

It is important to note that New Zealand WISPs can only use a legal maximum EIRP of 4 watts for point to multipoint $5 \mathrm{GHz}$ links. As EIRP is the product of radio output power and antenna gain, WISPs are constrained to relatively short-range point to multipoint networks. The 200 watts EIRP for point-to-point communications enables long-range $5 \mathrm{GHz}$ backhauls to be established, potentially over tens of kilometres. The result of these regulations is that WISPs who want to provide wide-spanning coverage must either build many small network sites, use comparatively expensive licensed radio gear, or illegally use higher output EIRPs for point to multipoint communications.

The other challenges identified across the New Zealand WISPs are wide-ranging and require further investigation to thoroughly understand. To avoid designing a product around requirements specific to only New Zealand WISPs, further interviews were conducted with 
international participants, including WISPs, networking consultants, and academics.

Following the completion of in-depth interviews, Grounded Theory started to emerge. It was discovered that:

Start-up and established WISPs regularly lack the technical know-how to design and build highperformance, scalable networks from the outset. Consequently, network resources are not utilised to their full potential, and network performance suffers. This ultimately results in networks that cannot scale due to technical and operational inadequacies.

To support the emerging theory, months of discussions from two key WISP-oriented social media groups were analysed. A comparison diagram showing the overlap in discussions between the two groups is shown. The middle three nodes show the overlapping categories: 
The overlapping categories shown in the illustration above support the Grounded Theory developed, and show that designing WISP networks, selecting the most appropriate hardware, and general operational issues are areas of concern for WISPs worldwide. When the results of social media analysis were aggregated with interview findings, it was found the top challenge areas, or customer pains experienced by WISPs, are directly related to:

- Network design.

- Finance.

- Network hardware (routing and switching). 
To better explain why these categories are of interest, a needs analysis map was created. The map shows the needs of WISP operators and visualises what features must be considered for use in the MVP.

\subsection{1 - MVP feature requirements}

Following the development of Grounded Theory, findings were discussed with key research stakeholders. The discussions formed differing opinions, with some disagreement between stakeholders as to what the best solution based on the requirements should be. Key to designing the MVP was abstracting the Grounded Theory developed, and the product requirements identified in the needs analysis map, into actual MVP features. Early consultations with the research Advisory Board suggested the development of a software 
solution would be reasonable, as opposed to developing hardware, due to the higher-resource requirements needed for reiterative hardware development.

After deliberating possible solutions, the idea of designing a low-cost Software-Defined Networking platform was put forward by the researcher. The concept of developing an SDNbased MVP enables many of the higher-level requirements of WISPs to be realised in actual product functionality. SDN typically involves decoupling the control and data planes of a network and utilising a central controller to perform network forwarding decisions. The functionality of the proposed MVP is no exception. However, some distinct features were conceptualised to ensure performance in a wireless context where controller availability may be unpredictable.

Following the conclusion of discussions with stakeholders, a set of technical requirements for the MVP was planned, derived from the challenge areas, or customer pains, identified:

\begin{tabular}{|c|c|}
\hline Challenge area and market needs & Platform integration \\
\hline $\begin{array}{l}\text { Network hardware (routing and } \\
\text { switching): } \\
\text { - } \quad \text { Easy customer management. } \\
\text { - } \quad \text { Simple configuration. } \\
\text { - } \text { DC input, PoE output. } \\
\text { - } \quad \text { Robust off-grid power } \\
\text { - } \text { management. } \\
\text { - } \quad \text { Low cost. } \\
\text { - Stable firmware. }\end{array}$ & $\begin{array}{l}\text { Existing commercial routing/switching platforms not } \\
\text { suitable for modification -> new platform needed. } \\
\text { - The platform must accept a wide range of DC input } \\
\text { voltages, have programmability to manipulate network } \\
\text { devices based on battery bank voltage. } \\
\text { - The platform must be waterproof, able to survive } \\
\text { extremely hot and cold environments. } \\
\text { Platform software must be stable as it will be } \\
\text { responsible for mission-critical network systems. } \\
\text { Keeping costs low to maintain a competitive } \\
\text { advantage must be considered throughout } \\
\text { development. }\end{array}$ \\
\hline $\begin{array}{l}\text { Finance: } \\
\begin{array}{l}\text { - Certainty of a return on } \\
\text { investment. } \\
\text { - Predictable network operations. }\end{array}\end{array}$ & $\begin{array}{l}\text { Finance is a crucial area of challenge for WISPs } \\
\text { worldwide; to be considered by WISPs, especially } \\
\text { those just starting the platform developed must prove } \\
\text { it can perform at a reasonable cost. }\end{array}$ \\
\hline
\end{tabular}




\begin{tabular}{|c|c|c|}
\hline - $\quad$ Inv & & $\begin{array}{l}\text { Platform emphasis on quality and robustness - } \\
\text { stringent testing must be carried out before mass- } \\
\text { market production. }\end{array}$ \\
\hline $\begin{array}{l}\text { Network design: } \\
\text { - } \text { Effective use of spectrum. } \\
\text { - Utilises network resources. } \\
\text { horks with existing network } \\
\text { - } \text { Predictable operations. } \\
\text { - Quality of service and } \\
\text { performance. } \\
\text { - Low barrier to entry (little staff } \\
\text { knowledge and training } \\
\text { required). } \\
\text { Automation of manual processes } \\
\text { to reduce human interaction with } \\
\text { the network. }\end{array}$ & - & $\begin{array}{l}\text { The platform must effectively utilise all network } \\
\text { resources where possible. } \\
\text { The platform should incorporate the "hard to } \\
\text { implement protocols" such as MPLS, OSPF, in an } \\
\text { easy-to-use manner. } \\
\text { The platform should have a low barrier to entry, so } \\
\text { that little prior knowledge is required. } \\
\text { The platform must work with existing wireless radios } \\
\text { from a range of vendors, e.g., Ubiquiti, Mimosa, } \\
\text { Mikrotik. } \\
\text { The platform must have a clear advantage over } \\
\text { existing products on the market, in terms of ease of } \\
\text { use, and functionality. } \\
\text { As well as equal cost forwarding, traffic should be able } \\
\text { to be steered depending on higher-level business } \\
\text { requirements, specified by the network owner/WISP. }\end{array}$ \\
\hline
\end{tabular}

Table 13 - Market needs and platform integration.

A high-level abstraction of the components used for the proposed MVP is shown: 
Initial testing of the proposed MVP was completed using Mininet, which is an open-source network emulator designed for experimenting with OpenFlow capable networks. Following successful testing of the MVP using Mininet, identifying an appropriate low-cost hardware platform was also necessary. The table below describes the components shown in the abstraction above and how they have been implemented to date:

\begin{tabular}{|c|c|}
\hline Component & Implementation \\
\hline SDN controller & $\begin{array}{l}\text { - Venture Networks has implemented a modified version of the OpenMul } \\
\text { SDN controller, written in the C programming language for efficiency and } \\
\text { speed. } \\
\text { For testing, the SDN controller has been implemented on a virtual } \\
\text { machine and tested within a Mininet environment. } \\
\text { - For real-world use, the SDN controller can be extended to run on nearly } \\
\text { any Linux based system. } \\
\text { Users can operate the controller on any platform of their choice. } \\
\text { However, Venture Networks will supply a quad-core, single board } \\
\text { computer complete with } 4 \text { Intel Gigabit Ethernet adapters and } 4 \mathrm{~GB} \text { of } \\
\text { ram for } \$ 350 \text { NZD. }\end{array}$ \\
\hline
\end{tabular}




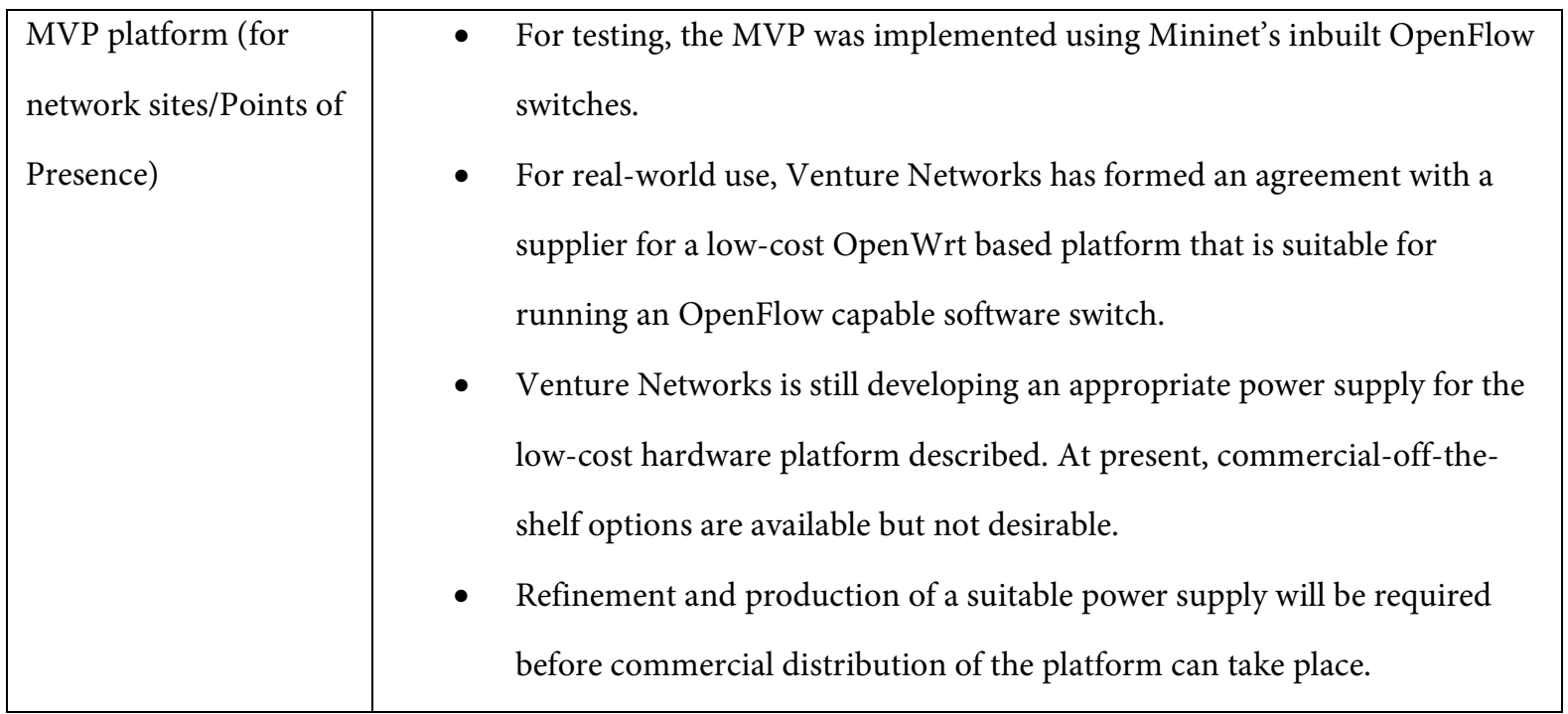

Table 14 - MVP Implementation.

\subsection{2 - MVP testing}

As briefly mentioned, Mininet was utilised as an appropriate platform for initially testing the proposed MVP. Venture Networks has completed a significant amount of development of the platform, primarily using Mininet for testing generic WISP network topologies. To date, the MVP has performed well in tests and is nearing readiness for deployment on a physical network. Venture Networks prioritised functionality over user-friendliness during the development process. Before further physical tests are completed, Venture Networks wishes to refine the user interface of the system and make it as straightforward as possible to use. Finally, Venture Networks has been in contact with WISPs interviewed earlier in the study; several of which are interested in working with Venture Networks to test the proposed MVP.

So far, simulations of the MVP in Mininet have shown the SDN controller to be capable of handling 128 OpenFlow capable switches without performance degradation. In most SDN environments, every time a new network flow is detected, it is sent by the OpenFlow switch to the controller, which then instructs the switch what to do with the flow. In a wireless network, where latency is unlikely to be predictable, especially across tens of network nodes (or OpenFlow switches in this case), the time taken for the installation of a flow into a switch 
would introduce an unaffordable delay. To mitigate this, and for other purposes, the MVP uses an overlay network.

In the case of the MVP, Virtual Extensible LAN (VXLAN) is used to provide this overlay, although many other methods of encapsulation could be used, such as L2TP or even GRE tunnels. Traffic forwarding decisions are made proactively by the controller, and flow installations are only performed on IP addresses that are part of the underlay network. The result is fewer flow installations existing on each switch, and latency only being introduced to the network in the event a link goes down, and the controller must compute a new path. In many cases, even when a link goes down, there will be no noticeable latency introduced for end-users unless all the customer's traffic must be sent over a different link. This is due to Equal Cost Multipath forwarding (ECMP) being performed by the OpenFlow switches.

Finally, the benefit of having an overlay network means that WISP operators no longer must worry about (potentially) complex layer-3 routing configurations. This means that WISPs no longer need to concern themselves with how Interior Gateway Protocols (IGPs) such as OSPF should be configured. A WISP using the MVP can set it up as they would a simple layer-2 switched network, while experiencing the benefits seen in other networks established by experienced network engineers. Utilising underlay and overlay networks also mean that WISPs can have effortless, centralised control over what IP addresses are allocated to customers. As a result, WISPs can provision different services based on business requirements, without the hindrance of complex technical configurations.

The low-cost, OpenWrt based hardware platform Venture Networks has selected for use is also capable of far more than is currently required, making it future-proof and simple to extend should it be necessary in the future. To further reduce the cost of the MVP, Venture Networks is also considering porting an OpenWrt compatible version of the SDN controller. An OpenWrt compatible version of the SDN controller will further reduce the expenses of the MVP passed on to WISPs. The OpenWrt based platform Venture Networks has chosen for 
development is inexpensive compared to many other Linux based systems, costing around $\$ 30$ NZD. This price is excluding the cost of the off-grid power supply that is also needed, which is expected to cost a similar amount when mass-produced. A photo of the (non-PoE enabled) OpenWrt platform used for the proposed MVP is shown below:

Other than technical performance testing, the MVP will require user experience testing to be conducted. It has been established that WISPs often struggle with the technical implementation of their networks. To target these WISPs, Venture Networks will need to offer consultancy services, and promote the MVP to those with inadequate networking expertise. To gain invaluable feedback before a final MVP is released, Venture Networks will provide select WISPs with the MVP in a pre-release form for testing. Feedback from these WISP operators will then be incorporated into the platform as deemed necessary prior to final feasibility testing and subsequent product release.

\subsection{3 - MVP development and manufacturing}

Software development and modification of the OpenMul controller is being carried out at Venture Networks' Levin office. Manufacturing of the low-cost OpenWrt platform will be achieved using contract manufacturing with a Chinese supplier. Despite outsourced 
manufacturing, Venture Networks intends to keep research and design within the Horowhenua district, to encourage the growth of jobs and to retain income within New Zealand. Venture Networks also plans on assembling the first batch of experimental power supplies at their Levin workshop. Pending the successful trial of the experimental power supply units, Venture Networks will seek another contract manufacturer for the production, and likely assembly of the units.

Venture Networks has already ordered a sample batch of the low-cost OpenWrt platform through the contract manufacturer. Features of the OpenWrt platform include:

- Dual-core $880 \mathrm{MHz}$ CPU (MediaTek MT7621A).

- $256 \mathrm{MB}$ of DDR3 RAM.

- $16 \mathrm{MB}$ of SPI flash storage.

- 5 gigabit Ethernet ports, with a CPU integrated 5 port gigabit switch.

- $<5$ watts power consumption.

These boards are supplied at approximately $\$ 30$ NZD per unit, a price that will be further reduced through high-volume orders. Unfortunately, the cost to manufacture the platform in New Zealand is prohibitively high at this stage. It is in favour of Venture Networks to work with a contract manufacturer that is already well established and has a stable supply of CPUs sourced from MediaTek. Venture Networks would otherwise need to acquire manufacturing equipment, further increasing expenses. Even if Venture Networks were to have bare Printed Circuit Boards (PCBs) manufactured through a third party, they would still need to acquire several key machines for high-quality MVP assembly to take place in Levin:

- Reflow oven.

- Solder paste printer.

- Pick and Place machine.

- Automatic Optical Inspection (AOI) machine.

- Transport conveyors. 
(Olimex, 2017).

Assuming new hardware from reputable brands, the initial expense for the necessary manufacturing equipment would in excess of $\$ 100,000$ NZD, and require additional staff and training.

\subsection{4 - Intellectual Property protection}

The modified OpenMul controller is licensed under the GNU General Public License v2.0. A summary of the GPL v2.0 license is detailed below:

\section{Permissions}

- Commercial use is okay.

- Distribution is okay.

- Modification is okay.

- Private use is okay.

\section{Conditions}

- Software source must be released upon distribution or commercialisation of the product.

- A copy of the GPL v2.0 license must be included with the software.

- The same license must be used upon distribution.

- Changes to the original code must be documented.

\section{Limitations}

- The GPL v2.0 license includes a limitation of liability.

- The GPL v2.0 license explicitly states it provides NO warranty.

Venture Networks was aware before the start of development that the OpenMul controller is licensed using the GPL v2.0 license. However, Venture Networks believes releasing the modified controller as open source will encourage other organisations and researchers to sustain the development of it in the long-term, should a critical mass of WISPs start to use the 
MVP. Other components of the MVP have involved original development, and Venture Networks is actively pursuing the possibility of applying for patents where possible.

\subsection{5 - Improved coverage zone}

Venture Networks proposes carrying out a practical feasibility study of the MVP. While the simulated performance of the MVP is promising, Venture Networks believes that significant economic benefits can be realised through practical deployment and testing of the platform. Venture Networks already has a commercial Internet access network operating across much of the Horowhenua, creating an ideal testbed for establishing whether the platform is feasible for deployment on a real WISP network. In addition to deploying the MVP on existing network sites, Venture Networks believes that a feasibility study will be greatly assisted through the creation of new sites, which will immediately benefit rural residents across the Horowhenua district.

Coverage of the existing network spans approximately 500 square kilometres. The propagation map below shows this coverage and excludes small sites across the region: 
Under the proposed expansion, additional network sites would be established. Pending land access agreements being formed with property owners, Venture Networks seeks to establish new network sites at:

- Heights Road (power upgrades to the existing site).

- Heights Road (new site, lower elevation area of the Heights Road for coverage of

Shannon and surrounding areas).

○ Up to 360 square kilometres of new coverage.

- Tokomaru (new site)

- Up to 390 square kilometres of new coverage.

- Moutere Hill (new relay site).

$\circ$ Point to point coverage for nearby sites and later

- Hokio Sands Road (new site).

○ Up to 90 square kilometres of new coverage. 
- Waitarere Beach (public WiFi access point from the local 4Square and Surf club).

- These sites are not initially intended for household Internet access.

- Ohau (new site).

○ Up to 55 square kilometres of new coverage.

- Motuiti and Paranui Marae (new sites).

○ Free WiFi hotspots for use at both Marae.

Venture Networks has tentatively offered both Motuiti and Paranui Marae free Internet access as a part of the proposed network upgrades. Both Motuiti and Paranui currently do not have Internet access and would be direct beneficiaries should the upgrades be completed. Digital connectivity would be beneficial for Ngati Raukawa, the iwi affiliated with both Marae, as holding meetings with external stakeholders could be carried out over the Internet. The upgrades would also enable those living near both Marae without Internet access to experience the full benefits of fast Internet connectivity. Connectivity to both Marae will be accomplished using a new dedicated backhaul link from either the existing Heights Road site, or from the proposed Tokomaru site. Motuiti and Paranui Marae (located just north of Foxton) are shown on the map below: 
The first propagation map shows a key displaying signal strength received in $\mathrm{dBm}$ (decibelmilliwatts), a measure of received power. In practice, signal strengths received by remote devices (clients) on WISP networks that range from $-80 \mathrm{dBm}$ to $-90 \mathrm{dBm}$ are not desirable and should be avoided unless in an emergency. Signal strength worse than $-90 \mathrm{dBm}$ is not 
usable. When interpreting the graph, a substantial amount is represented as being approximately $-90 \mathrm{dBm}$. In practice, Venture Networks has found the received signal strength at most client locations across the Horowhenua to be superior, likely due to the little interference from existing devices.

Despite performance currently being at an acceptable level, the $5 \mathrm{GHz}$ spectrum is not licensed and has no legal protection against interference from other providers. Due to the possibility of future interference and poor network performance, Venture Networks, and WISPs in general, must plan future network expansions with great care. To keep ahead of the demand for bandwidth, Venture Networks has observed that some WISPs are pivoting their businesses and utilising Fibre to the Home (FTTH) deployments to provide ultra-fast services to their customers. However, WISPs turning into FTTH providers are uncommon in New Zealand due to the UFB programme. For WISPs that do deploy FTTH networks, capital and operational expenses are high, typically with slow returns on investment.

The upfront costs of buying fibre optic cable and the required networking equipment to provide services over it are relatively low. For fibre optic deployments, the costs of deployment come from more than just the cable and provisioning equipment. Fibre optic cables are typically trenched or, less frequently in New Zealand, installed overhead alongside power lines. The process of trenching cables is inherently expensive, as it requires the purchase or hire of excavating equipment, traffic control, and staff to facilitate operations. Overhead installation is similarly expensive, as specialist line workers are required to install fibre optic cabling alongside high-voltage power lines. Additionally, utilising overhead lines will likely incur a cost from the lines network, further reducing the feasibility of deployment.

Given the expensive nature of deploying fibre optics, other innovative means of provisioning Internet connectivity are needed. Venture Networks proposes the creation of a separate segment of the network to be devoted to testing the MVP in a different environment - a millimetre wave (mmWave) $60 \mathrm{GHz}$ mesh topology. The $57-60 \mathrm{GHz}$ spectrum can be utilised 
in New Zealand, license free, and can provide massive throughput to devices that utilise it. However, mmWave network devices suffer from environmental limitations such as atmospheric oxygen absorption and rain fade (Singh et al., 2018). Consequently, mmWave networks are only useful at short-range. Despite challenges with range, point to multipoint mmWave radios are now commercially available at sub-\$200 NZD prices, making them an appealing option for creating ultra-fast, short-range mesh networks.

Venture Networks is currently surveying appropriate areas to construct the $60 \mathrm{GHz}$ network segment. For maximum impact, Venture Networks wishes to locate an area that has a relatively high population density, so that the short-range $60 \mathrm{GHz}$ equipment is financially viable. Additionally, the Ultra-Fast Broadband $2 / 2+$ expansions mean that areas in the Horowhenua such as Waitarere Beach, Hokio Beach, Ohau, and Tokomaru will all be fibreconnected by the end of 2022 (Toury, 2019). With the expansion of UFB2/2+ in mind, Venture Networks needs to seek out small communities that will not receive connectivity upgrades as part of the UFB2/2+ schemes, such as Tangimoana. Secondly, rural lifestyle block developments are an ideal target, as deployment costs of a mmWave network in comparison to a fibre optic network will greatly favour mmWave.

Developing a mmWave mesh network will enable the MVP to be tested with rigour. The proposed site upgrades and additions will enable the performance of the MVP to be evaluated in comparison to traditional routing protocols used by WISPs, which are typically a combination of OSPF, MPLS and sometimes BGP. However, the addition of short-range mmWave radios means far more devices operating on the network, and an opportunity to measure the real-world performance of the MVP. Notably, performance metrics of the MVPs controller are of interest, including the measurement of convergence times in case of a network site failure. Presenting the results of real-world, practical tests is itself a marketing instrument that Venture Networks wishes to utilise when promoting the MVP. 


\subsection{6 - Project development timeline}

The timeline below shows an overview of the proposed MVP development across the next year. It is also indicative of the proposed network expansion should Venture Networks secure funding for a feasibility study of the MVP to be completed:

\subsection{7 - Business model design}

For its existing business operations, Venture Networks follows a service provider business model. Users of the network pay a predictable, $\$ 69$ per month fee. As Venture Networks wishes to expand its business operations, this model must also evolve. After discussing 
appropriate business models with the project Advisory Board and the other Directors of Venture Networks, it was agreed that a unique business model is needed to facilitate the company moving forward. The business models of two other telecommunications companies, Ubiquiti Networks and Netonix, were examined. Both Ubiquiti and Netonix have unique business models that are not common in other markets.

For both companies, working directly with end-users (typically being WISP operators or those wishing to become WISP operators) has proven invaluable for building a community of loyal supporters. No sales team exists for either company, and marketing is performed by word of mouth through service providers, resellers, distributors, and other IT professionals. Engineers interact with customers directly over company forums and social media. Customers are drawn to using each company's products as both are responsive to user feedback, and development periods tend to be fast compared to competitors. Ubiquiti also has an advantage with product and cost leadership. Compared to their competitors, who have mostly stagnated, Ubiquiti has dominated the WISP market and is now gaining attention in the corporate networking market.

The development of an appropriate business model for Venture Networks and the MVP was completed using the Business Model Canvas (BMC) of Strategyzer AG. The BMC is a tool that enables a universally recognisable format for what a business model is (Osterwalder, 2010). In collaboration with Venture Networks, a BMC was created based on the findings of the Grounded Theory study and iteratively updated to meet evolving requirements. The MVP was also developed using the BMC. Kromer (2014) identified that to create an MVP, four of the BMC components can be used:

5. Customer Segments.

6. Value Propositions.

7. Channels.

8. Customer Relationships. 
An overview of the proposed MVP derived from the four components discussed is shown in the table below:

\begin{tabular}{|c|c|}
\hline $\begin{array}{l}\text { Customer Segments - Who are our most } \\
\text { important users? }\end{array}$ & $\begin{array}{l}\text { Start-up WISPs, particularly ones that are uncertain about how } \\
\text { to design their networks. }\end{array}$ \\
\hline $\begin{array}{l}\text { Value Propositions - Which challenges } \\
\text { do we hope to solve? }\end{array}$ & $\begin{array}{l}\text { We aim to alleviate the struggle that WISPs have when it comes } \\
\text { to designing robust, performant networks. The ability to } \\
\text { operate robust WISP networks extends to what is potentially } \\
\text { significant cost-saving through lower staff training } \\
\text { requirements, less network downtime, and less dependence on } \\
\text { external assistance (consultants). }\end{array}$ \\
\hline $\begin{array}{l}\text { Channels - How are we reaching our } \\
\text { Customer Segments? }\end{array}$ & $\begin{array}{l}\text { Customer Segments targeted will primarily be reached through } \\
\text { social media platforms such as Facebook, where help groups } \\
\text { such as Wisp Talk already exist and have a well-established user } \\
\text { base. }\end{array}$ \\
\hline $\begin{array}{l}\text { Customer Relationships - What } \\
\text { relationships do we have established, what } \\
\text { relationships are needed, and what is } \\
\text { expected of us? }\end{array}$ & $\begin{array}{l}\text { The researcher already has well-established relationships with } \\
\text { several New Zealand WISPs. However, New Zealand WISPs } \\
\text { interviewed are well established, suggesting we should } \\
\text { approach these relationships later, once a product has been } \\
\text { developed. } \\
\text { WISP start-ups that participated in interviews will be } \\
\text { approached again through social media for the sake of MVP } \\
\text { development and testing. Meeting with (where possible) and } \\
\text { working with these WISPs will be essential for gauging how } \\
\text { effective the product is and pivoting, as necessary. }\end{array}$ \\
\hline
\end{tabular}

\section{Table 16 - MVP overview.}

Despite the MVP being implicitly referred to as a tangible product throughout this expansion plan, it consists of several technical components listed in the table above (customer segments, value propositions, channels, and customer relationships). The full business model proposed for Venture Networks is shown below: 


\subsection{8 - Limitations}

Like any product, the MVP does have some technical limitations. Venture Networks acknowledges that using the Software-Defined Networking paradigm for WISP networks introduces some challenges. Each challenge area identified during development is described below:

\section{Controller latency}

In Software-Defined Networking, a centralised controller is typically used to install rules into network switches, telling them where to forward traffic flows. Unless rules are proactively installed, new flows on the network introduce latency, as a switch must send a packet_in message to the controller. The controller will then install an appropriate path for the flow to take. In an environment where link speeds are predictable, and latency is low, a fast controller 
could process tens of thousands of flow installations every second. In the case of the OpenMul controller used for the MVP, benchmarks show that with 128 switches connected, approximately 2.6 million flows per second can be processed, with average flow setup time taking around $10 \mu$ s (Sonba \& Abdalkreim, 2014). However, it is yet to be discovered how flow-installation latency introduced by the controller will perform in a real-world WISP network; hence the necessity for the feasibility study proposed.

\section{Controller failure}

As opposed to decentralised link-state routing protocols such as OSPF which are typically used in WISP networks, the MVP requires centralised control. In the case of a controller failure, network switches cannot work autonomously, and the network will cease to function. For the MVP to function successfully, the software quality must be kept to an exceptionally high standard. To mitigate against critical network failure, additional controllers can be placed on the network and act as slaves to the master controller. However, in a WISP network with low development budgets, an extra controller and high availability of network devices may be an afterthought until it is too late, and failure has already occurred.

\section{Loop avoidance and broadcast handling}

Another issue that plagues switched networks is how layer-2 loop avoidance is handled. It is good practice to avoid building large scale switched networks, for a range of reasons. In switched networks where layer-2 loops exist, typically either the Spanning Tree Protocol (STP) or Rapid Spanning Tree Protocol (RSTP), which has faster convergence times, is used. However, utilising STP or RSTP means that ports that are causing loops on switches are logically disabled. In the case of WISP networks, this means that precious network resources cannot be utilised. An illustration showing how STP works in a network is shown below: 
The two links from switches 4 and 5 to switch 7 are logically disabled, to prevent a layer- 2 loop that would otherwise exist. The simple illustration shows a significant limitation of STP and RSTP - the best paths in a network are not guaranteed. If the topology above showed routers running an Interior Gateway Protocol (IGP) such as OSPF, the best path for data from router 2 to router 7 could be sent over Equal Cost Multipath (ECMP) paths using routers 4 and 5 . In the case of the MVP, broadcast traffic is sent over the same path as would be computed by STP in the illustration, while allowing traffic to be sent over ECMP paths using switches 4 and 5 to switch 7. Additionally, the MVP controller can act as an ARP proxy, reducing the need for unnecessary ARP broadcasts on the network.

\section{Convergence times}

In the case of network site failure on a WISP network, the controller component of the MVP is responsible for calculating the new path(s) for data to be sent. Fast-failover groups are installed on the switches, meaning that if an adjacent site were to fail, the traffic is automatically routed over other available paths without having to be reconfigured by the 
controller. Resultantly, there is no noticeable latency introduced. Despite testing to confirm the fast-failover functionality works as expected, the feasibility study will enable Venture Networks to evaluate how the MVP performs in comparison to traditional routing protocols found in WISP networks, i.e., typically OSPF. The performance metrics gathered will also enable comparisons to be drawn to mesh networking protocols typically found in ad-hoc wireless networks, such as the Optimized Link State Routing Protocol (OLSR) and the Better Approach To Mobile Adhoc Networking (B.A.T.M.A.N.) protocol.

\section{Power supply limitations}

Finally, the power supply unit being developed is currently a limitation to the MVP. Wireless radios that WISPs use range vastly, as do their power sourcing requirements. Most wireless radios are powered using Power over Ethernet (PoE); however, not all manufacturers adhere to the IEEE set of PoE standards. As a result, some manufacturers use what is referred to as passive PoE, where power from the sourcing equipment is always enabled, and not negotiated through a separate PoE controller circuit as it would be when compliant to IEEE 802.3af and IEEE 802.3at. As a result, some radios do not adhere to any IEEE standard and use either passive $24 \mathrm{v}$ or $48 \mathrm{v}$ power inputs. Other manufacturers will use the IEEE standards, which are typically not compatible with non-compliant passive systems. As a result, Venture Networks must consider which options should come as standard on the MVP, while maintaining affordability of the platform.

Due to complaints from New Zealand WISP operators regarding the expensive nature of solar power systems locally, Venture Networks believes that future research into making the MVP energy aware is necessary. Future research will focus on how the MVP can take the capacity of 'green energy', i.e., solar and wind systems, into consideration while operating. Making the MVP energy aware would enable far better resource utilisation than what is currently implemented. Following the completion of the feasibility study, Venture Networks believes pursuing energy aware networking to be a worthy cause - and one of particular interest to WISP operators in New Zealand. 


\section{5 - Market validation}

The study conducted, and the development of Grounded Theory, served several purposes:

- To understand WISP operators and their needs.

- To determine what MVP would be appropriate to develop.

- To validate there is a market need for the MVP to be developed.

- To validate project development assumptions.

For market validation, Grounded Theory provided invaluable insights into how WISPs come about, and how they go about building infrastructure in remote and rural communities. Most of the WISPs interviewed starting building infrastructure out of frustration with slow alternatives and their high prices.

While many WISPs interviewed had already been operating for some time, it is not to say that future customers will be well-established. As WISPs often start out of necessity, they are not always well resourced financially or technically. For Venture Networks, capturing the attention of frustrated individuals with an average technical ability will be crucial. Additionally, the in-depth interviews showed that even for WISPs that are well established, they are still not necessarily well resourced. Their staff tend to lack intricate knowledge of how to establish high-performance and robust WISP networks, something that the MVP aims to improve upon.

Ideal customers for Venture Networks are not bound by geography. The remote and rural telecommunications market is global and expanding as the Internet becomes an even more integral part of life outside of urban areas. For those living in rural communities where little infrastructure investment exists, the MVP offers a user-friendly way of building robust networks without the need for expensive external help. Essentially, Venture Networks aims to enable rural networks to be built and run by their users, optimising what (likely limited) local resources exist to empower communities to develop infrastructure themselves. Thanks to 
Ubiquiti, high-performance wireless radios that are user-friendly and accessible to those without radio engineering knowledge exist. Venture Networks believes now is the time to disrupt routing and switching platforms to be just as user-friendly.

\subsection{1 - Changing behaviours}

During the development of Grounded Theory, the researcher observed prospective WISP operators taking to WISP-oriented social media groups for assistance getting started. Typically, most users will receive assistance in some form from experienced group members. The groups are also a market opportunity for consultants, who regularly use social media platforms for finding prospective clients. The best way to describe how Venture Networks believes market behaviours can be changed is through a real-world example. One such example is someone who was interviewed by the researcher, and later passively helped determine appropriate value propositions of the MVP. For the sake of anonymity, we will call this person Bob. Like many others, Bob was posting on social media groups in need of assistance:

\section{Who is the customer?}

Bob. Like many people across the world that live in remote and rural communities, Bob lacks Internet access at home and has limited mobile phone coverage. Without external funding through government-backed programmes, large telecommunications providers do not see the value or business case in building expensive infrastructure to serve such a small community such as where Bob lives. Bob is unhappy about not having Internet access and being left behind the rest of the world. Bob went around his neighbourhood looking for others that felt the same way. Along with his neighbours, Bob wants to build better Internet infrastructure and experience digital enrichment that can be realised through the Internet. 


\section{What are their problems?}

Just like many others who are subject to the 'digital divide', Bob does not know how he can go about improving Internet connectivity. Bob has no background in networking or telecommunications, and little theoretical knowledge on the subject. Despite lacking the necessary knowledge to build a robust network, Bob is a keen learner and willing to put in relentless effort to meet his goal of having Internet access. As described by another interviewee, "Bob is a picture of why we all started". Just as would be expected of Bob, the researcher identified that even when WISP networks grow to a substantial size, the operators often still struggle with technical implementation and network maintainability.

\section{How can these problems be solved?}

An estimated $45 \%$ of the world's population lives rurally (Pötsch et al., 2018). Of this vast group, it is not known how many suffer from poor or non-existent Internet connectivity. However, it became clear throughout the Grounded Theory study that potential customers like Bob are not uncommon. Despite gradual improvements, people like Bob are still underserved and represent precisely whom the MVP should benefit. For people who lack any strong technical background, but can still follow instructions, improving the design and usability of platforms requiring technical knowledge is essential. As a result, creating a userfriendly routing/switching platform for WISPs would be a sufficient value proposition, even without features that enable scalability and robust performance.

\section{Key features?}

To encourage the utilisation of local resources in the construction of WISP networks some key design considerations were adopted:

- Usability emphasis. Design the MVP platform to be user-friendly to the point where anyone with basic computer literacy can use it.

- Maintain the KISS principle (Keep It Stupid Simple). 
- Allow WISPs to create high-performance, robust networks without the need for external consultants or extensive experience.

- Zero-touch/hassle free configuration.

- Positioned as a next-generation platform for building WISP networks.

- Support easy interoperability with other vendors where possible.

○ The MVP should be extensible to work with other user-friendly WISP-centric platforms such as Ubiquiti's UCRM for billing and customer control.

- Keep pricing disruptive, so it is affordable to the target markets.

○ Users/Prospective WISPs in impoverished areas.

\subsection{2 - Market reaction}

Currently, prospective WISPs tend to turn to social media platforms when they are stuck, often resulting in them employing consultants for help. The customer pain identified here is being idle not knowing how to manage routing configurations, and subsequently relying on others for what is a fundamental part of operating a WISP network. The Grounded Theory study showed the significance of this issue. Routing and configuration related help posts are the most commonly recurring topic across all the social media groups analysed. While asking for help is undoubtedly a crucial part of learning, the extent of the elementary level networking questions being asked by WISP operators, established or not, is cause for concern. A WISP operator that lacks the fundamental understanding of their network will likely struggle with long-term growth and scalability, at least without extensive external help.

The process WISPs go through when surveying the best hardware to purchase is very much crowdsourced. Like anyone frugal, it was observed by the researcher that WISP operators regularly engage in discussions on social media platforms to discuss how well hardware is performing. For manufacturers, these posts can rapidly develop into situations where damage control is required when WISPs encounter unreliable software, or total hardware failure. Unsurprisingly, Venture Networks wishes to avoid becoming the target of such posts when 
issues inevitably occur. To help mitigate brand tarnishing, Venture Networks will need to form a community of product evangelists to support and promote the MVP.

As consultants are already responsible for alleviating the customer pain, i.e., WISPs not understanding how to correctly configure routing and switching hardware, having them onside will be necessary for the MVP developing a positive reputation. If consultants act as product evangelists, it will not only help them carry out their roles as consultants with greater ease, but also push potential customers towards buying products from Venture Networks as opposed to any other vendor. Venture Networks intends encouraging consultants to position the MVP as a next-generation platform that enables WISPs to avoid the headaches of manual network configurations while benefiting from disruptive prices and high-performance.

WISPs will still need assistance with other tasks, such as acquiring wholesale Internet connectivity, setting up a billing system, configuration and placement of radios, power systems planning, and so on. Unfortunately, no one solution will ever solve all these issues. The MVP can put a stop to WISPs from concerning themselves with the complicated routing configurations that must be followed across the network. Venture Networks believes the hardest challenge to changing market behaviours and persuading WISPs to utilise the MVP will be spreading positive awareness of the platform. Initially, spreading awareness may need to be done through time-consuming hands-on work with WISPs across the world, but should prove beneficial to Venture Networks in the long-term through strong customer relationships.

\subsection{3 - SWOT analysis}

To see how Venture Networks is positioned to complete commercialisation of the MVP, a brief SWOT analysis has been created: 


\section{Strengths}

- Venture Networks has evidence that there is a market demand for more straightforward to use routing/switching products, such as the MVP.

- Easier to use routing/switching products enables Venture Networks to target a broader market - WISPs with and without strong technical abilities.

- The MVP enables WISPs to better utilise network resources, abstract high-level business requirements into network forwarding policies.

- Venture Networks has a devoted team and is committed to working on remote and rural broadband connectivity for the long-term.

- Venture Networks is familiar with the challenges faced by WISPs first-hand.

\section{Weaknesses}

- Venture Networks lacks the research and development budget that market leaders such as Ubiquiti and Netonix have.

- Venture Networks lacks the brand and reputation that market leaders benefit from.

- Technical feasibility testing of the MVP in a practical manner requires an extensive network expansion for Venture Networks, or to directly work with another WISP that is willing.

\section{Opportunities}

- The MVP's target market is underserved.

- The MVP offers a unique selling point over alternative routing products available to WISPs, i.e., it allows difficult configurations to be performed by anyone.

- As people that live in remote and rural communities demand access to the Internet, new solutions for providing access must be found.

- Venture Networks has already developed positive working relationships with potential customers, such as 'Bob' mentioned earlier.

\section{Threats}

- A market leader such as Ubiquiti could bring a similar product to market before Venture Networks. 
- A market leader such as Ubiquiti could see value in the product and bring a competing product to market following the launch of Venture Networks MVP.

- Research and development can be expensive in New Zealand compared to having it outsourced.

- Software developed for the MVP has little margin for error.

\subsection{4 - Competing products}

No SDN-enabled products specific to WISP networks currently exist. However, enterprise SDN-enabled solutions are being championed by companies such as Ubiquiti Networks, through their UniFi product range. The UniFi range of products started as high-performance WiFi access points and evolved into a variety of products including switches, security gateways, cameras, VoIP phones, and even computer-controlled LED lighting. Building a variety of easy to deploy, manage, and maintain products has paid off for Ubiquiti, with the UniFi series realising $210 \%$ growth and greater than 10 million devices being shipped over the last three years (Ubiquiti Networks, 2017). Cambium Networks, a rival of Ubiquiti, has now launched their series of 'cloud-managed' switches, offering similar functionality to the Ubiquiti UniFi range, except without the SDN label being used for marketing.

\section{6 - Market research}

The global WISP market is estimated to be worth approximately USD 10 billion, as of 2013. The Total Addressable Market (TAM) is estimated at USD 1.4 billion, with Ubiquiti Networks accounting for USD 400 million of this as of 2017 (Ubiquiti Networks, 2017). Additionally, the annual market growth rate is estimated at 5-10\%. Venture Networks aims to capture a small portion of this market initially, and then branch into adjacent markets, as necessary. As the demand for better Internet connectivity increases in remote and rural communities, so must the way providers can establish a financially sustainable infrastructure. Venture Networks believes the MVP developed is innovative and can be easily deployed, 
empowering those with scarce resources to take ownership within their respective communities.

In a testament to the centralised control mechanism of the MVP, Ubiquiti Networks has developed a solution with similarities for the enterprise market. The Ubiquiti UniFi SDN controller enables easy deployment of home, office, and enterprise networks, all without having to perform individual configuration of switches and access points. The UniFi series of products has proven to be widely successful for Ubiquiti, generating almost half of their annual revenue, and accounting for approximately USD 400 million (Ubiquiti Networks, 2017). The promotion of SDN for the UniFi platform shows that as network innovation is evolving, so is the way ordinary people go about setting up networks.

While WISPs are generally not run by people with no technical experience, Venture Networks believes exploiting the path of least resistance is key to MVP success and marketability. Just as Ubiquiti has made their UniFi series of products useable by nearly anyone, Venture Networks intends to follow suit with the MVP developed. In the case of Ubiquiti, the UniFi SDN switches are centrally configured and managed, with the term SDN being used for marketing. The approach to online or central management of network devices is not new, with other companies such as Cambium Networks and Mimosa offering centralised, online control of their devices.

While no product specific to the WISP market and comparable to the MVP currently exists, Venture Networks believes it is only a matter of time before another manufacturer creates one. The results of the Grounded Theory study suggest that there is a market need for more straightforward to use, higher-performance routing and switching platforms for WISPs. This theory is also well backed by academic literature, which supports the idea that network operators often lack intricate technical expertise. In further support of this theory, the market trend for networked devices is low-effort, easy management, and centralised control. The 
combination of evidence illustrates why Venture Networks must see the feasibility study and commercialisation processes through to fruition, and in a timely manner.

\section{7 - Marketing}

Venture Networks believes that rather than spending money to market and promote the platform, a hands-on approach to developing customer interest will be taken. Venture Networks has observed the success other companies such as Ubiquiti Networks and Netonix have had with this approach. Rather than making eye-catching advertisements, both companies have their engineers interact directly with potential customers, typically over social media and on forums. Having engineers involved in product development readily available also acts as a form of damage control when a product is not working as intended, or something goes wrong.

Aside from providing customers with an approachable means to interact with engineers and offer feedback, Venture Networks will seek out WISPs struggling with network design and provide help. As a condition of helping, Venture Networks will require that the WISP uses the provided MVP, which will be provided on a free-of-charge basis until the network design is complete and operating well. The general concept is to build a strong customer following and enable customer-driven promotion of the MVP and Venture Networks. This approach will be crucial in finalising product development and discovering any unforeseen features that are required. However, to avert any avoidable implementation mistakes, this stage of customer development will be completed following the successful completion of the feasibility study.

The researcher also believes the MVP should not be marketed as an SDN platform, as the indepth interviews conducted showed WISPs are generally hesitant about the concept of 'SDN', with several participants believing it to be an industry buzzword with little real-world meaning. The MVP may also be sold under a different trading name, as Venture Networks is 
associated with the company's Internet services business. While an appropriate trading name has not been decided upon, several ideas have been brainstormed, and appropriate steps have been taken to register names that may be used in the future for selling the MVP.

\section{8 - Regional benefits}

As touched on earlier, the development of the MVP will enable two significant benefits to be realised. Better connectivity through the completion of the proposed feasibility study, which involves substantially increasing the reach of Venture Networks' Internet infrastructure across the Horowhenua district. Expanded coverage may improve the social wellbeing for residents living rurally who have limited Internet connectivity. Venture Networks' Internet service is affordable and provides enough throughput to customers for present and future demands, enabling users to surf the web, download games, or stream high-definition content as they desire. The expanded network coverage would also mean Venture Networks will be able to provide the two Marae mentioned earlier with high-speed connectivity, free of charge. Venture Networks believes in social responsibility and recognises the social good that will come out of supporting the local community.

Secondly, the development and real-world testing of the MVP will enable Venture Networks to help remote and rural communities on a much larger, potentially global scale. For remote and rural broadband access to be ubiquitous, changes across various domains must be made. The challenges involved with building remote and rural broadband infrastructure means that social, political, and technical changes are needed to encourage development. Venture Networks is contributing a technical solution that solves a combination of social and technical challenges. For those in areas that want better Internet connectivity but lack the skills required to build it, the MVP is a step forward towards making the Internet infrastructure development process more accessible. 
Finally, if Venture Networks completes the proposed feasibility study, it will enable the creation of additional jobs in the Manawatu-Whanganui region. As mentioned, the manufacturing of the MVP and subsequent developments may not be financially feasible to carry out in the Horowhenua. However, the research and design of systems can be kept within the Horowhenua district. Aside from job creation through research and development roles, an expanded network means that additional staff can be employed for customer installations, network maintenance, and other general operational duties.

\section{9 - Human resource requirements}

The development of the MVP has required extensive work from the researcher, and collaborative input from other staff at Venture Networks. At this stage, Venture Networks believes it is unnecessary to employ any additional staff to complete development of the MVP. However, upon starting a feasibility study of the MVP, Venture Networks will likely need additional temporary employees to help build the planned sites within the proposed timeframe. Following the successful completion of the feasibility study, Venture Networks will seek to employ additional research and design engineers, and other staff as necessary to see the MVP through to its full commercialisation potential.

\subsection{0 - Financial requirements and returns}

Capital is sought to complete the proposed feasibility study. Venture Networks believes that an appropriate source of funding for the feasibility study would be the Provincial Growth Fund (PGF). Under the PGF, a variety of regional projects can be funded. Infrastructure (tier 3) projects are one category of projects that the PGF can fund. Of infrastructure projects, the PGF states that they are particularly interested in digital projects that combine elements of: 
"deploying network infrastructure beyond existing broadband investments, to expand the reach and enhance the quality of digital connectivity available in the regions for lifting productivity" (MBIE, n.d.-a).

While the MVP itself is not an infrastructure project, conducting a feasibility study of its technical functionality enables Venture Networks to expand its rural Internet infrastructure, and potentially create jobs for the wider Manawatu-Whanganui region.

While there is commercialisation opportunity for the MVP, accurately projecting unit sales cannot yet be done with confidence. To better gauge potential future sales, a set of conservative forecasts have been drafted. The graphs are purely speculative and will be updated once actual sales of the unit take place. In practice, it is anticipated the sales volume will be much higher: 
The graphs above assume:

- A minimum of 25 units can be sold monthly.

- Unit sales are increasing by $5 \%$ monthly.

- 90 days' worth of inventory is kept on hand.

- The minimum order quantity from the contract manufacturer is 100 units.

- Units can be manufactured for approximately $\$ 60 \mathrm{NZD}$, including shipping costs and excluding GST.

- Units have a market value of approximately \$300 NZD, excluding GST.

- $10 \%$ of monthly revenue will be kept as a contingency against issuing refunds.

- Sales will begin in October 2019.

Accounts receivable:

- $25 \%$ of sales will be on credit terms.

- An average of 30 days is expected for credit repayments.

- An average of $0.05 \%$ of invoices will go unpaid.

Accounts payable:

- $50 \%$ of purchases will be on credit.

- An average of 30 days is expected for credit purchase repayments.

Taxes:

- Income tax is a flat rate of $28 \%$. 
If early sales of the MVP are above expectations, Venture Networks will reassess whether it needs to take on debt to support the future development and commercialisation of the

platform. 


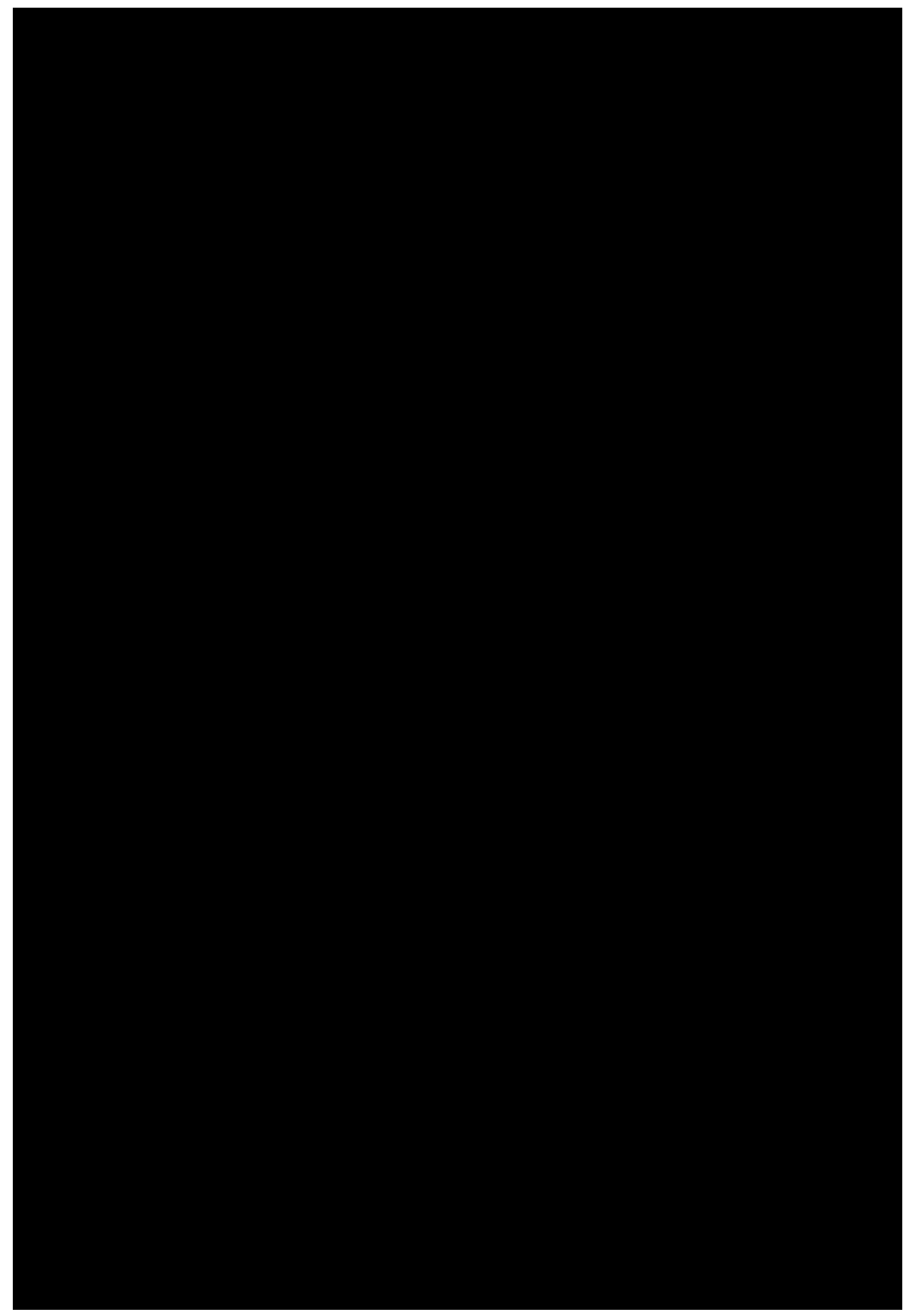




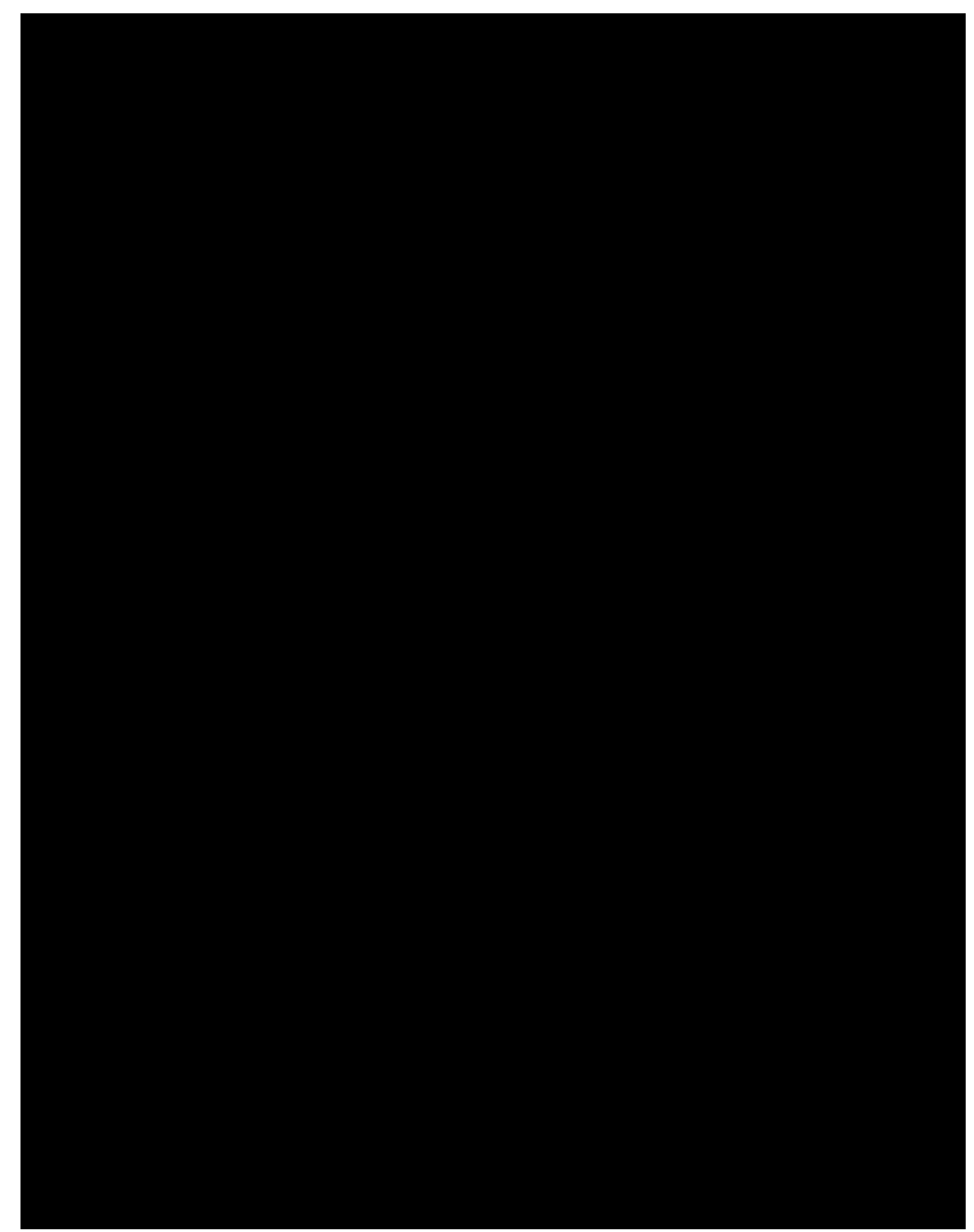

An overview of the minimum costs required to complete the feasibility study are shown in the table below. Staff wages are excluded, as Venture Networks does not believe it is appropriate to request funding for staff that are already accounted for. Wholesale Internet access is also excluded, as Venture Networks can manage the demand for traffic as necessary: 
Finally, the above table does not account for the cost of the MVP device that will be used on each network site for the feasibility study, which is approximately $\$ 60$ NZD per unit, excluding GST. Each site in the network expansion proposed for testing the feasibility of the MVP will enable over 100 rural users to connect to super-fast rural broadband, extending connectivity across the Horowhenua district far beyond existing government RBI1/RBI2 investments. Despite RBI connectivity options being available, and other WISPs operating in the wider region, Venture Networks believes the rural Horowhenua will benefit from a locally owned and operated network.

\subsection{1 - Business continuity planning}

In the case that the feasibility study shows significant limitations of the MVP, Venture Networks will be able to continue to service the additional areas of the Horowhenua as initially planned. Funding for the feasibility study is required for expanding the reach of Venture Networks' infrastructure across the Horowhenua, but not for the development of the MVP. The expanded network will provide Venture Networks with an ideal testbed for further development of the MVP while improving Internet connectivity to those living in the rural Horowhenua. If the MVP is deployed and does not meet satisfactory performance 
requirements, Venture Networks will replace MVP devices operating at network sites with commercially suitable alternatives.

In the case of the MVP not working as expected, the devices will be donated to the School of Engineering and Computer Science at Victoria University of Wellington. While an immediate use for the devices may not be found, the OpenWrt platform is extensible and could be used for any number of experiments, or to teach students about OpenFlow and other networking protocols. While not an ideal scenario, Venture Networks believes donating the equipment to be a socially and environmentally sensible decision.

\subsection{2 - Regulatory compliance}

For introducing the MVP into international markets, some key regulatory processes must be considered. For exporting to the European Union and the United States, Venture Networks must have the following certifications:

- RoHs approval (Restriction of Hazardous Substances) - all applicable products sold in the European Economic Area must pass RoHS compliance.

- Certified European (CE) - needed before the MVP can be sold in the European Economic Area.

- FCC Declaration of Conformity - confirms that the MVP does not emit unsafe levels of electromagnetic interference or accept interference from other devices. Needed for devices sold to the United States.

Venture Networks has worked with the contract manufacturer in China to have the low-cost OpenWrt platform used for the MVP to meet the required certification standards. The contract manufacturer operates a Quality Management System in conformity with ISO 9001:2008, and has successfully attained RoHS approval, CE approval, and FCC certification for the OpenWrt platform. The MVP should also comply with AS/NZS CISPR 22, the 
Australia/New Zealand standard required for information technology equipment. Venture Networks is in the process of seeking certification of the MVP under this standard.

\subsection{3 - Conclusion}

Despite some technical limitations of using an SDN-based MVP, Venture Networks believes that the use of SDN-technologies is a necessary trade-off to encourage low barrier to entry Internet infrastructure development in remote and rural communities. Alternatively, a significant amount of additional development would be required, and likely be outside the resource capabilities of a small company such as Venture Networks. For those living in remote and rural communities, the MVP does not need to spark a revolution. It merely needs to be user-friendly and enable potential WISP operators to build networks that are of acceptable quality without causing them unnecessary stress.

Venture Networks believes that the development of Grounded Theory shows that there is indeed an opportunity for such a product, and has provided the foundation of what technical requirements are necessary for the platform. Given the findings, Venture Networks believes the MVP has strong commercialisation potential. However, the true commercialisation potential of the platform will not be understood until the feasibility study has been completed, and Venture Networks brings the product to market. Of course, money is the best market validation tool available. Venture Networks has received positive feedback of the MVP so far, but if customers are genuinely convinced of the MVP's value, then it will be proven through sales. 


\section{Chapter 6 - Conclusion}

This research has identified through the Grounded Theory study that there is a market opportunity for more accessible to use routing/switching platforms for the remote and rural service provider, or typically WISP, market. The results of the Grounded Theory study are well supported by literature, confirming that WISP operators often struggle with the technical setup of their networks. Through open-ended, in-depth interviews, and the analysis of months of social media posts, the researcher has been able to identify where existing platforms fall short, and what is necessary to improve upon them.

Given the findings, the researcher is confident that the MVP can be positioned as a platform that anyone can use - not just technical experts and experienced network engineers. The Software-Defined Networking based MVP will combine a seamless user experience while incorporating useful, otherwise difficult to implement, technical functionalities. This should mean that WISP operators will no longer have concern that technical configurations have not been completed successfully. As a result, WISP operators will have less dependence on external help, such as consultants, and may experience better business outcomes. Ultimately, the easy-to-use nature of the MVP is progress towards the goal of ubiquitous remote and rural broadband access.

While not without challenges that will be explored throughout the proposed feasibility study, the MVP offers significant benefits to those looking to, or already running a WISP network. For large, well-resourced providers, the SDN solution may not be necessary. However, for those without technical skills, or even without a large team to help support ongoing configurations and troubleshooting, the MVP will offer friendly usability, and potentially cost savings through the reduced need for extra technical staff or external consultants. Just as Ubiquiti built an SDN-based solution for the enterprise market that has already proven itself to be successful, the SDN-based MVP will offer similar benefits to the WISP market. 
The MVP is the natural next step towards easier to build Internet access infrastructure in remote and rural communities. The growing WISP market has resulted in innovative radio usability, primarily thanks to Ubiquiti Networks, and later through a series of competitors such as Cambium Networks and Mimosa. In a sense, the existence of Ubiquiti itself confirms there is a market opportunity for more straightforward to use network hardware, especially for WISP network operators. Ubiquiti understood that their target market of remote and rural service providers is underserved and plagued by high-prices and slow product innovation. While Ubiquiti has disrupted radio usability, it has been identified there is still opportunity to improve the usability of other networking products typically used by WISPs.

The MVP renders the manual configuration of complex routing and switching solutions to be unnecessary. Using the MVP, WISPs will no longer require years of technical expertise in routing and switching, nor will they need to hire consultants when they lack otherwise necessary knowledge. Just as someone with basic computer literacy can configure a home router, the MVP enables someone with similar skills to configure and operate the routing and switching component of running a WISP. Subsequent additions to the MVP will likely further extend the platform to incorporate and help alleviate other market pains identified throughout the in-depth interviews. As the MVP is built on top of a modular platform, there are few limits as to what features could be added. Power management and monitoring, smarter spectrum utilisation (through the fine-grained control of connected radios), and performance analytics reporting are all possibilities that could be considered for future addition.

To address the market needs of New Zealand WISPs, the next logical addition to the MVP will be well-integrated power management. The typical approach to ensuring there is ample network capacity for peak demand is resource overprovisioning. In an environment where on-grid power is available, resource overprovisioning may waste energy during off-peak periods, where demand for throughput is at a minimum. In a WISP environment, overprovisioned radios without an adequate supply of off-grid energy will waste energy and 
could be detrimental to network reliability. As a result, WISPs such as those in New Zealand either need more affordable off-grid power systems, or network hardware that has improved power management.

In the case of the MVP, power management could be added to turn off radios during off-peak periods. As a result, WISPs could reduce the need for expensive power system upgrades, while offering greater network capacity during times of peak demand. The central SDN controller of the MVP could also be extended to coordinate power control across a generic WISP topology. Compared to other approaches, such as the manual creation of scripts to turn off network devices when certain conditions are met, a centralised approach would enable constraint-responsive changes to be made in real time. Additionally, implementing wellintegrated power control will not require an additional hardware platform, as the productionready iteration of the MVP will be PoE enabled.

Adding power control to the MVP will be considered following the successful completion of the proposed feasibility study. The addition of power-control may make the MVP attractive to those outside of the initial target market. Large WISPs typically have staff responsible for managing technical implementations, and the MVP may not be necessary. However, such large WISPs could still improve their financial outlooks through the reduced need for overprovisioned off-grid power systems. For Venture Networks, and other WISPs across New Zealand and potentially the world, a product that assists in effectively managing these constrained financial resources would be of great benefit.

Despite no similar platforms for the WISP market currently existing, the time to bring the MVP to market is now. If the proposed feasibility study and remaining commercialisation processes of bringing the MVP to market are delayed for too long, another competitor, such as Ubiquiti Networks, may develop a similar product. Access to potential funding sources such as the Provincial Growth Fund is also time sensitive, as the fund is already over a third of the way through its 3-year lifecycle. For the researcher and Venture Networks, time is of the 
essence. The global demand for remote and rural Internet access is increasing rapidly, as the dependence society has towards the Internet increases as an integral part of life. 


\section{References}

Allan, G. (2003). A critique of using grounded theory as a research method (Vol. 2).

BEBA. (2019). Key concept. Retrieved from http://www.beba-project.eu/the-project/keyconcept

Beck, K., Beedle, M., Bennekum, A. v., Cockburn, A., Cunningham, W., Fowler, M., . . . Thomas, D. (2001). Manifesto for Agile Software Development. Retrieved from https://agilemanifesto.org/

Ben-David, Y. (2015). Connecting the last billion. (Doctor of Philosophy PhD), University of California, Berkeley, University of California. Retrieved from http://digitalassets.lib.berkeley.edu/etd/ucb/text/BenDavid berkeley 0028E 15758.pd $\underline{\mathrm{f}}$

Blantz, E., \& Summer, M. (2011, 24-27 Oct. 2011). The Rural Broadband Initiative Toward a new model for broadband access in Haiti and beyond. Paper presented at the 2011 Technical Symposium at ITU Telecom World (ITU WT).

Charmaz, K. (2013). Constructing grounded theory (2nd ed.. ed.). London: SAGE.

Chesbrough, H. (2010). Business Model Innovation: Opportunities and Barriers. Long Range Planning, 43(2), 354-363. Retrieved from

http://www.sciencedirect.com/science/article/pii/S0024630109000569. doi:10.1016/j.lrp.2009.07.010

CIP. (2018). Crown Infrastructure Partners - RBI2/MBSF expansion announcement -

Questions and Answers. Retrieved from

https://www.beehive.govt.nz/sites/default/files/2018-12/RBI2MBSF\%20expansion\%20Q\%26A\%20-\%2018\%20Dec\%202018\%20FINAL.pdf

Commission, C. (2012). Vodafone pays hefty price for misleading consumers. Retrieved from http://www.comcom.govt.nz/the-commission/media-centre/mediareleases/2012/vodafone-pays-hefty-price-for-misleading-consumers/

Cooney, A. (2010). Choosing between Glaser and Strauss: an example: Adeline Cooney looks at the reasons for choosing either Glaserian or Straussian grounded theory when conducting research and why she made her choice in a recent study. (grounded theory)(Report). Nurse Researcher, 17(4), 18. doi:10.7748/nr2010.07.17.4.18.c7921

Corner, S. (2016). Rural Broadband Initiative phase one now complete, says Adams. Retrieved from https://www.computerworld.co.nz/article/604809/rural-broadbandinitiative-phase-one-now-complete-says-adams/

Coxhead, A. (2013). Improving the Security and Efficiency of Network Clients Using OpenFlow. (Bachelor of Computing and Mathematical Sciences with Honours Honours), University of Waikato, Hamilton. Retrieved from https://wand.net.nz/sites/default/files/520-adam-coxhead.pdf

Crothers, C., Smith, P., Urale, P. W. B., \& Bell, A. (2016). The Internet in New Zealand 2015. Retrieved from Auckland, New Zealand: https://workresearch.aut.ac.nz/ data/assets/pdf file/0003/71328/WIPNZ-Report060515.pdf 
Detti, A., Pisa, C., Salsano, S., \& Blefari-Melazzi, N. (2013). Wireless Mesh Software Defined Networks (wmSDN). In (pp. 89-95).

Discher, S. (2013). Burning Bridges - Routing Your

Bridged WISP Network With

MikroTik. Retrieved from https://mywisptraining.com/wpcontent/uploads/2013/01/BridgedToRouted.pdf

Eppler, M. J., Hoffmann, F., \& Bresciani, S. (2011). New Business Models Through Collaborative Idea Generation. 15(06), 1323-1341. Retrieved from https://www.worldscientific.com/doi/abs/10.1142/S1363919611003751. doi:10.1142/s1363919611003751

Glaser, B. G., \& Holton, J. (2004). Remodeling Grounded Theory. Forum : Qualitative Social Research, 5(2).

Glaser, B. G., \& Strauss, A. L. (1967). The discovery of grounded theory strategies for qualitative research Chicago: Aldine Publishing Company.

Golder, P. N., \& Tellis, G. J. (1993). Pioneer Advantage: Marketing Logic or Marketing Legend? Journal of Marketing Research, 30(2), 158-170. doi:10.2307/3172825

Goodman, E., \& Henry, P. (2010). Product methodologies: what they are and how to avoid pitfalls. Paper presented at the $\mathrm{PMI}^{\oplus}$ Global Congress 2010, Washington, DC. . https://www.pmi.org/learning/library/product-methodologies-software-developmentprograms-6529

GoWireless NZ. (n.d.). Wireless Basics. Retrieved from https://www.gowifi.co.nz/wirelessbasics.html

Hansen, M. (n.d.). NZ Wireless Map. Retrieved from https://wirelessmap.markhansen.co.nz/

Hasan, S., Barela, M. C., Johnson, M., Brewer, E., \& Heimerl, K. (2019). Scaling Community Cellular Networks with CommunityCellularManager. Paper presented the 16th USENIX Symposium on Networked Systems Design and Implementation (NSDI 19), Boston.

Hasan, S., Ben-David, Y., Bittman, M., \& Raghavan, B. (2015). The Challenges of Scaling WISPs. In (pp. 3-11).

Hasan, S., Ben-David, Y., Scott, C., Brewer, E., \& Shenker, S. (2013). Enhancing rural connectivity with software defined networks. Paper presented at the Proceedings of the 3rd ACM Symposium on Computing for Development, Bangalore, India.

Henderson, B. J. (2013). Radio Mobile: Radio Propagation and Radio Coverage Computer Simulation Program. Retrieved from http://www3.telus.net/hendersb/documents/Radio\%20Mobile.pdf

Hoelzle, U. (Producer). (2012). OpenFlow @ Google - Urs Hoelzle, Google. Open Networking Summit 2012. Retrieved from https://www.youtube.com/watch?v=VLHJUfgxEO4

Hutchison, A. J., Johnston, L. H., \& Breckon, J. D. (2010). Using QSR-NVivo to facilitate the development of a grounded theory project: an account of a worked example. International Journal of Social Research Methodology, 13(4), 283-302. doi:10.1080/13645570902996301 
IgniteNet. (2018). $60 \mathrm{GHz}$ MetroLinq. Retrieved from

https://www.ignitenet.com/technology/metroling/

International Telecommunication Union. (2017). The State of Broadband 2017.

Kromer, T. (2014). The Four Parts of a Minimum Viable Product. Retrieved from

https://grasshopperherder.com/the-four-parts-of-a-minimum-viable-product/

Luo, Z., \& Suh, C. (2011, 26-28 Jan. 2011). An improved shortest path bridging protocol for

Ethernet backbone network. Paper presented at the The International Conference on

Information Networking 2011 (ICOIN2011).

ManagementNext. (2012). The Agile Manifesto. ManagementNext.

Network operators with fewer than 4000 customers, (2013).

MBIE. (2017). Ultra-Fast Broadband, Rural Broadband Initiative Phase Two And Mobile Black Spot Fund - Q\&A. Retrieved from

https://www.crowninfrastructure.govt.nz/wp-content/uploads/2017/08/Questionsand-Answers.pdf

MBIE. (2018). Broadband and mobile programmes. Retrieved from

https://www.mbie.govt.nz/science-and-technology/it-communications-and-

broadband/fast-broadband/broadband-and-mobile-programmes/\#rural-broadbandinitiative

MBIE. (n.d.-a). Infrastructure Projects. Retrieved from https://www.growregions.govt.nz/getfunding/projects-we-can-fund/infrastructure-projects/

MBIE. (n.d.-b). Projects we can fund. Retrieved from https://www.growregions.govt.nz/getfunding/projects-we-can-fund/

Netonix. (2014). Our Story. Retrieved from https://www.netonix.com/about-netonix/

NSRC. (2016). About. Retrieved from https://nsrc.org/about

Nungu, A., Olsson, R., \& Pehrson, B. (2012). Design and Implementation of an Inclusive

Ubiquitous Access. An International Journal, 67(1), 79-94. doi:10.1007/s11277-012-

0801-5

NZNOG (Producer). (2019). NZNOG 2019 - Day 2 - Session 4. Retrieved from https://www.youtube.com/watch?v=7p1SH8-6KtI\&feature=youtu.be\&t=3526

Olimex. (2017). SMT-PRODUCTION. Retrieved from https://www.olimex.com/wiki/SMTPRODUCTION

Osterwalder, A. (2004). The Business Model Ontology A Proposition In A Design Science Approach. (PhD), University of Lausanne,

Osterwalder, A. (2010). Business model generation a handbook for visionaries, game changers, and challengers. In Y. Pigneur \& T. Clark (Eds.). Hoboken, NJ: Wiley.

Porter, M. E. (1998). Competitive strategy: techniques for analyzing industries and competitors (1st Free Press ed.. ed.). New York: Free Press.

Pötsch, T., Yousaf, S., Raghavan, B., \& Chen, J. (2018). Zyxt: A Network Planning Tool for Rural Wireless ISPs.

Raza, S. (2017, June 23). Ubiquiti Networks Inc (UBNT), A Case Study In Business Model Disruption. Retrieved from https://www.valuewalk.com/2017/06/ubiquiti-networksinc-ubnt-case-study-business-model-disruption/ 
Ries, E. (2011). The lean startup : how today's entrepreneurs use continuous innovation to create radically successful businesses / Eric Ries (1st ed.. ed.). New York: Crown Business.

RSM. (2016). Licence Summary: 134726. Retrieved from

https://www.rsm.govt.nz/rrf/licence/number/208889

RSM. (2017). Radiocommunications Regulations (General User Radio Licence for Short Range Devices) Notice 2017 No. 2. Retrieved from https://gazette.govt.nz/notice/id/2017-go4089

SamKnows. (2018). Measuring Broadband New Zealand: Initial findings report, 2018. Retrieved from

https://measuringbroadbandnewzealand.com/static/MBNZ\%20\%E2\%80\%93\%20Initi al\%20findings\%20report\%20\%E2\%80\%93\%2021\%20December\%202018.pdf

Singh, H., Prasad, R., \& Bonev, B. (2018). The Studies of Millimeter Waves at $60 \mathrm{GHz}$ in Outdoor Environments for IMT Applications: A State of Art. Wireless Personal Communications, 100(2), 463-474. Retrieved from https://doi.org/10.1007/s11277017-5090-6. doi:10.1007/s11277-017-5090-6

Sonba, A., \& Abdalkreim, H. (2014). Performance Comparison Of the state of the art Openflow Controllers. (Master's of Computer Network Engineering Master's), Halmstad University Halmstad. Retrieved from http://hh.divaportal.org/smash/get/diva2:783679/FULLTEXT02.pdf

Stampfl, G. (2016). The process of business model innovation: an empirical exploration. In (1st ed. 2016.. ed.). Wiesbaden, Germany: Springer Gabler.

Steenbergen, R. A. (2010). MPLS for Dummies. Retrieved from https://www.nanog.org/meetings/nanog49/presentations/Sunday/mpls-nanog49.pdf

Surana, S., Patra, R., Nedevschi, S., Ramos, M., Subramanian, L., Ben-David, Y., \& Brewer, E. (2008). Beyond Pilots: Keeping Rural Wireless Networks Alive.

Techatassanasoontorn, A., Andrade, A. D., Hedges, M., \& Karimikia, H. (2018). World Internet Project: New Zealand Internet in New Zealand in 2017. Retrieved from Auckland, New Zealand: https://workresearch.aut.ac.nz/ data/assets/pdf file/0009/174915/CoreReport Oct15 release.pdf

Timonen, V., Foley, G., \& Conlon, C. (2018). Challenges When Using Grounded Theory: A Pragmatic Introduction to Doing GT Research. International Journal of Qualitative Methods, 17(1), 160940691875808. doi:10.1177/1609406918758086

Toury, H. (2019). Ultra-fast broadband (UFB - UFB2/2+) roll out. Retrieved from https://sp.chorus.co.nz/news/ultra-fast-broadband-ufb-ufb22-roll-out

Ubiquiti Networks. (2017). Investor Presentation [Press release]. Retrieved from http://ir.ubnt.com/sites/default/files/2017-11/ubnt-investor-presentation-11-0917.pdf

Ubiquiti Networks. (n.d.). UniFi - USW: Configuring Spanning Tree Protocol. Retrieved from https://help.ubnt.com/hc/en-us/articles/360006836773-UniFi-USWConfiguring-Spanning-Tree-Protocol\#3 
University of Leicester. (n.d.). Appendix - Research Theory. Retrieved from https://www.le.ac.uk/oerresources/lill/fdmvco/module9/page 11.htm

Varma, T. (2015). Agile Product Development: How to Design Innovative Products That Create Customer Value. In (1st edition. ed.): Apress.

Willig, C. (2013). Grounded theory methodology. In Introducing Qualitative Research in Psychology Third Edition (pp. 69-82): Open University Press.

Zorah, S. (2016, March 31). How to choose the right DevOps tools. Retrieved from https://www.atlassian.com/blog/devops/how-to-choose-devops-tools 


\section{Appendices}

Appendix 1 - Information Sheet for Interview Participants

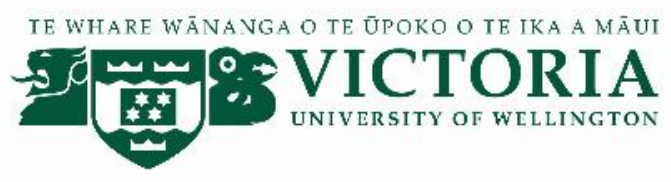

\section{Broadband for Remote and Rural Communities \\ A project in innovative network technologies and their commercialisation potential}

\section{INFORMATION SHEET FOR INTERVIEW PARTICIPANTS}

You are invited to take part in this research. Please read this information before deciding whether or not to take part. If you decide to participate, thank you. If you decide not to participate, thank you for considering this request.

\section{Who am I?}

My name is Duncan Cameron, and I am a master's student in the Master of Innovation and Commercialisation programme at Victoria University of Wellington. This research project is work towards my thesis.

\section{What is the aim of the project?}

This project is designed to develop a thorough understanding of rural Internet users and the geographic digital divide. Throughout this project, the potential for developing innovative low-cost Internet network infrastructure and the subsequent commercialisation potential will be analysed. The overall aim of this is to make rural Internet faster and more accessible (cheaper). This research has been approved by the Victoria University of Wellington Human Ethics Committee; reference number 26140. 


\section{How can you help?}

You have been invited to participate because you are situated rurally and/or operate a WISP. If you agree to take part, I will interview you at a suitable location, such as your home, or a local café. Alternatively, interviews can take place over the video call or the phone. If you are not comfortable, I can send you a set of interview questions to complete over email. I will ask you questions about rural Internet connectivity. The interview will take approximately thirty minutes. You can choose to not answer any question or stop the interview at any time, without giving a reason. You can withdraw from the study by contacting me at any time within four weeks of the interview. If you withdraw, the information you provided will be destroyed or returned to you.

\section{What will happen to the information you give?}

This research is confidential. This means that the researcher named below will be aware of your identity, but the research data will be combined, and your identity will not be revealed in any reports, presentations, or public documentation. However, you should be aware that in small projects your identity might be obvious to others in your community. You will not be named in the final report, but your organisation will be named (provided you have the authority to agree to this on behalf of the organisation).

Only my supervisors and I will read the notes or transcript of the interview. The interview transcripts, summaries and any recordings will be kept securely and destroyed on 01/06/2019.

\section{What will the project produce?}

The information from my research will be used in my master's thesis and/or academic publications and conferences.

\section{If you accept this invitation, what are your rights as a research participant?}

You do not have to accept this invitation if you don't want to. If you do decide to participate, you have the right to: 
- $\quad$ choose not to answer any question;

- $\quad$ ask for the recorder to be turned off at any time during the interview;

- $\quad$ withdraw from the study within four weeks of the interview;

- $\quad$ ask any questions about the study at any time;

- $\quad$ receive a copy of your interview recording;

- $\quad$ receive a copy of your interview transcript;

- $\quad$ read over and comment on a written summary of your interview;

- $\quad$ be able to read any reports of this research by emailing the researcher to request a copy.

If you have any questions or problems, who can you contact?

If you have any questions, either now or in the future, please feel free to contact either:

\section{Student:}

Name: Duncan Cameron

University email address:

camerodunc1@myvuw.ac.nz

\section{Supervisor:}

Name: Dr Alvin Valera

Role: Senior Lecturer

School: School of Engineering and

Computer Science

Phone: 0446351039

alvin.valera@ecs.vuw.ac.nz

\section{Human Ethics Committee information}

If you have any concerns about the ethical conduct of the research you may contact the Victoria University HEC Convenor: Dr Judith Loveridge. Email hec@vuw.ac.nz or telephone +64-4-463 6028 . 


\section{Appendix 2 - Interview Questions}

Note: Interview questions were adapted based on where the interview participant was from, e.g., New Zealand would be substituted for the USA. Questions were open-ended and designed to encourage discussion of other topics too.

\section{Survey Questions for Service Providers (original):}

1. What motivated you to work at/start a rural broadband network?

2. Do you believe technical hardware within a rural broadband network needs to be simplified to encourage wide-spread network deployment?

3. What frustrates you most during your day-to-day business operations?

4. If you were given an open budget, what would you add to/upgrade on your network?

5. Do you believe the network infrastructure you run is well utilised (e.g., backup/redundant links are often utilised and have traffic load-balanced over them)?

6. If you were able to eliminate any single frustration from your rural broadband network, what would it be?

7. Do you find that network equipment is suited to the New Zealand natural environment (i.e., in terms of weatherproofing/ruggedness)?

8. Do you find new/prospective customers are sceptical of wireless technology and reluctant to use it?

9. Do you believe any legislative changes need to occur to improve rural broadband development through smaller providers (such as spectrum allocations, changes to allocated power budgets, land access/easements, etc)?

10. Have you explored the possibility of using Software-Defined Networking on your rural broadband network?

11. Can you identify any recurring network expenses you incur that could be reduced through automation and/or technical development?

12. Can you think of any commonly recurring 'roadblocks' that are experienced by rural broadband providers (new and mature) New Zealand wide? 


\section{Survey Questions for Service Providers (modified):}

1. What motivated you to work at/start a rural broadband network?

2. Are you responsible for network design at your company?

3. Do you have a standardised methodology to your network planning?

4. What data do you currently gather for monitoring/network reliability assurance?

5. Do you have a unified interface for viewing this monitoring data?

6. Do you use an asset management system for your routers/radios/other high-value gear?

7. Do you think your company is spending money on avoidable costs, e.g., re-work being done due to poor site planning, etc.

8. If avoidable costs are occurring, are these of major concern, or are there more pressing financial pressures?

9. What motivations do you have to reduce such expenses?

10. Do you believe technical hardware within a rural broadband network needs to be simplified to encourage wide-spread network deployment?

11. What frustrates you most during your day-to-day business operations?

12. If you were given an open budget, what would you add to/upgrade on your network?

13. Do you believe the network infrastructure you run is well utilised (e.g., backup/redundant links are often utilised and have traffic load-balanced over them)?

14. If you were able to eliminate any single frustration from your rural broadband network, what would it be?

15. Do you find that network equipment is suited to the New Zealand natural environment (i.e., in terms of weatherproofing/ruggedness)?

16. Do you find new/prospective customers are sceptical of wireless technology and reluctant to use it?

17. Do you believe any legislative changes need to occur to improve rural broadband development through smaller providers (such as spectrum allocations, changes to allocated power budgets, land access/easements, etc)? 
18. Have you explored the possibility of using Software-Defined Networking on your rural broadband network?

19. Can you identify any recurring network expenses you incur that could be reduced through automation and/or technical development?

20. Can you think of any commonly recurring 'roadblocks' that are experienced by rural broadband providers (new and mature) New Zealand wide?

21. Are there any additional comments you would like to make that might benefit this research?

22. Would you be willing to answer additional research questions in the future?

\section{Further Questions:}

1. What benefit would your company experience from being able to better utilise available spectrum?

2. Is network automation (e.g., reducing configuration times, human interaction with network devices, etc) important to you and your company?

3. What cost reduction or service improvement do you feel would be necessary for such a device/network appliance to be viable for use on your network?

4. If you had a blank cheque and a team of experienced engineers, what functionality would you add to routers and or other network devices running on your network?

5. Thinking about your network as it is now, what areas do you think could be improved?

6. Are there any other comments you would like to add? 